\title{
THE ELEMENTAL COMPOSITION OF HUMAN TEETH WITH EMPHASIS ON TRACE CONSTITUENTS A REVIEW
}

\author{
William H. Sachs
}

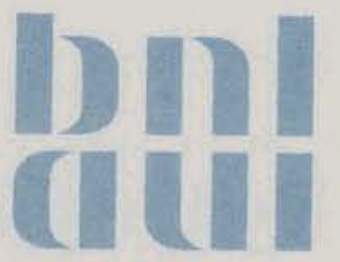

APRIL 1978

BROOKHAVEN NATIONAL LABORATORY ASSOCIATED UNIVERSITIES, INC. UNDER CONTRACT NO. EY-76-C-02-0016 WITH THE UNITED STATES DEPARTMENT OF ENERGY 


\section{DISCLAIMER}

This report was prepared as an account of work sponsored by an agency of the United States Government. Neither the United States Government nor any agency Thereof, nor any of their employees, makes any warranty, express or implied, or assumes any legal liability or responsibility for the accuracy, completeness, or usefulness of any information, apparatus, product, or process disclosed, or represents that its use would not infringe privately owned rights. Reference herein to any specific commercial product, process, or service by trade name, trademark, manufacturer, or otherwise does not necessarily constitute or imply its endorsement, recommendation, or favoring by the United States Government or any agency thereof. The views and opinions of authors expressed herein do not necessarily state or reflect those of the United States Government or any agency thereof. 


\section{DISCLAIMER}

Portions of this document may be illegible in electronic image products. Images are produced from the best available original document. 
BNL 50846

UC-48

(Biology and Medicine - TID-4500)

\title{
THE ELEMENTAL COMPOSITION OF HUMAN TEETH WITH EMPHASIS ON TRACE CONSTITUENTS A REVIEW
}

\author{
William H. Sachs
}

APRIL 1978

$$
\begin{aligned}
& \text { This report was prepared as an account of work } \\
& \text { sponsored by the United States Government. Neither the } \\
& \text { United States nor the United States Department of } \\
& \text { Energy, nor any of their employees, nor any of their } \\
& \text { contractors, subcontractors, or their employees, makes } \\
& \text { any warranty, express or implied, of assumes any legal } \\
& \text { liability or responsibility for the accuracy, completeness } \\
& \text { or usefulness of any information, apparatus, product or } \\
& \text { process disclosed, or represents that its use would not } \\
& \text { infringe privalely owned rights. }
\end{aligned}
$$

Research supported in part under contract no. EY-76-C-02-0016 with the U.S. Department of Energy at Brookhaven National Laboratory and by the U.S. Environmental Protection Agency under Agreement \#EPA-IAG-D5-E681-A0 (sub-agreement \#77EA0) with the U.S. Department of Energy 


\section{NOTICE}

This report was prepared as an account of work sponsored by the United States Government. Neither the United States nor the United States Department of Energy (DOE), nor any of their employees, nor any of their contractors, subcontractors, or their employees, makes any warranty, express or implied, or assumes any legal liability or responsibility for the accuracy, completeness or usefulness of any information, apparalus, product or process discloscd, or represents that its use would not infringe privately owned rights.

Printed in the United States of America

Available from

National Technical Information Service U.S. Department of Commerce

5285 Port Royal Road

Springfield, VA 22161

Price: Printed Copy $\$ 7.25$; Microfiche $\$ 3.00$ 
Acknowledgements

It is a pleasure to acknowledge the assistance of L. G. Stang, Jr., in the preparation of this manuscript. In addition. to considerable editorial help, he also conducted portions of the literature search which form the basis of this paper. His interest has been material in bringing this review to fruition. 
This paper is an attempt to evaluate critically the diverse literature on the inorganic composition of human dental hard tissues. Analytical procedures used in preparing dental samples for chemical analysis are examined with emphasis on sources of sample contamination. An extensive tabulation of compositional data is provided and evaluated topically. Areas covered include the geographical variability of these data, and the influence on them of the external variables, donor age and sex. Variation in composition within individual dental specimens, within and between mouths, and between different types of teeth are also discussed. Where possible, the simple review format is augmented by critical comment and by our own analysis of the data. 
Introduction. . . . . . . . . . . . . . . . . . . . . . 1

Chapter 1. . . . . . . . . . . . . . . . . . . . . . . 3

1.1 Analysis . . . . . . . . . . . . . . . . . . 3

1.2 Sample preparation . . . . . . . . . . . . . . . . 4

1.3 Sampling Error . . . . . . . . . . . . . . . . . 18

Chapter 2 . . . . . . . . . . . . . . . . . . . . . . 22

2.1 Table Format . . . . . . . . . . . . . . . . 23

2.2 Statistical Analysis . . . . . . . . . . . . . . 59

2.3 Geographic Variation . . . . . . . . . . . . . . . 68

2.4 Age . . . . . . . . . . . . . . . . . . . . 83

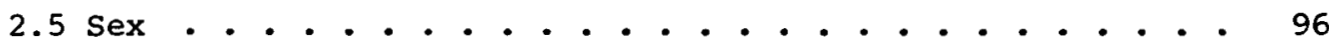

2.6 Distribution : . . . . . . . . . . . . . . . . . . 99

2.7 Tooth Type/Intermouth Variation. . . . . . . . . . . 108

2.8 Miscellaneous . . . . . . . . . . . . . . . . 115

2.9 summary . . . . . . . . . . . . . . . . . . 118

References . . . . . . . . . . . . . . . . . . . 120 


\section{Introduction}

The literature on the inorganic 'composition of teeth' is extensive. The review by Rowles: (1967) on dentine, citing 293 references through 1966; by Brudevold and Soremark (1967) on enamel, citing 171 references through 1965; by zipkin (1970) on bones and teeth, citing 107 references through 1967; and by Nixon (1969) on trace element levels in enamel, dentine, and plaque, citing 35 references through 1967, provide broad coverage of this field through the mid-sixties. Since then, over 89 papers have been published dealing with this topic; activity due in part to interest in teeth as possible indicators of environmental levels of trace metals of anthropogenic origin.

Literature covered by the current review is based on a search of Chemical Abstracts, 1917 through 1975. Early studies, pre-dating 1940, are referenced primarily for historical interest. Emphasis is on the microconstituents of human teeth, those present at concentrations less than a few tenths of a percent by weight. Within this category of data, we have been primarily concerned with the radiochemically stable nuclides. The important relationship between caries and trace elements is covered only insofar as carious teeth exhibit properties with respect to trace element composition that differ from normal teeth. Having made these disclaimers, we note that an attempt has been made to cover the literature exhaustively; although some important results have undoubtedly been overlooked. It is our hope, however, that sufficient material has been included in this review to facilitate further recovery of data by interested individuals.

This report will be structured as follows: In Chapter 1, analytical techniques that have been employed in this field will be briefly presented; 
discussion will center on problems associated with preparation of specimens for analysis: Chapter 2 will be devoted to topical coverage of data on the inorganic composition of teeth. An element-by-element tabulation of concentration data is provided, our statistical analysis of selected data explained, and evidence concerning several major factors thought to influence dental composition evaluated. These include provenance, age, sex, distribution, and tooth type/intermouth variation. 


\section{$\underline{\underline{1.1}} \underline{\underline{\text { Analysis }}}$}

Techniques that have been employed for the measurement of the inorganic content of teeth are of wide ranging characteristics. The method or methods of choice are often dictated by resources available to the investigator; ideally, however, the methodology will be determined by the overall requirements of the investigation. Studies whose focus is the measurement of a single constituent of teeth enjoy a freedom of choice with respect to maximum sensitivity, low detection limit, and minimum interference not available to studies involving multielement analyses. For the latter, where the goal is the simultaneous measurement of as many elements as possible, sensitivities and detection limits will not be uniformly good for all elements.' Use of more than one technique may in some cases be necessary.

Instrumental methods for trace analysis have been extensively reviewed. References include Dulka and Risby (1976) [a variety of methods for the ultratrace analysis of metals in environmental and biological systems], Snooks (1972) [emission spectroscopy], Burrell (1972) [flame spectrophotometry], Weiss (1972) [visible spectrophotometry], Addink (1967) [optical and X-ray spectroscopy], West (1967) [molecular absorption and fluorescence, and atomic absorption and fluorescence spectroscopy], Smales (1967) [nuclear activation and tracer analysis], Kelly (1972) [neutron activation analysis], Laitinen (1967) [electrochemical methods], and Ahearn (1967) [spark source mass spectrometry]. Additional and more recent coverage of specific aspects of sampling, sample handling, and analysis of materials for trace constituents has been published recently [Proceedings of the 7th Materials Research Symposium, 1974. Printed 1976]. 
Nixon (1969), has reviewed several instrumental techniques for the analysis of. hard dental tissues, including spark source mass spectroscopy, activation analysis, emission flame spectrophotometry, and atomic absorption spectrophotometry. Mention is also made of standard wet chemical analysis. Since Nixon's review, several new techniques have been used in analyses of dental tissues, including X-ray fluorescence spectroscopy [Calonius et a1 (1965) and Kubota et al (1974)], anodic stripping voltammetry [de 1a Burde et al (1975); Needleman et al (1973, 1974a,b); and Shapiro et al (1972, 1973, 1975)], electron microprobe analysis [Bax et al (1969); Besic et al (1969); Carroll et al (1972); Kurosaki et al (1973); and We1 et al (1974)], and proton induced $\mathrm{X}$-ray emission spectroscopy [AhIberg et al (1976)].

of the techniques that have been employed in dental analyses, neutron activation, X-ray fluorescence spectroscopy, emission spectroscopy, protoninduced X-ray emission spectroscopy, and spark source mass spectrometry are capable of simultaneous multielement characterization. Each method is unique in terms of the advantages it offers and also, the problems that plague it. Nevertheless, these methods provide a maximum of information on the inorganic content of dental tissues with a minimum of effort. Table I lists the elements that have been measured or detected in human dental samples by the various techniques referred to above.

\section{$\underline{\underline{1.2}}$ Samp1e preparation}

Virtually every step in the preparation of a sample for analysis incorporates a potential for contamination. Where components of interest are present in the sample at trace levels, contamination is a serious problem requiring scrupulous attention to detail. Robertson (1972) has written a particularly sobering review of contamination problems in trace element analysis and ultrapurification, and has made suggestions for minimizing their effects. The 
Table 1. Analytical Procedures in the Trace

Analysis of Dental Specimens

Procedure

Atomic Absorption
Spectrophotometry

Cathode-Luminescence

Charged-Particle

Activation Analysis

Colorimetry (UV/Visible spectrophotometry)

Electron Microprobe Analysis

Emission Spectroscopy

Neutron Activation Analysis a

Polarography

Spark Source Mass Spectroscopy ${ }^{b}$

Voltammetry

Wet Chemical Analysis

(Titrimetric,

gravimetric, etc.)

$X$-Ray Emission Analysis, Proton Induced

$\mathrm{x}$-Ray Induced

\section{Elements Detected/Quantified}

$\mathrm{Ag}, \mathrm{Al}, \mathrm{Ca}, \mathrm{Cd}, \mathrm{Cu}, \mathrm{F}, \mathrm{I}, \mathrm{K}, \mathrm{Mn}, \mathrm{Pb}, \mathrm{Se}, \mathrm{Sn}, \mathrm{Sr}, \mathrm{Zn}$

$\mathrm{Bi}$

$\mathrm{C}, \mathrm{Pb}$

$\mathrm{Cu}, \mathrm{F}, \mathrm{P}, \mathrm{S}, \mathrm{Se}, \mathrm{Zn}, \mathrm{Zr}$

$\mathrm{Ca}, \mathrm{Cl}, \mathrm{F}, \mathrm{Mg}, \mathrm{Na}, \mathrm{P}, \mathrm{Pb}, \mathrm{Sn}, \mathrm{Zn}$

$\mathrm{Ag}, \mathrm{Al}, \mathrm{Au}, \mathrm{B}, \mathrm{Ba}, \mathrm{Be}, \mathrm{Bi}, \mathrm{C}, \mathrm{Ca}, \mathrm{Cl}, \mathrm{Co}, \mathrm{Cr}, \mathrm{Cu}$, $\mathrm{F}, \mathrm{Fe}, \mathrm{Hg}, \mathrm{K}, \mathrm{La}, \mathrm{Li}, \mathrm{Mg}, \mathrm{Mb}, \mathrm{Mo}, \mathrm{Na}, \mathrm{Ni}, \mathrm{P}, \mathrm{Pb}$, Pd, Pt, Si, Sn, Sr, Ti, V, Zn, Zr

$\mathrm{Ag}, \mathrm{Al}, \mathrm{As}, \mathrm{Au}, \mathrm{Ba}, \mathrm{Br}, \mathrm{Ca}, \mathrm{Cd}, \mathrm{Cl}, \mathrm{Co}, \mathrm{Cr}, \mathrm{Cu}, \mathrm{Fe}$, $\mathrm{Hg}, \mathrm{La}, \mathrm{Mg}, \mathrm{Mn}, \mathrm{Mo}, \mathrm{Na}, \mathrm{P}, \mathrm{Pb}, \mathrm{Pt}, \mathrm{Rb}, \mathrm{Sb}, \mathrm{Se}, \mathrm{Sm}, \mathrm{Sn}$, $\mathrm{Sr}, \mathrm{U}, \mathrm{V}, \mathrm{W}, \mathrm{Zn}$

$\mathrm{Cu}, \mathrm{Zn}$

$\mathrm{Ag}, \mathrm{Al}, \mathrm{As}, \mathrm{Au}, \mathrm{B}, \mathrm{Ba}, \mathrm{Be}, \mathrm{Bi}, \mathrm{Br}, \mathrm{C}, \mathrm{Ca}, \mathrm{Cd}, \mathrm{Ce}$, $\mathrm{Cl}, \mathrm{Co}, \mathrm{Cr}, \mathrm{Cs}, \mathrm{Cu}, \mathrm{F}, \mathrm{Fe}, \mathrm{Ga}, \mathrm{Ge}, \mathrm{H}, \mathrm{I}, \mathrm{In}, \mathrm{K}, \mathrm{La}$, $\mathrm{Li}, \mathrm{Mg}, \mathrm{Mn}, \mathrm{Mo}, \mathrm{N}, \mathrm{Na}, \mathrm{Nb}, \mathrm{Nd}, \mathrm{Ni}, \mathrm{O}, \mathrm{P}, \mathrm{Pb}, \mathrm{Pd}, \mathrm{Pr}$, $\mathrm{Pt}, \mathrm{Rb}, \mathrm{Ru}, \mathrm{S}, \mathrm{Sb}, \mathrm{Sc}, \mathrm{Se}, \mathrm{Si}, \mathrm{Sn}, \mathrm{Sr}, \mathrm{Ta}, \mathrm{Ti}, \mathrm{V}$, $\mathrm{Y}, \mathrm{Zn}, \mathrm{Zr}$

$\mathrm{Pb}$

$\mathrm{Ca}, \mathrm{F}, \mathrm{P}, \mathrm{Mg}, \mathrm{Ni}, \mathrm{Se}, \mathrm{Zn}$

$\mathrm{Br}, \mathrm{Ca}, \mathrm{Cl}, \mathrm{Cu}, \mathrm{Fe}, \mathrm{K}, \mathrm{Mn}, \mathrm{Ni}, \mathrm{P}, \mathrm{Pb}, \mathrm{S}, \mathrm{Sr}, \mathrm{Zn}$

$\mathrm{Ag}, \mathrm{Br}, \mathrm{Ca}, \mathrm{Cl}, \mathrm{Co}, \mathrm{Cr}, \mathrm{Cu}, \mathrm{Fe}, \mathrm{K}, \mathrm{Mn}, \mathrm{Ni}, \mathrm{P}, \mathrm{Pb}, \mathrm{Pd}$, $\mathrm{Sb}, \mathrm{Sn}, \mathrm{Sr}, \mathrm{Zn}, \mathrm{Zr}$

(a) Specifically sought, but not detected: Tb, Yb [Steinnes et al (1974)]

(b) With the exception of the rare gases, all 90 naturally occurring elements have been sought with this technique. Those not listed here have not been detected. (c) [Hardwick et al (1967); Losee et al (1974c)].

Presence possibly due to sample contamination [Hardwick et al (1967)]. 
following discussion of contamination problems in the analysis of dental tissues is patterned after Robertson's outline.

Physical manipulation :

A sample is always subject to physical manipulation prior to analysis. For dental hard tissues, this involves one or more of the following: (i) Extraction of the specimen from the donor; (ii) cleaning the specimen of adhering debris and foreign matter; (iii) removal from the specimen of the sample or samples to be analyzed; (iv) desiccation of the sample; and, (v) grinding operations.

\section{(i) Extraction of the specimen}

Contamination by $\mathrm{Fe}, \mathrm{Mn}$, $\mathrm{Cr}$, etc., of dental specimens extracted with steel forceps and surgical tools has not to our knowledge been evaluated, although in studies by Lundberg and coworkers (1965 a, b) precautions were taken to ensure that only polyethylene instruments were in contact with impacted bicuspids during surgical removal. Söremark and coworkers (1962a,b, 1964a,b) imply similar. precautions in the removal of erupted teeth, stating that (enamel and dentine) samples were at no time in contact with other than quartz and/or polythene prior to analysis, and Nixon et a1 (1967c) report using polythene protected forceps for specimen removal.

Contamination of at least the surface of the specimen by elements present in the extracting instruments is probably real. Its importance, however, may be restricted to studies focusing on the trace element content of the surface layers of enamel. Minczewski (1967), in a review of preconcentration techniques in trace analysis, pictures autoradiographs of aluminum blocks (a fairly soft metal) cut with steel and tungsten carbide turning tools which show contamination of the aluminum by the metals of the turning tools. An investigator relying on outside sources for specimens has little control over contamination resulting from methods used to extract teeth. 
Studies employing surface analysis techniques, for example, activation of the specimen by beams of accelerated ${ }^{3} \mathrm{He}^{+}$followed by autoradiography, should prove valuable in evaluating this source of contamination.

(ii) Cleaning the specimen of adhering debris and foreign matter

Conditions employed for cleaning dental specimens are rarely stated explicitly and their potential for contamination remains unevaluated. Brudevold et al $(1955,1956 \mathrm{a}-\mathrm{c})$ in preparing enamel samples for $\mathrm{Cu}, \mathrm{F}, \mathrm{Pb}$, and $\mathrm{Sn}$ determinations report using gentle brushing with pumice and distilled water. Little and Steadman (1966) scaled their specimens with hand instruments (stee1?) and, following further manipulation, cleansed them with a revolving stiff-bristle brush and distilled water. Enamel samples were later analyzed for $\mathrm{Ag}, \mathrm{Al}$, $\mathrm{Cr}, \mathrm{Cu}, \mathrm{F}, \mathrm{Fe}, \mathrm{Mn}, \mathrm{Pb}, \mathrm{Si}, \mathrm{Sn}, \mathrm{Sr}$, and $\mathrm{Zn}$. Needleman et al (1972) report cleaning specimens in detergent solution prior to $\mathrm{Pb}$ analysis, while Lockeretz (1975) ultrasonically cleaned specimens in detergent solution prior to $\mathrm{Pb}$. analysis. Retief et al (1971a, b, 1974) used polyethylene covered scalers for removal of debris and calculus, and employed a brush and pumice followed by washing in deionized water for final cleaning before analyses involving $\mathrm{Ag}$, $\mathrm{A} 1, \mathrm{Au}, \mathrm{Ba}, \mathrm{Br}, \mathrm{Ca}, \mathrm{Cl}, \mathrm{Co}, \mathrm{Cr}, \mathrm{Fe}, \mathrm{Mg}, \mathrm{Mn}, \mathrm{Na}, \mathrm{Sb}, \mathrm{Sr}$, and $\mathrm{Zn}$, while Langmyhr et al (1974, 1975), prior to analysis for $\mathrm{Ag}, \mathrm{Cd}, \mathrm{Mn}, \mathrm{Pb}$, and $\mathrm{Zn}$, scaled specimens with plastic instruments followed by brushing. Losee et al (1974a, b) have employed a pumice slurry and rubber cup followed by rinses in distilled water as a cleaning method prior to analyses for 69 elements, and Derise (1973), in a study involving Al, $\mathrm{Ca}, \mathrm{Cl}, \mathrm{Co}, \mathrm{Cu}, \mathrm{F}, \mathrm{Fe}, \mathrm{I}, \mathrm{K}, \mathrm{Mg}, \mathrm{Mn}$, $\mathrm{Na}, \mathrm{P}, \mathrm{Pb}, \mathrm{Se}, \mathrm{Sr}$, and $\mathrm{Zn}$ determinations, reports cleansing specimens in deionized water and polishing them with a stiff nylon brush (polishing medium, if any, unspecified). Battistone et al (1967) scrubbed specimens in deionized 
water with a nylon brush prior to Mn analysis.

One can only speculate regarding the potential for contamination of dental samples by procedures such as those cited above. The use of unprotected steel scaling instruments for the removal of surface debris presents a problem similar to that encountered in specimen extraction. Polishing the specimen with pumice, a substance that may be of volcanic or industrial origin, is in reality a grinding operation. Natural pumice contains $\mathrm{Si}, \mathrm{Al}, \mathrm{Ca}, \mathrm{Na}, \mathrm{K}, \mathrm{Fe}, \mathrm{Mg}, \mathrm{Ti}, \mathrm{Mn}$, and $\mathrm{P}$, listed approximately in decreasing order of occurrence, as major constituents [Karig and Glassley (1970)], and a variety of trace elements, including elements of the lanthanide series [Fin'ko (1964)]. That pumice may scratch the surface of enamel has been shown by Barnes et al (1971). Contamination from this or other polishing media, although probably confined to the surface zones of the specimen, remains problematical.

In any event, use of a nylon brush in conjunction with deionized water or with detergents of known composition is the safest means of cleaning specimens. Scaling instruments, if used, should be plastic or protected by plastic. (iii) Removal of the samples to be analyzed from the specimen.

The inorganic constituents of enamel and dentine are frequently subjects of investigation, as is the elemental content of different zones or layers of enamel, dentine, and root tissue. Removal of the tissue of interest from the bulk specimen involves procedures with a potential for contaminating the samples. This potential has been evaluated for some procedures and found to be important.

Steel, diamond, and carborundum cutting discs have been used to section teeth and separate t1ssue for analysis. Diamond wheels have been used to prepare sections of teeth for charged particle activation analysis of $\mathrm{Pb}$ by Malik et al (1974) and of C, Ca, and P by Stubbins et al (1967), and for electron microprobe analysis of $\mathrm{Ca}, \mathrm{Cl}, \mathrm{Mg}$, and $\mathrm{Na}$ by Besic et al (1969) and of $\mathrm{Pb}$ by Carroll et al (1972). Calonius et al (1965) separated enamel and dentine with a diamond disc 
prior to semiquantitative multielement X-ray fluorescence analysis, and Shapiro and coworkers (1973) have used diamond discs to prepare sections of teeth for removal of circumpulpal dentine for $\mathrm{Pb}$ analyses. A similar procedure was adopted by Steinnes et al (1974) in preparing enamel and dentine for neutron activation analysis for $\mathrm{La}, \mathrm{Sm}, \mathrm{Tb}$, and $\mathrm{Yb}$. Dolinsek et al (1975) also used a diamond disc to separate enamel from specimens prior to analysis for Al. A high-speed steel slotting saw was used by Losee et al (1974 a, b) to section teeth preparatory to removal of dentine for analyses of enamel samples involving 69 elements, and Hamilton (1971) used the same type of saw to prepare thin sections of teeth for neutron irradiation and analysis for U. Nixon and coworkers (1962, 1965, 1966, $1967 \mathrm{a}, \mathrm{b}, \mathrm{c})$ have used carborundum discs to section teeth and remove layers of enamel prior to analyses involving: $\mathrm{Cu}, \mathrm{Hg}, \mathrm{Mn}, \mathrm{Sb}$, and $\mathrm{Zn}$.

Losee and coworkers (1974a), although conceding the possibility that enamel samples may have been contaminated by elements present in the steel instruments used, conclude that their effect is probably unimportant in view of the proportion of enamel affected relative to the whole sample. Ahlberg et al (1976), however, rejected use of a (steel?) saw to prepare teeth for proton-induced X-ray emission analysis when, in a trial, they detected severe contamination of the sample (surface) by $\mathrm{Cu}, \mathrm{Fe}, \mathrm{Mn}$, and $\mathrm{Ti}$. Carborundum cutting discs were found by Nixon et al (1967c) to contribute no contamination for the elements studied, but Retief et al (1970a, 1971a,b), having ascertained the presence of $\mathrm{Al}, \mathrm{Cu}$, and $\mathrm{Mn}$ in a carborundum disc by neutron activation analysis, rejected its use as a means of separating enamel and dentine.

Diamond stones as well as a variety of dental burs have also been used for selectively removing tissue of interest from dental specimens. Brudevold et al' (1956a-c, 1963), Yoon et al (1960), Little et al (1966), and Steadman et a1 (1959), 
have used diamond stones to remove layers of enamel for analysis, and Nixon et al (1970) and Losee et al (1974a, b) have used tungsten carbide burs to remove dentine from enamel prior to analysis. Shapiro et al (1972) removed outer layers of enamel for analysis using dental burs; dental burs have also been used extensively for removing restorations and carious tissue prior to selection of tissue for analysis [e.g., Little et al (1966) and Stewart (1974)].

Evidence pertaining to contaminants introduced into samples through use of diamond stones and tungsten carbide burs has been documented for only one element. Battistone et al (1967) showed that the Mn content of enamel removed from teeth with tungsten carbide burs was roughly twice the content of enamel removed with polyethylene-protected instruments. Use of diamond stones also resulted in elevated Mn levels. Losee et al (1974b), however, have stated thit trial samples and analyses showed no contamination resulting from the use of slow-running tungsten carbide burs for the removal of dentine from enamel. samples. (broad spectrum, multi-element analyses).

Among the safest, but by no means generally applicable procedures, is the use of polythene-protected instruments for chipping enamel from the specimen, thereby separating enamel and dentine [Lundberg et al (1965a, b); Söremark and coworkers (1962a, b, 1964a, b); and Battistone et al (1967)]. Agate (mortar and pestle) has also been employed to separate enamel and dentine by chipping [Derise et al (1974a, b); Retief et al (1971a, b); and Dolinsek et a1 (1975)]; this procedure probably has little potential for contamination except for Si. As a final method of removing samples of interest from the specimen, we consider the flotation separation of enamel and dentine devised by Manly and Hiodge (1939). This is a widely used procedure whose potential for contamination is dependent on the purity of the chemicals (bromoform and acetone) used to effect separation. Inasmuch as organic solvents are easily purified by repeated 
distillation and since flotation separation does not employ large quantities. of these solvents, contamination of enamel and dentine samples by trace elements present in the solvents is probably negligible: [doubly distilled A. R. grade chloroform, for example, contains $\mathrm{Co}, \mathrm{Cu}, \mathrm{Fe}, \mathrm{Sc}, \mathrm{Sb}$, and $\mathrm{Zn}$ at $\mathrm{ppb}$ and sub-ppb levels (Robertson (1972))]. Contamination by bromine from ab or adsorbed bromoform is probable, however, a fact implied by the unusually high $\mathrm{Br}$ levels found in dental tissue subjected to flotation separation [Calonius et al (1965) and Hardwick et al (1967)].

(iv) Desiccation of the sample.

Trace element concentrations are frequently expressed on a dry. weight basis. Since the dry weight of dental hard tissues has been found to depend on drying time, temperature, and particle size (e.g., on whether the sample is reduced to powder before or after drying, etc.) by Burnett and Zenewitz (1958), apparent values of trace element concentrations will vary with the conditions" of the study. This. effect will be relatively more important for analyses of dentine, and by implication, whole tooth, because of their higher moisture content [Rowles (1967)]. At elevated temperatures $\left(>200^{\circ} \mathrm{C}\right.$, say) there is an additional risk of losses from the sample of the more volatile elements (negative contamination [Robertson (1972)]). The condition of the interior of the drying oven may constitute a potential source of sample contamination by a variety of elements and represents a variable not easily evaluated.

A wide range of temperatures has been employed to desiccate dental specimens, though the majority fall in the range $100-120^{\circ} \mathrm{C}$. Freeze-drying has also been used [Hamilton (1971)].

\section{(v) Grinding operations}

of all the physical manipulation involved in preparing a sample for analysis, none gives greater cause for concern with respect to sample contamination than 
grinding. During grinding, the sample is in intimate contact with the walls of the grinding vessel for prolonged periods at locally high pressures and, depending on conditions, temperature.

Some of the methods considered above for removing samples from dental specimens might properly be considered grinding operations; however, in the sense employed here, grinding is a procedure for reducing a sample to a (usually) fine powder. Powdered sample, whether required for analysis or not, is a prerequisite for flotation separation of enamel and dentine, and has been produced most often in this context. Asgar (1956), for example, crushed teeth in a mortar (nature of mortar not specified), screened the sample, and returned material not passing 100 mesh for further grinding until the whole sample passed 100 mesh. Hadjimarkos et al (1959, 1962) pulverized teeth (process unnamed) prior to flotation separation of dentine and enamel, and Se determination. Jackson et al (1959) pounded teeth to a fine powder in a stainless steel cylinder, while Needleman et al (1972) crushed teeth at liquid nitrogen temperatures in a Freezermf11 (Spex Industries, Inc.). Hardwick et al (1967) ground teeth in a steel ball mill, and Pearlman et al (1970) have employed a stainless steel hammer mill. Shaw et al (1959) pulverized teeth in a (steel) diamond mortar to pass 100 mesh, while Lockeretz (1975) employed an alumina mortar. Grinding enamel to pass 300 mesh, and enamel and dentine with agate mortars and pestles have been carried out by Losee et al $(1974 b, c)$, and by Derise et al (1974a) and Retief and coworkers (1970a, b, 1971a, b, 1974), respectively. Agate grinding media have also been used by Langmyhr et al $(1974,1975)$ to reduce whole teeth, first to 100 mesh, then to 270 mesh, and by Dolinsek et al (1975) to grind enamel samiples. Contamination from Mn by grinding enamel samples with a steel mortar and pestle has been documented by Nixon et.a1 (1966, 1967c). They report Mn levels five 
times the average of sound enamel for samples prepared in this manner. Losee et al (1974b) report that Si contamination from their agate mortar invalidated the data obtained for that element in a study involving the determination of 69 elements in ename1. In addition, Hardwick et al (1967) 1ist several elements that might be present as contaminants through use of, among other things, the steel ball mill. Cross contamination, i.e., contamination of a sample by residual amounts of previously ground samples (whether dental or not); has not to our knowledge been considered by investigators in this field (though in removing samples of enamel from teeth, Nixon et al $(1965,1966)$ report using a fresh carborundum cutting disc for each tooth).

In view of the limited data available in the dental literature on sample contamination by grinding, the results of a comprehensive study of sample contamination from grinding and sieving by Thompson and Bankston (1970) are summarized here. Of a variety of grinding media considered, these authors found that a boron carbide mortar (except for B), an agate mortar (Si was not measured), and Lucite grinding vials introduced little or no contamination when used to grind silica and calcium carbonate. Relevant to the use of tungsten carbide burs, whether for grinding or removing samples from specimens, was the finding that tungsten carbide grinding vials introduced significant amounts of Co and Ti into their samples. (Recall that tungsten carbide, a product of powder metallurgy, requires a binding agent for which co is commonly used).

While use of disposable Lucite grinding vials eliminates completely crosscontamination, we have found them ineffective in grinding whole teeth. of the mortars, agate is the least expensive and, from that standpoint, preferable to boron carbide. In grinding whole teeth, however, we have found preliminary crushing in an enclosed container (such as a diamond mortar faced with boron carbide), a desirable prerequisite to use of a mortar. 


\section{Chemical manipulation}

Preparation of derital samples for analysis may involve chemical treatment, including wet and dry ashing, sample dissolution, and chemical separation.

Wet and dry ashing constitute preconcentration techniques designed to destroy organic matter present in the sample, thus lowering the detection limits for inorganic constituents for an equivalent weight of sample. Ashing also serves to limit interferences in the ensuing analyses that may arise from organic matter in the sample, viz. in spark source mass spectrometry. Dry ashing of dental hard tissues is normally accomplished at elevated temperatures In a muffle furnace, but may also be carried out with a low temperature oxygen plasma. High temperature ashing was employed by Cruickshank (1949) on dentine and ename $1 \cdot\left(270^{\circ}\right.$ and $500^{\circ} \mathrm{C}$, respectively) prior to $\mathrm{Zn}$ analyses, and by Strehlow et al (1969) on whole tooth $\left(600-750^{\circ} \mathrm{C}\right)$ prior to $\mathrm{Pb}$ analysis. Brudevold et al (1956c), Steadman et al (1959), and Yoon et al (1960) ashed dental samples at $575^{\circ} \mathrm{C}$ prior to $\mathrm{F}$ analysis, while Hardwick et al (1967) ashed enamel and dentine at $600^{\circ} \mathrm{C}$ prior to multielement analysis. Results reported in the Soviet literature are based predominantly on (presumably high temperature) ashed samples [e.g., Ginzburg et al (1947); Nezhivenko (1961a, b); Nezhivenko et al (1967); Mamedov et al (1974); and Vikhm (1962)].

High temperature ashing is accompanied by several problems. Rowles (1967), for example, reports that thin layers of dentine are not completely ashed even after 40 hours at $600^{\circ} \mathrm{C}$, and has recommended ashing at temperatures approaching $1000^{\circ} \mathrm{C}$. Chemical changes in the inorganic fraction at high temperature introduce further uncertainty in the ash weight. In this respect, the problem is not unlike that accompanying desiccation of dental samples. Of more immediate concern, however, is the potential loss from the sample of the more volatile elements 
[Robertson (1972)]. Petrow and Cover (1965), for example, report loss of : Pb in bone samples ashed at temperatures exceeding $600^{\circ} \mathrm{C}$. Strehlow et al (1969), however, find $100 \%$ retention of $\mathrm{Pb}$ in whole teeth ashed overnight at $600^{\circ} \mathrm{C}$ followed by $1-2$ hours at a temperature not exceeding $750^{\circ} \mathrm{C}$. Corrosion products from the lining of the muffle furnace are a potential source of sample contamination which must also be considered [Robertson (1972)].

Low temperature plasma ashing, although less likely to involve loss of volatile elements, probably suffers from a dependence of ash weight on particle size. Aithough little data is available, the plasma is probably unable to penetrate dental hard tissue to any extent and hence, even at 325 mesh, much of the organic matrix remains unaffected. Zahradnik and Moreno (1975), however, have found that from between 60 and $70 \%$ of the organic matter in powdered enamel appears altered after plasma ashing. This technique does not appear to have beenused to ash samples prior to trace element characterization.

Wet ashing involves dissolution of the sample in a strongly oxidizing medium, e.g., nitric acid, and as such is one method of sample dissolution. Where dissolution alone is a goal, a variety of media can be used. Enamel has been dissolved in HCl [Brudevold et al (1955, 1956a-c); Steadman et al (1958, 1959); and Little et al (1966)] prior to emission spectroscopic determination of a number of elements. Derise et al (1974a, b) ashed enamel and dentine in nitric acid prior to atomic absorption analysis for $\mathrm{Co}, \mathrm{Fe}, \mathrm{K}, \mathrm{Mn}, \mathrm{Pb}$, and $\mathrm{Zn}$, and Dolinsek et al (1975) dissolved enamel in nitric acid (6N) prior to Al analysis. Enamel and dentine have been digested with a mixture of nitric and sulfuric acids, and mercuric nitrate by Hadjimarkos et al $(1959,1962)$. Whole tooth ash has been dissolved in $\mathrm{HBr}$ [Strehlow et al (1969)] and whole tooth in 5:2 and 9:1 mixtures of nitric and perchloric acids [Stewart (1974) and Lockeretz (1975)] and in 
concentrated hydrochloric acid [Ikeda (1967)]. Enamel has been dissolved in concentrated perchloric acid (62\%) by Helsby (1974). Needleman et al (1972) dissolved teeth in $70 \%$ perchloric acid to precipitate and separate protein from the inorganic material, while Shapiro et al $(1972,1973)$ have used $70 \%$ perchloric acid to digest various types of hard dental tissue (e.g., enamel, dentine, secondary dentine, and circumpulpal dentine). Kaneko et al (1974) digested whole teeth with hot nitric acid, evaporated the resulting solution to dryness, dry ashed it $\left(400-450^{\circ} \mathrm{C}\right)$, and dissolved the ash in concentrated hydrochloric acid prior to atomic absorption analysis for $\mathrm{Cd}, \mathrm{Cu}, \mathrm{Pb}$, and $\mathrm{Zn}$. Wet ashing techniques designed to solubilize the organic matrix, leaving the mineral fraction intact [reviewed by Rowles (1967)], are rarely used in studies of the trace constituents of teeth [see, however, Crowell, Jr. et ai (1934); Steadman et al (195.9); Lovaas et al (1968)].

Wet ashing and sample dissolution techniques require great care in the choice of reagents and containers for the preparation and storage of solutions. Robertson (1972) has tabulated common trace contaminants of hydrochloric, hydrofluoric, sulfuric, and nitric acids, as well as those present in a number of polymeric materials that might be leached by solutions stored in them, and Adams (1972) has reviewed glass containers from the standpoint of their chemical reactivity towards solutions stored in them. Containers have also been known to adsorb ions present in dissolved samples, resulting in misleading analyses [Robertson (1972)]. Litman et al (1975), for example, have shown losses of inorganic $\mathrm{Hg}$ at sub-ppm levels in $1 \mathrm{~N}$ nitric acid approaching $60-70 \%$ in solutions stored for prolonged periods (1.0-30 days) in polyethylene and teflon containers. Where sample dissolution is conducted at elevated temperatures, volatile elements may be lost. Derise (1973) however, reports good recovery for Co, Fe, $\mathrm{K}, \mathrm{Mn}, \mathrm{Pb}$, and $\mathrm{Zn}$ for nitric acid ashed samples of enamel and dentine. 
Chemical separation involves techniques designed to reduce specific interference from one or more elements in the sample, or to concentrate one or more elements, thus lowering their effective detection limits. Concentration techniques in trace analysis have been reviewed by Minczewski (1967), and more recently by $\mathrm{Tölg}(1972)$. Chelation of specific elements by an appropriate agent and subsequent extraction of the complex with an organic solvent is among the more commonly employed separation techniques in the analysis of dental hard tissues for trace constituents. It is particularly common in the case of $\mathrm{Pb}$ [Kaneko et al (1974); Needleman et al (1972); Rytömaa et al (1974); Stewart (1974); and Strehlow et al (1969)], but has also been employed for analysis of Cd [Iwakura (1972) and Kaneko et al (1974)], for Cu [Kaneko et al (1974)], and for $\mathrm{Zn}$ [Cruickshank (1949)]. To our knowledge, use of several chelating agents as described by Husler and Cruft (1969) for simultaneous recovery of Ag, Al, Au, $\mathrm{Be}, \mathrm{Cd}, \mathrm{Co}, \mathrm{Cr}, \mathrm{Fe}, \mathrm{Ga}, \mathrm{Ge}, \mathrm{Mo}, \mathrm{Ni}, \mathrm{Pb}, \mathrm{Sn}, \mathrm{Ti}, \mathrm{V}$, and $\mathrm{Zn}$ from a calcium sulfate matrix has not been attempted for dental tissues: Volatilization is employed in F analysis [Brudevold et al (1956c); Jackson et al (1959); Shaw et al (1959); Steadman et al (1959); and Yoon et al (1960)], and a combination of volatilization and precipitation in Se analyses [Hadjimarkos et al (1959, 1962)]. In addition, a variety of more or less complex procedures has been evolved for the separation of one or more radioisotopes in neutron activation analysis of dental tissues [Samsah1 et al (1961a, b); Nixon and co-workers (1960, 1962, 1965, 1966, 1967a, b); Retief et al (1974); Ikeda et al (1967); Sairenji et al (1961); Götte et al (1955); and Steinnes et al (1974)].

Chemical separation, to the extent that it involves manipulation of dissolved samples, is subject to sources of contamination mentioned earlier. Where a large number of steps (reagent additions, for example) are involved, the likelihood that some contamination will be introduced is increased. Reagent purity 
is not as critical in chemical manipulation following irradiation in activation analyses.

\section{Airborne contamination}

Sample contamination by atmospheric.'fallout' (i.e:, dust) is often overlooked as a source of analytical error; as limits of detection are further reduced through scientific and technical advances, however, particulate matter present in laboratory air will become an increasing source of concern. In the chain of events constituting sample preparation, the greatest risk for contamination by fallout is evident when the sample is in solution or in finely powdered form and exposed to the laboratory atmosphere -- for example, during filtration. The smaller the sample, the greater will be the effect. To illustrate, note that a particle of iron $45 . \mu \mathrm{m}$ in diameter (from an overhead ventilating unit, say) impinging on a $10 \mathrm{mg}$ sample will increase the sample iron content by $38 \mathrm{ppm}$. Airborne contamination problems have not generally been given recognition in the dental literature, though in at least one instance [Dolinsek et al (1975)] samples have been prepared under circumstances approaching "clean-room" conditions. Robertson (1972) and Paulhamus (1972) have reviewed the problem from the point of view of trace analysis. in general, and Zief and Nesher (1974) recommend procedures for upgrading the laboratory environment to control the problem.

\section{$\underline{\underline{1.3}}$ Sampling error}

Biological samples are seldom homogeneous with respect to the distribution of trace components. This inhomogeneity requires careful consideration of sampling error. Sampling error may be incurred for several reasons although here consideration is given only to error which results when a portion of tissue is analyzed for a measure of whole tissue content. Sampling error in the context of the collection of samples will be dealt with briefly in Chapter 2 . 
Where an entire sample (specimen) is dissolved and an aliquot of the solution subjected to analysis, sampling error is vanishingly small, providing that dissolution is complete and that the solution is homogeneous (i.e., a true solution). Inaccuracy in the relative size of the aliquot removed is a measurement error, not a sampling error. Consider, however, the analysis of a solid such as a powder. The entire sample (whole tooth, say) in powdered form represents a population S. Assume that $S$ is in reality the union of two mutually exclusive sub-populations, $S_{1}$ (enamel, say) and $S_{2}$ (dentine). Assume further that each unit (particle) of $S_{1}$ contains a trace component $X$ at a concentration $\mu_{1}$ and that, similarly, $S_{2}$ contains $x$ at a level of $\mu_{2}$. Then, ignoring density factors, and given that there are $N_{1}$ particles in $S_{1}$ and $N_{2}$. particles in $S_{2}$, the population mean, $\mu$, of $X$ in $S$ is given by:

$$
\mu=\Pi \mu_{1}+(1-\Pi) \mu_{2}
$$

where

$$
\Pi=\mathrm{N}_{1} / \mathrm{N} \text { and } \mathrm{N}=\mathrm{N}_{1}+\mathrm{N}_{2}
$$

Now, if a random sample of size $\underline{n}$ is drawn from $S$, with $\underline{n}_{1}$ units from $S_{1}$ and $\underline{n}_{2}$ units from $S_{2}$, our estimate of $\mu, \bar{x}$, based on an analysis of this random sample (our aliquot of powder) is given by

$$
\overline{\mathrm{X}}=\underline{\mathrm{p}} \mu_{1}+(1-\underline{\mathrm{p}}) \mu_{2}
$$

where

$$
\underline{p}=\underline{n}_{1} / \underline{n} \text { and } \underline{n}=\underline{n}_{1}+\underline{n}_{2}
$$

The variance of $\bar{x}$ may now be derived from fairly standard statistical theory [Sukhatme and Sukhatme (1970)].

$$
\begin{aligned}
& \text { Defining this variance, } \frac{\sigma_{\overline{\mathrm{X}}}^{2}}{\text {, as }} \\
& \qquad \sigma_{\overline{\mathrm{X}}}^{2}=\mathrm{E}\left(\overline{\mathrm{X}}^{2}\right)-\mu^{2}
\end{aligned}
$$


where E ( ) stands for the expectation of the enclosed quantity, one finds that

$$
\sigma_{\overline{\mathrm{x}}}^{2}=\Pi(1-\Pi)\left(\mu_{1}-\mu_{2}\right)^{2} \frac{\mathrm{N}-\underline{\mathrm{n}}}{\underline{\mathrm{n}}(\mathrm{N}-1)}
$$

The factor $(\mathrm{N}-\underline{\mathrm{n}}) /(\mathrm{N}-1)$ is known as the finite population correction and, although' not likely to be important for finely powdered samples where $\mathrm{N} \gg \underline{\mathrm{n}}$, is important for material in chunk form where $\mathrm{N}$ is small or where $\mathrm{N}=\underline{\mathrm{n}}$.

As an example, consider the $F$ analysis of a $10 \mathrm{mg}$ sample of whole tooth ground uniformly to $100 \mathrm{mesh}$. The approximate sample size is $0.7 \times 10^{3}$ particles, asșuming a uniform density of 2.7 for enamel and dentine (U.S. Sieve Series, ASTM E-11-70). Letting the F content of enamel and dentine be $204 \mathrm{ppm}$ and 627 ppm, respectively [Shaw et al (1959): Numbers are mean values for specimens obtained from Pakistan], and assuming that ename1 comprises $15 \%$ of the whole tooth, the sampling variance for a $10 \mathrm{mg}$ sample of 100 mesh whole tooth is $33 \mathrm{ppm}^{2}$, or $\sigma_{\overline{\mathrm{X}}}=5.7 \mathrm{ppm}$.

For very accurate and precise analytical techniques, the sampling error may be an order of magnitude or more larger than the (instrumental) analytical error. For analyses of whole teeth, however, the sampling error appears to be of minor importance for most elements when treated as a two-component system, viz., enamel and dentine. This may not be true for the analysis of whole enamel that has been removed by chipping, or of layers or sections of enamel; or for analyses on circumpulpal dentine or other ill-defined or difficulty removed zones of dental tissue.

Harris and Kratochvil (1974) have published some useful graphs for estimating required sample sizes for given $\sigma_{\overline{\mathrm{X}}}$ that are based on a variation 
of equation (4). For clarity such treatments of sampling error are greatly simplified. One may, however, derive estimates of sampling error for multicomponent systems (the treatment here was confined to the two-component case) that incorporate inhomogeneities in the subpopulations or that treat other complexities of real systems. Flanagan (1976) has recently taken a step in this direction.

In the preceding sections we have attempted to summarize techniques that have been employed for the preparation and analysis of dental hard tissues. Our focus has been on sample preparation with emphasis placed on the potential for contamination inherent in the cited procedures. We do not. mean to imply that contamination resulted in all cases or that it vitiates the reported analyses where it did result. Since most investigations are restricted to one or a few elements, contamination by elements other than those being measured is of no concern. Contamination is a much more serious problem in broad spectrum multielement analyses, however.

One of the difficulties we encountered in preparing this review was the paucity of detail in descriptions of experimental procedures. We; therefore, encourage authors to include as much detail as possible in preparing work for publication. 


\section{Chapter 2}

\section{Factors Affecting the Inorganic Composition}

of Dental Hard Tissues

In this chapter we will attempt to review the data uncovered in our survey of literature on the inorganic composition of dental hard tissues. In sections 2.1 and 2.2 , the data are tabulated, the table entries explained, and the methods we have employed in our statistical analyses presented and discussed. Sections 2.3 through 2.8 comprise topical coverage of these data, organized in terms of several major factors that have been found to affect the composition of teeth. Here we enumerate the conclusions that have been drawn from these data and, where possible, supplement them with our own analysis. Finally, for those who are not interested in details, we summarize our major conclusions in section 2.9 . 


\section{$\underline{2.1}$ Table format}

A total of 84 elements has been specifically sought in analyses of dental hard tissues. Of these, 62 have been listed in one or more references as present in dental samples within limits imposed by analytical techniques employed, although only 43 have been detected/ quantified by two or more analytical procedures. Given the formidable scope of these data and the (largely unknown) interests of those who may have use of them, as much detail as space permits has been included in their tabulation. Table II includes data on 60 elements: The two for which no data are provided are hydrogen and oxygen. Also not included, are elements for which the only data available show concentrations below detection limits. The column entries in this table are described below.

Table II

Column 1, Serial number. Column 2, Tissue type. The principal tissue types appearing in this tabulation are D--dentine, E--enamel, P--pulp, R--root, and W--whole tooth. Entries may be further qualified by one or more words: E, Inner, for example. Information regarding tooth type and donor sex may be included when known. Abbreviations of the type B, Bl, C, I, M, Ml, and M3 refer to the position of specimens in the human dentition (B for bicuspids, Bl for first bicuspids, etc.). Note has been made when the data refer specifically to unerupted teeth and when the teeth are deciduous. Reference to donor sex is spelled out. A blank entry means 
that the tissue type has not been explicitly stated or cannot be inferred from the text of the article or abstract from which the data were obtained. Column 3, Provenance. Sample provenance, when explicitly known, is listed in this column. Knowledge of sample provenance, however, does not necessarily imply that samples have been obtained from life-long residents of the named place. In the absence of explicit data, knowledge of the country in which a study was conducted may provide (limited) information on provenance, since teeth obtained for study are most often of local origin. This information is entered in parentheses in this column. Column 4, Age. When known, the age (or an age range) of individuals providing teeth for study is entered in this column. Occasionally, a calendar year or years are entered, referring generalily to deceased populations from the given period.

Column 5, Samples. In most cases, this refers to the number of samples or analyses that the remaining statistics in a given row are based on. Unfortunately, this number is not always an accurate measure of the number of analyses on which the sample mean is based.

Column 6, mean. The sample mean is generally the arithmetic mean of data above detection limits $\overline{\mathrm{x}}=\sum \mathrm{x}_{\mathrm{j}} / \underline{n}$, where the summation is over all such data); however, where the author has arithmetically averaged logarithmically transformed data and then transformed the result back to a concentration, the tabulated, mean is a geometric mean and has been appropriateiy footnoted. If the underlying (statistical) distribution of the data is log-normal, this number is a sample. estimate of the median. 
Occasionally, data we have found in our survey are stratified:

They are classified with the aid of some external variable such as age or residence. In an effort to reduce the size of our tabulation, this stratification is sometimes removed and an overall mean tabulated. If there are $\underline{k}$ classes and $\underline{n}_{\underline{j}}$ samples in each class, the overall mean is

$$
\bar{x}=\sum_{j=1}^{k} \underline{n}_{j} \overline{x_{j}} / \sum_{\underline{j}=1}^{\frac{k}{n_{j}}}
$$

Column F, Error: The standard error is the sample estimate of the standard deviation of the sample mean,

$$
s_{\bar{x}}=\left\{\frac{1}{\underline{n}(\underline{n}-1)} \sum_{j=1}^{n}\left(x_{j}-\bar{x}\right)^{2}\right\}^{\frac{1}{2}}
$$

except where the entry in column 6 is a geometric mean; then the standard error is derived from a first-order Taylor series expansion of the standard error of the log transformed data. Hence, the entry in this column has the same physical units as the tabulated mean.

When an overall mean, obtained by removing stratification with respect to an external variable is tabulated in column 6 , the standard error has been computed as follows:. If there are $\underline{k}$ classes and $\underline{n}_{j}$ samples in each class with mean $\bar{x}_{\underline{j}}$ and standard error $s_{\bar{x}_{\underline{j}}}$ the standard error of the overall mean is given by 


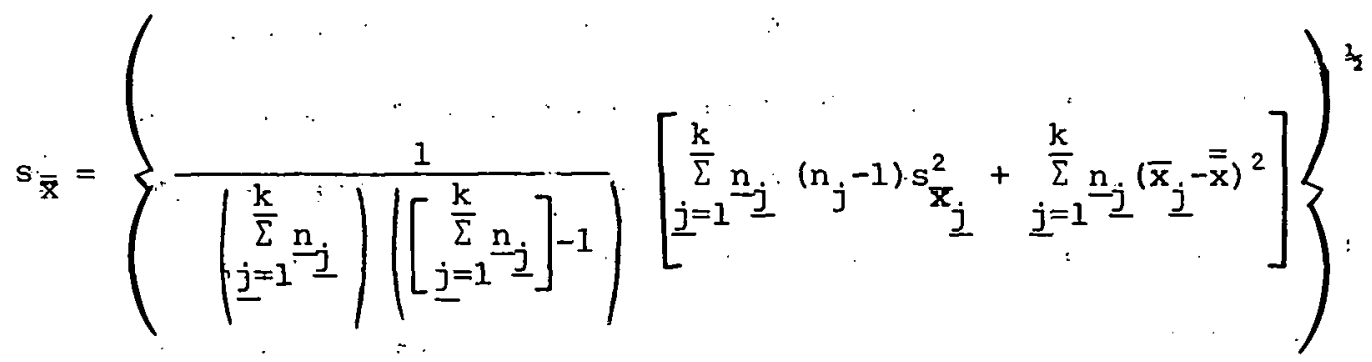

This is the same standard error that would have been obtained had the data not been stratified to begin with.

The standard error is tabulated when known and is based only on data above detection limits. We caution, however, that entries in this column may occasionally be standard deviations of the data about their mean owing to imprecision in the use of descriptive statistical terms in the literature.

Column 8 , Median. The median is that number above and below which $50 \%$ of the data lies and is a nonparametric statistic. It is tabulated when known and is generally based on all of the data, including those which are below instrumental detection limits.

Columns 9 and 10, Min and Max. When available; the minimum and maximum together are the range covered by the data. They are also used to provide a measure of the range (not necessarily inclusive) of data that are not in themselves the results of analyses of single samples and that are otherwise too detailed for inclusion in this tabulation (cf work by Brudevold and co-workers). The minimum and maximum are also employed for tabulating semi-quantitative data. 
Column 11, Exp. All compositional data are based on weights. The entry. in this column is the number of decimal places the decimal point of the mean, error, median, minimum and maximum must be shifted in a given row to bring the corresponding numbers to units of gram/gram. An exponent of -06 , for example, means the data tabulated are in units " of $\mu \mathrm{g} / \mathrm{g}$ or $\mathrm{ppm}$. No attempt has been made to tabulate the basis of these units, viz. ash weight, dry weight, etc. Though this information is sometimes available, in many cases it is not clear what basis an author has used to express his results.

Column 12, Reference. The data tabulated have been obtained from the cited work. The reference is footnoted to indicate whether the citation is based on an abstract, whether additional data, not tabulated, may be found in the cited study, and/or whether the results of individual analyses have been tabulated by the author of the cited study. A serious attempt has been made to reduce duplication in this tabulation that would result if complete entries were made for data that have been published on two or more occasions. References to duplicate work are present in the table, however, as single entries which contain no compositional data, but that are otherwise footnoted to the effect that additional data may be found in the cited work.

Special meaning has been assigned to certain combinations of values in our tabulation of compositional data: (i) If entries exist under the headings of samples and max, but not under any of the remaining headings (mean, error, median, and $\mathrm{min}$ ), then the concentration was below the instrumental detection limit (given by max) for the specified number 
of samples. (ii) Where no compositional data is provided, the cited reference contains only statements to the effect that the named element was detected, the compositional data were not available to us (viz. citations based on abstracts), the data were not in a form suitable -for inclusion in our tabulation, or, as described above, the cited reference is a duplication of work published in another article by the same author. .

The data in Table II are sorted alphabetically by atomic symbol and for a given symbol, by principal tissue type. In addition, entries for a given element and tissue from the same literature reference are grouped together. 


\section{TABLE II.}

\section{THE ELEMENTAL COMPOSITION OF TEETH}




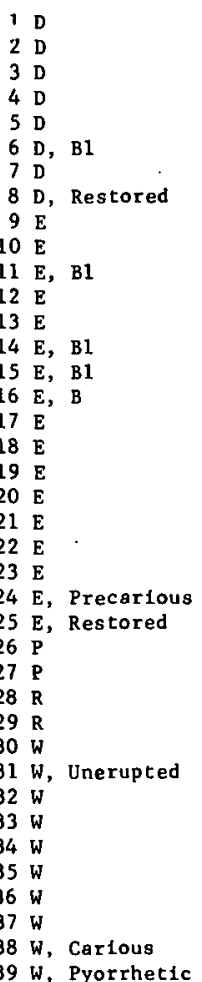

A1

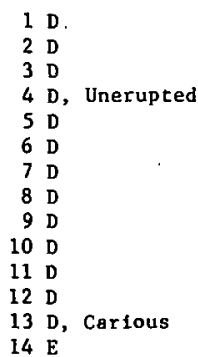

(Great Britain)

(Japan)

(South Africa)

(South Africa)
Stockholm

(U.S.A.)

(Great Britain)

New Zeal and

(South Africa)

(Sweden)

(U.S.A.)

(U.S.A.)

\section{Dacertown}

Indian Kno11, KY

Pueblo Bonito, N.M.

(U.S.A.)

(U.S.S.R.)

Watertown

(Sweden)

Japan)

Indian Knoll, $K$

Pueblo Bonito, N.M.

(Finland)

(Norway)

(Norway)

(U.S.S.R.)

(U.S.S.R.)

(U.S.S.R.)

(U.S.S.R.)

(U.S.S.R.)

(U.S.S.R.)

(Great Britain)

(Italy)
(Italy)

(Italy)
(South Africa)

(South Africa)

Virginia

Virginia
Virginia

Virginia

(U.S.A.)

(U.S.A.)
(Italy)

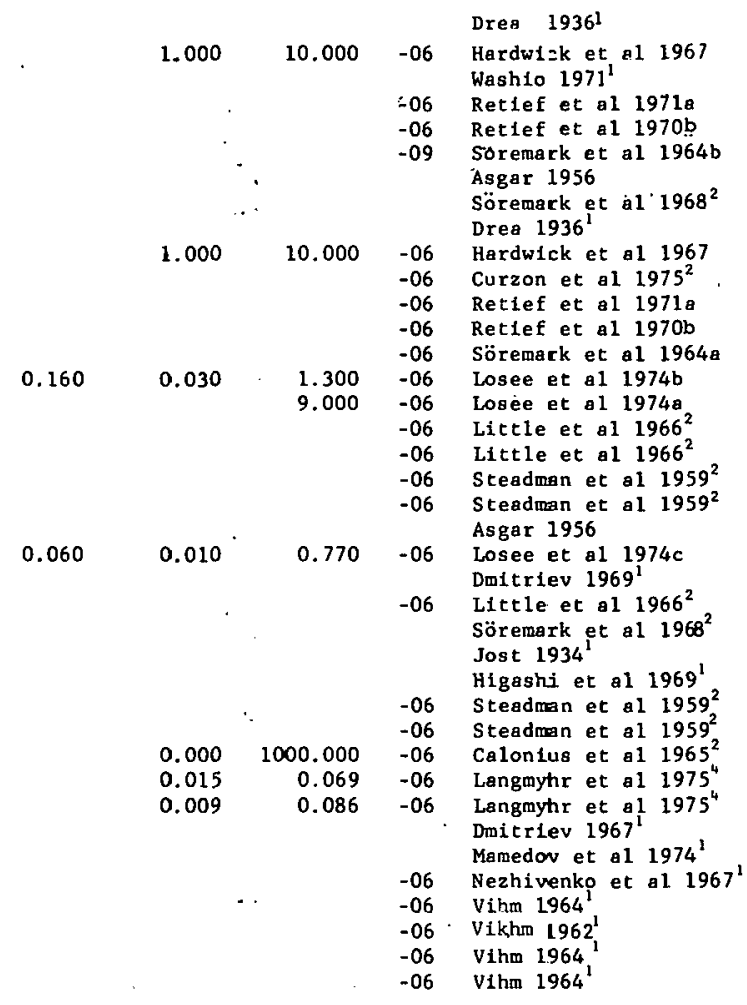

$10.000 \quad 100.000$

Drea $1936^{1}$

Hardwick et al 1967 risippaudo 1963 Grippaudo 1963 Retief et al 1970 Derise et al 1974b Derise et al 1974b Derise et $a \frac{1}{2} 1974 b$ Derise 1973 Aegar 1956 Grippaudo 1963
Drea $1936^{1}$ 


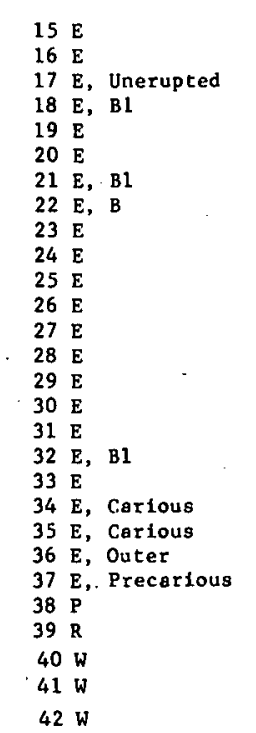

$\begin{array}{ll}\text { (Great Britain) } & \\ \text { (Italy) } & \\ \text { (Italy) } & \\ \text { New Zealend } & 11 \\ \text { (South Afr1ca) } & \\ \text { (South Africa) } & \\ \text { (U.S.A.) } & <20 \\ \text { (U.S.A.) } & <20 \\ \text { Virginia } & 10-12 \\ \text { V1rginia } & 13-16 \\ \text { Virg1nia. } & 17-24 \\ \text { VIrg1n1a } & >24 \\ \text { (U.S.A.) } & \\ \text { Rochester } & \\ \text { Watertown } & \\ \text { (U.S.A.) } & \\ \text { Rochester } & \\ \text { (U.S.A.) } & \\ \text { (Yugosiavia) } & <20 \\ \text { (Italy) } & \\ \text { Rochester } & \\ \text { (Yugoslavia) } & \\ \text { Watertown } & \\ \text { (Germany) } & \\ \text { Indian Kno11, KY. } & 2900 \text { BC } \\ \text { (Italy) } & \\ \text { (U.S.S.R.) } & \\ \text { (U.S.S.R.) } & \end{array}$

\begin{tabular}{|c|c|c|c|c|c|c|c|}
\hline & & \multirow{2}{*}{$\begin{array}{l}10.00 c \\
\ddots\end{array}$} & 100.000 & -06 & \multirow{2}{*}{$\begin{array}{l}\text { Hardulck et a1 } 1967 \\
\text { Grippaudo } 1963 \\
\text { Grippaudo } 1963\end{array}$} \\
\hline & & & & & & & \\
\hline 36 & 17.600 & 3.900 & & & & -06 & Curzon et al $1975^{2}$ \\
\hline 6 & 236.300 & 9.400 & & & & -06 & Retief et al $1970 b$ \\
\hline 8 & 86.130 & 4.540 & & & & -06 & Retief et al 1971a \\
\hline 28 & 12.500 & 2.940 & 5.600 & $1.50 \mathrm{G}$ & 70.000 & -06 & Losee et a1 1974b \\
\hline 93 & 74.700 & 10.040 & 47.000 & & 325.000 & -06 & Losee et a1 1974a \\
\hline 39 & 86.600 & 2.500 & & & & -06 & Derise et al 1974b \\
\hline 47 & 93.300 & 2.300 & & & & -06 & Der1se et a1 1974b \\
\hline 43 & 88.300 & 2.400 & & & & -06 & Derise et al 1974b \\
\hline 44 & 94.400 & 2.400 & & & & -06 & $\begin{array}{l}\text { Derise et al 1974be } \\
\text { Derise } 1973^{2}\end{array}$ \\
\hline & 16.000 & & & & & -06 & Litrle et al $1966^{2}$ \\
\hline 137 & 23.100 & & & & & -06 & $\begin{array}{l}\text { L1tcle et al } 1966^{2} \\
\text { Asgar. } 1956\end{array}$ \\
\hline & 32.000 & & & & & -06 & Johansen 1965 \\
\hline 56 & 14.000 & 1.510 & 10.000 & $1.50 \mathrm{C}$ & 54.000 & -06 & Losee et al 1974c \\
\hline 8 & 6.240 & 1.003 & & 3.900 & 11.500 & -06 & Dolinsek et al $1975^{4}$ \\
\hline & 93.000 & & & & & -06 & Johansen 1965 \\
\hline & & & & 12.000 & 39.000 & -06 & Dolinsek et a $1975^{4}$ \\
\hline 137 & 43.400 & & & & & -06 & Litcle et al $1966^{2}$ \\
\hline & 80.000 & & & & & -06 & Steadman et al $1959^{2}$ \\
\hline & & & & & & & $\begin{array}{l}\text { Peruccind } 1949^{\prime} \\
\text { Mamedov et a1 } 1974^{1} \\
\text { Zakson } 1968^{1}\end{array}$ \\
\hline
\end{tabular}

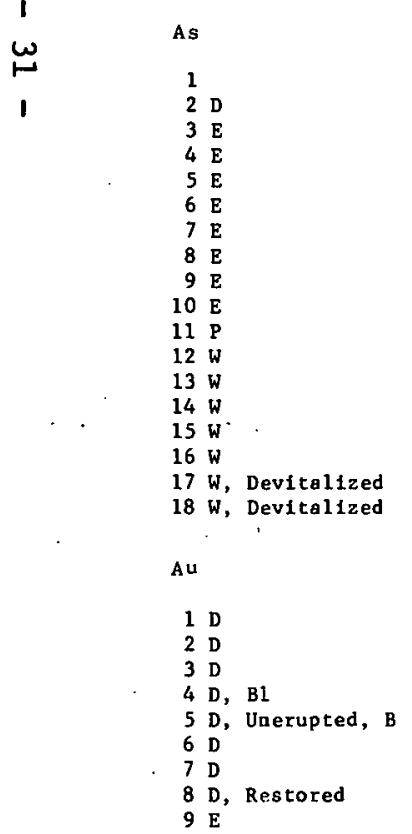
(Japan)
(Sreat Britain)
Denmark
Denmark
Denmark
Denmark
Denmark
(Great Britain)
(Great Britain)
(Japan)
(Great Britaln)
(Japan)
(U.S.S.R.)
(Germany)

(Great Britain)

(South Africa)

(South Afr 1ça)

(Sweden)

(Sweden)

(Sweden)

(Great Britain)

$\begin{array}{lrll}1100-1400 \mathrm{AD} & 14 & & \\ 1500-2300 \mathrm{BC} & 10 & & \\ 1850 \mathrm{AD} & 5 & & \\ 1970 \mathrm{AD} & 12 & & \\ 400 \mathrm{BC}-200 \mathrm{AD} & 8 & & \\ 800-1100 \mathrm{AD} & 3 & & \\ & 75 & 0.070 & 0.085 \\ & & & \\ & 4 & 0.022 & 0.006 \\ & 25 & 0.060 & \\ & 5 & 0.420 & 0.190 \\ & & 0.100 & \\ & 5 & 0.100 & \\ & 6 & 5.560 & 1.910 \\ & & & \\ & & & \\ & & & \\ 15-16 & 8 & 0.070 & 0.040 \\ 14-25 & 8 & 0.070 & 0.010 \\ & 15 & 0.030 & 0.010 \\ & & & \\ & & & \\ & & & \end{array}$

\begin{tabular}{|c|c|c|c|}
\hline & & & Otani $1957^{1}$ \\
\hline 10.000 & 100.000 & -06 & Hardwick et al 1967 \\
\hline 0.001 & 0.239 & .06 & Raswussen 1974 \\
\hline 0.010 & 0.406 & -06 & Rasmussen 1974 \\
\hline 0.029 & 0.105 & -06 & Rasmussen 1974 \\
\hline 0.001 & 0.008 & -06 & Rasmussen 1974 \\
\hline 0.001 & 0.308 & -06 & Rasmussen 1974 \\
\hline 0.007 & 0.020 & -06 & Rasmussen 1974 \\
\hline 0.003 & 0.630 & -06 & N1xon et al $1967 \mathrm{c}$ \\
\hline 10.000 & 100.000 & -06 & $\begin{array}{l}\text { Hardwick et al } 1967 \\
\text { H1gash1 et al } 1969^{1}\end{array}$ \\
\hline 0.012 & 0.036 & -06 & Gotte et. al $1955^{4}$ \\
\hline & 0.145 & -06 . & Nixon et al 1960 \\
\hline 0.080 & 1.150 & -06 & Sairenj1 et al $1961^{4}$, \\
\hline & & -06 & Nezh1venko et \\
\hline & & & V1khm $1963^{\prime}$ \\
\hline 0.012 & 13.730 & -066 & Gotte et al $1955^{4}$ \\
\hline & & & Sairenji et a1 $1961^{4}$ \\
\hline
\end{tabular}

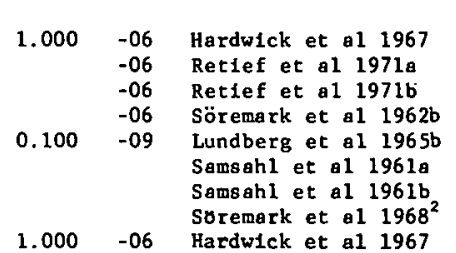


TISSUE TYPE

$10 \mathrm{E}$

$11 \mathrm{E}, \mathrm{B} 1$

$13 \mathrm{E}$, Unerupted, $\mathrm{B}$

$14 \mathrm{E}$
$15 \mathrm{E}$

$16 \mathrm{E}$, Restored

$18 \mathrm{~W}$

$19 \mathrm{~W}$, Restored

$2 \mathrm{E}$

$\begin{array}{lll}3 & \mathrm{E}, \mathrm{B} 1 \\ 4 & \mathrm{E}, & \mathrm{B} 1\end{array}$

$5 \mathrm{E}, \mathrm{B}$

$6 \mathrm{E}, \mathrm{B}$

$8 \mathrm{~W}$

$9 \mathrm{~W}$, Restored

Ba.

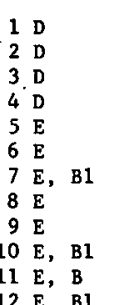

$11 \mathrm{E}, \mathrm{B}$
$12 \mathrm{E}, \mathrm{B} 1$

Be

1 E, B

$2 \mathrm{E}, \mathrm{Bl}$
$3 \mathrm{E}, \mathrm{BI}$

Bi

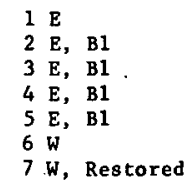

Br
PROVENANCE

(South Africa)

(South Afr

(Sweden)

(Sweden)

(Sweden)

(sweden)

(Japan)

(U.S.A.)

(Great Britain)

(Great Britain)

New Zealand
(U.S.A.)

(U.S.A.)

(U.S.A.)

(Italy)

(O.S.A.)

(Freat Britain)

(South Africa)
(South Africa)

(Greet Britaln) New Zealand

(South Africa)

(U.S.A.)

(U.S.A.)

$<20$
$<20$
$<20$

11
$<20$
$<20$
$<20$

$15-15$
$14-2 j$

AGE

MEAN

ERRO

MEDIAN

MDN

$\begin{array}{lll}7 & 0.110 & 0.070 \\ 7 & 0.110 & 0.030\end{array}$

$\begin{array}{lll}7 & 0.110 & 0.030 \\ 15 & 0.020 & 0.010\end{array}$

-06 Rerlef et al 1971a

-06 Retief et al 1971b

Lundberg et al 1965 a Samsahl et al 1961a Samsah1 et a $1961 \mathrm{~b}$ H1gashi et $811^{1969^{1}}$ Dmitriev $1967^{1}$ Fielda et al $1950^{1}$

.000

1.000

10.000

$\begin{array}{rrrr}36 & 11.800 & 2.260 & \\ 28 & 5.000 & 1.510 & 2.400 \\ 93 & 20.300 & 2.590 & 12.000 \\ 56 & 18.200 & 2.650 & 9.100\end{array}$

0.500

0.500

39.000

141.000

-06
-06
-06
-06
-06

dw1ck et a1 1967 Hardwick et al 1967 Cureon et al 1975 Losee et al 1974b Losee et al 1974c Pieruccini $1949^{\circ}$ Fields et al 1950

Drea $1936^{1}$

$10.000 \quad 100.000 \quad-06 \quad$ Hardwick et al 1967 Ret1ef et al 1971a Ret1ef et al 1970b

$\begin{array}{ll}129.050 & 54.690 \\ 129.050 & 20.720\end{array}$

$36 \quad \begin{array}{rr}13.900 & 2.250\end{array}$

$\begin{array}{lll}7 & 125.110 & 23.680\end{array}$

\begin{tabular}{l}
$4.200-8.970$ \\
\hline
\end{tabular}

$\begin{array}{rr}0.600 \\ 0.750 & 3.400\end{array}$ $\begin{array}{rr}0.750 & 13.000 \\ 0.510 & 4.700\end{array}$

10.000

100.000

$-06$ Hardwick et al 1967 Curzon et al 1975 Retief et al 1971a Ret1ef et al 1970b $\begin{array}{llll}0.800 & 13.000 & -06 & \text { Losee et al 1974b } \\ 0.800 & 44.000 & -06 & \text { Losee et a1 1974a } \\ & 17.000 & -06 & \text { Losee et a1 1974 C }\end{array}$ $\begin{array}{lllll}0.800 & 13.000 & -06 & \text { Losee et al 1974b } \\ 0.800 & 44.000 & -06 & \text { Losee et a1 1974a } \\ & 17.000 & -06 & \text { Losee et a1 1974 C }\end{array}$

(U.S.A.)

(U.S.A.)

$<20$
$<20$

$<20$

$93 \quad 0.310$

0.060

$10-0.090$

$\begin{array}{ll}2.500 \\ 0.010 \\ 0.990 & -066\end{array}$

0.010
0.970

Losee et al 1974a Losee et al 1974c Losee et al $1974 c$

(Japan)

(U.S.A.)

(U.S.A.)

(U.S.A.)

(Japan)

$<20$
$<20$
$<20$
$<20$

24
4
24

0.000

(Finland)
(Great Britain)

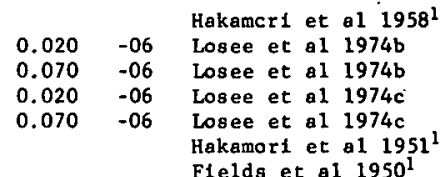

$\begin{array}{rrrr}1.000 & 50.000 & -02 & \text { Calonius et al } 1965^{2}\end{array}$ 


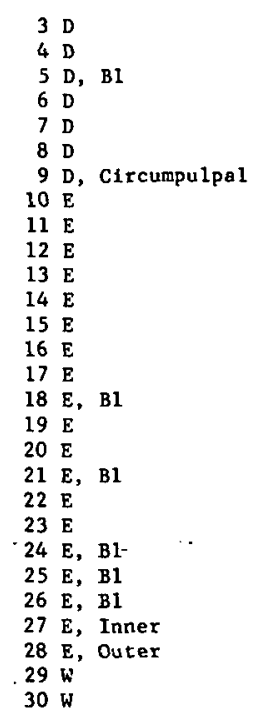

(South Africa)
(South Africa)
(Sweden)
(Sweden)
(Sweden)
(Sweden)
(Sweden)
Denmark
Denmark
Denmark
Denmark
Denmark
Denmark
(Finland)
(Great Britain)
New Zealand
(South Africa)
(South Africa)
(Sweden)
(Sweden)
(Sweden)
(U.S.A.)
(U.S.A.)
(U.S.A.)
(Sweden)
(Sweden)
(South Africa)
(South Africa)

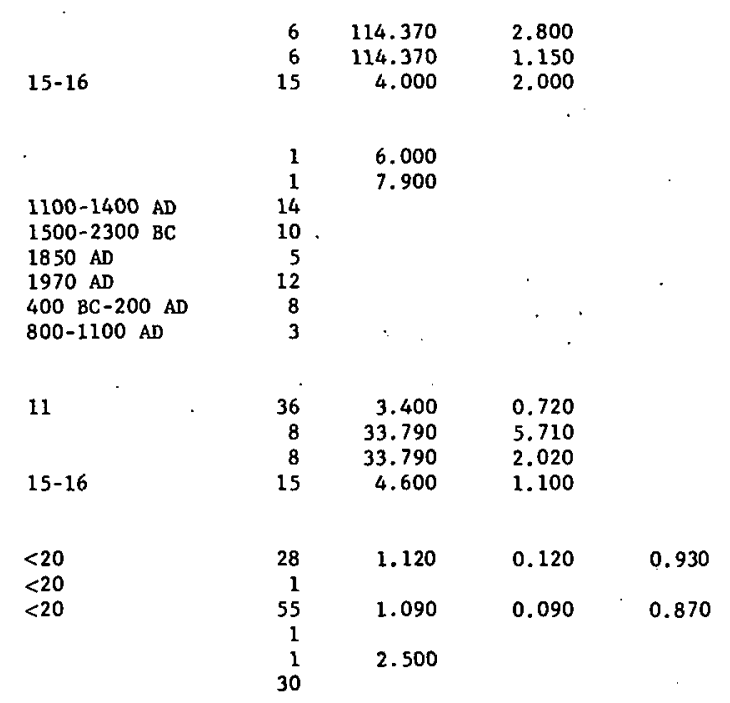

$\begin{array}{rrr} & & -06 \\ & & -06 \\ & & -06 \\ 3.500 & & \\ 3.703 & 7.300 & -06 \\ 3.800 & 4.600 & -06 \\ 1.50 J & 4.400 & -06 \\ 0.870 & 4.400 & -06 \\ 3.809 & 4.600 & -06 \\ 0.010 & 7.100 & -06 \\ 10.001 & 1.000 & -02 \\ & 100.000 & -06 \\ & & -06 \\ & & -06 \\ & & -06 \\ 0.320 & & \\ & 2.600 & -06 \\ & 0.100 & -06 \\ & 2.900 & -06 \\ & 2.200 & -06 \\ & & -06\end{array}$

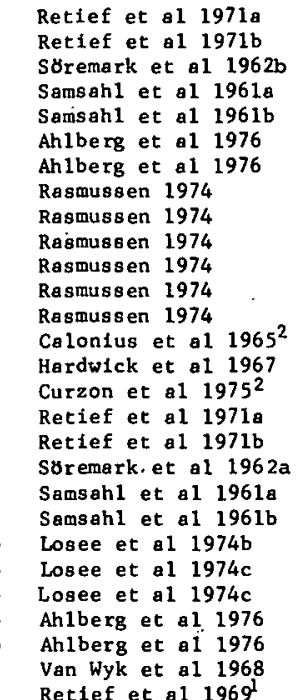

$2 \mathrm{D}$, Inner

$3 \mathrm{E}$, Outer

$5 \mathrm{~W}$, Deciduous

(Great Britain)

(Great Britatn)

(Gerneny)

(Germeny)

ca

(U.S.A.)

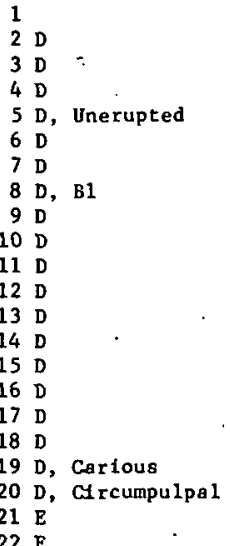

(Finland)

(Italy)

(South Africa)

(South Africa)

(Sweden)

(Sweden)

(Sweden)

virginla

Virginia

virginia

Virginie

(U.S.A.)

U.S.

(Italy)

$10 \quad 1.580 \quad 0.050$

Drea $1936^{1}$

tubbins et al 19674 Stubbins et al 1967 Gassman

Sasman

Besic et a1 $1969^{4}$

Calonius et al $1965^{2}$ Grippaudo 1963

Gr1ppaudo 1963

Retief et al 1970a

Soremark et al 1962b

Somark et al $1962 \mathrm{~b}$

Samsahl et al 1961b

Ah1berg et al 1976

Derise et al 1974a

Derise et al 1974a

Derise et al 1974a

Derise et $\& 1$ 1974a

Derise $1973^{2}$

Asgar 1956

Asgar 1956

Ahlberg et al 1976
Drea $1936^{1}$

(Finland)

-02 Calonius et a1 $1965^{2}$ 
TISSUE TYPE

PROVENANCE

(Italy)

(South Africa)

South Africa

(Sweden)

(Sweden)

Virginia

Virginia

Irginia

(U.S.A.)

Greec

U.S.

Rochester

(Italy)

(Sweden)

(Sweden)

(Finland)

(Germany)

(Germany)

(Germany)

Hachijo Is land

me

(South Africa)

(South Africa)

(U.S.A.)

(U.S.A.)

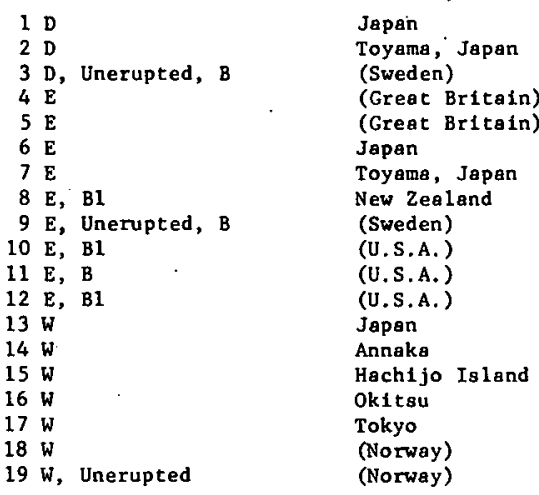

$14-25$

11 14-25

$14-25$
$<20$

$<26$

orway)

$\begin{array}{ll}1 \mathrm{E}, & \mathrm{B} 1 \\ 2 \mathrm{E}, \mathrm{BI} & \end{array}$

(U.S.A.)
(U.S.A.)
MI

MAX EXP

$\begin{array}{rrrr}7 & 31.690 & 0.330 & \\ 8 & 37.030 & 0.560 & -02 \\ 15 & 37.400 & 1.000 & -02 \\ & & & \\ 39 & 36.200 & 0.500 & -02 \\ 47 & 37.300 & 0.400 & -02 \\ 45 & 37.400 & 0.500 & \end{array}$
Grippaudo 1963

Retief et al 1970

sörenark et al 1962

Samsahl et al 1961a

Samahl et al 1961a

Derise et al 1974a
Derise et al 1974a

Derlse et al 1974a

Derise et al 1974a

Derise $1973^{2}$

Asgar 1956

Asgar 1956

Grippeudo 1963

Grippaudo 1963

Ahlberg et al 1976

Ahlberg et $21 \quad 1976$

Calonius et al $1965^{2}$

Gassmann ${ }^{1}$

Gessmenn 1

Gassmann ${ }^{1}$

Kaneko $1972^{4}$
Kaneko $1972^{4}$

Kaneko $1972^{4}$
Kaneko $1972^{4}$

Keneko 1972

Van Wyk et al 1968

02 Crowell, Jr. et a1 $1934^{4}$ Fields et al $1950^{1}$ Fields et al $1950^{1}$

$139 \quad 37.500 \quad 9.800$

$27.500 \quad 12.900$

30.800
29.000

$\begin{array}{rcc}15 & 0.099 & 0.092 \\ 18 & 0.404 & 0.230 \\ 10 & & \\ 6 & & \\ 18 & 0.135 & 0.166 \\ 73 & 0.224 & 0.096 \\ 36 & 3.300 & 0.590 \\ 10 & & \\ 28 & 0.510 & 0.120 \\ 93 & 14.900 & 3.050 \\ 56 & 0.990 & 0.150 \\ 32 & 0.120 & 0.060 \\ 17 & 0.550 & 0.310 \\ 12 & 0.080 & 0.030 \\ 19 & 0.100 & 0.080 \\ 8 & 0.405 & 0.123 \\ 6 & 1.020 & 0.324 \\ & & \end{array}$

0.092

.166

0.096
0.590

0.120
3.050
0.150

0.220

7.400
0.630

0.120

0.500
0.070

0.070
0.080

0.324

1.000


0.030
0.030
0.010
0.020
0.030
0.030
0.090
0.070

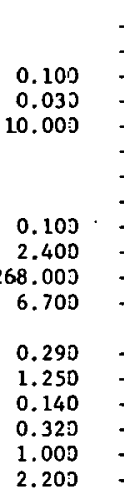

Iwakura $1972^{2}$ Iwakura $1972^{2}$ Lundberg et al 1965b Nixon et al 1967c Hardwick et al 1967 Iwakura $1972^{2}$ Twakura $1972^{2}$ Lundberg et a1 1965 a Losee et al $1974 \mathrm{~b}$ Losee et al 1974 e

Losee et al $1974 \mathrm{c}$

Kaneko et al 1974

Kaneko $1972^{4}$

Kaneko $1972^{4}$

Kaneko 19724

Kaneko $1972^{4}$
Langmyhr et al $1974^{4}$

$\begin{array}{lll}1.000 & -06 & \text { Langmyhr et a1 } 1974^{4} \\ 2.200 & -06 & \text { Langmyhr et al } 1974^{4}\end{array}$

$\begin{array}{ll}0.070 & 0.020 \\ 0.070 & 0.020\end{array}$

$0.190 \quad-06 \quad$ Losee et a1 $1974 \mathrm{~b}$ 


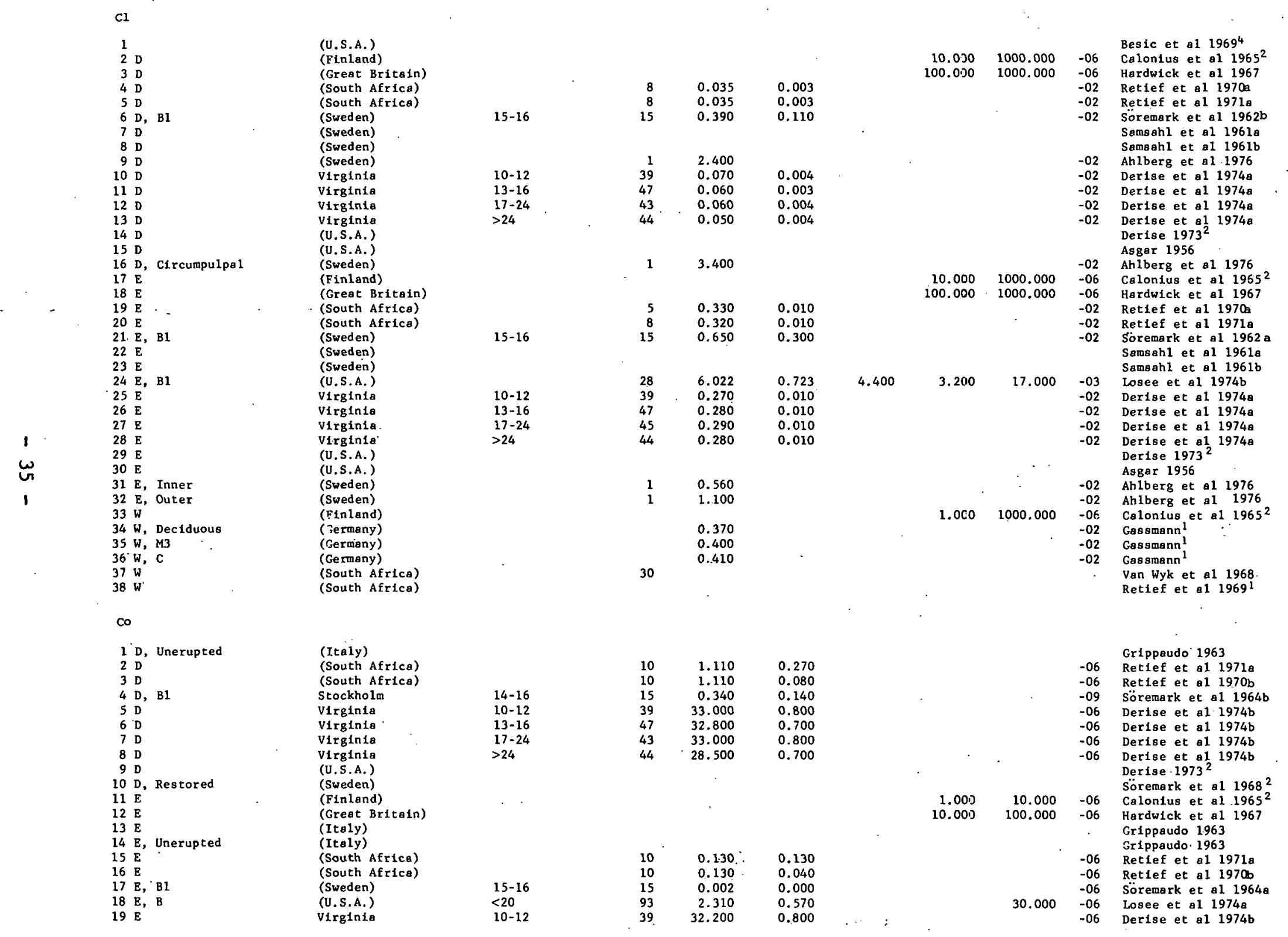




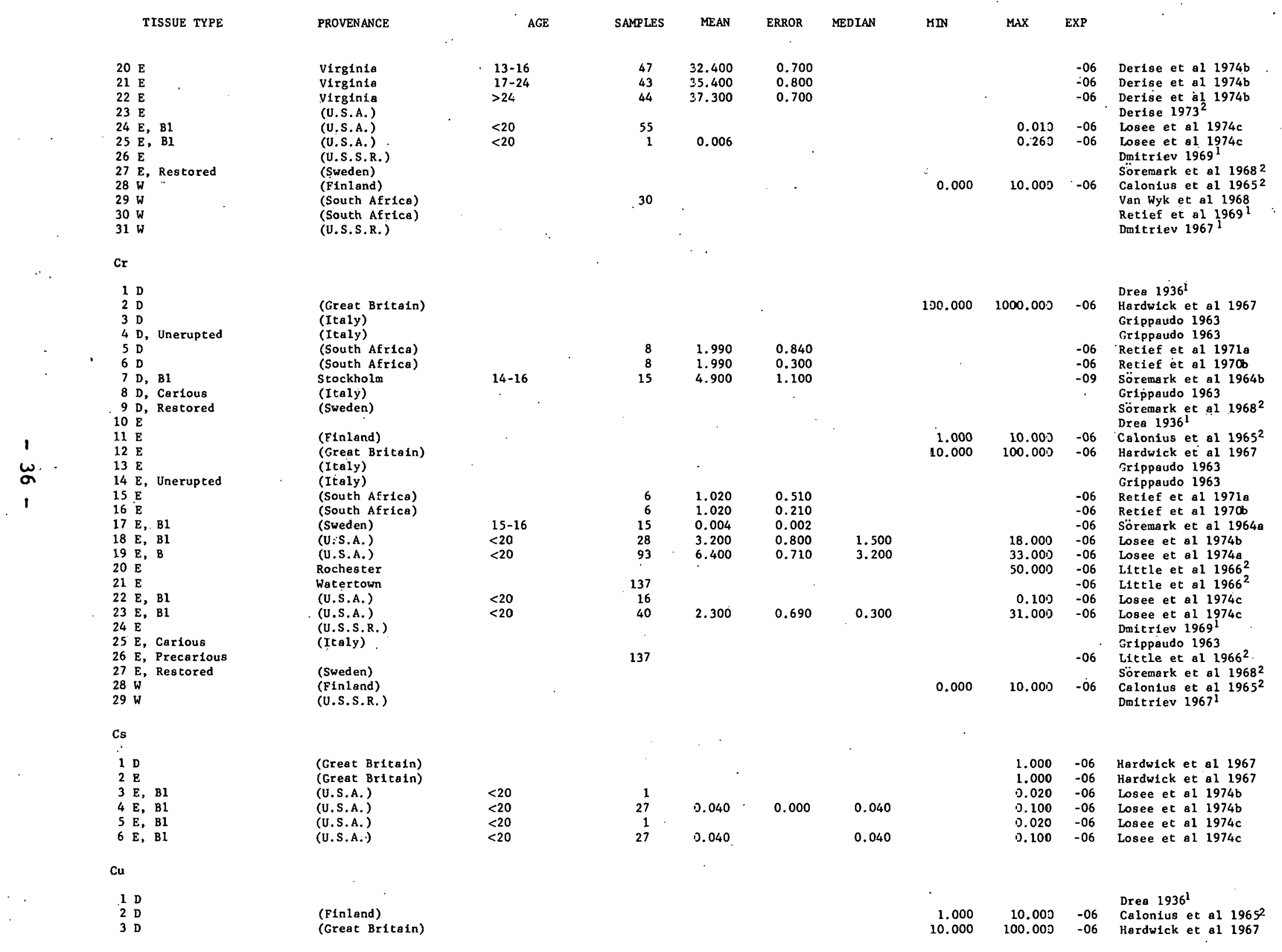




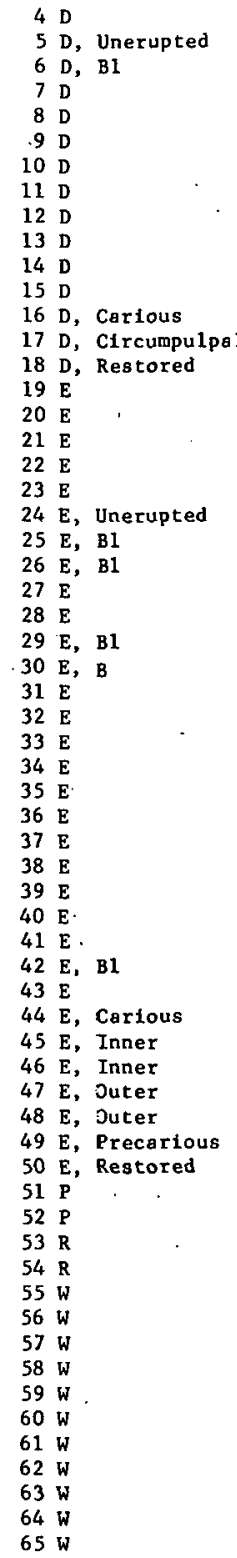

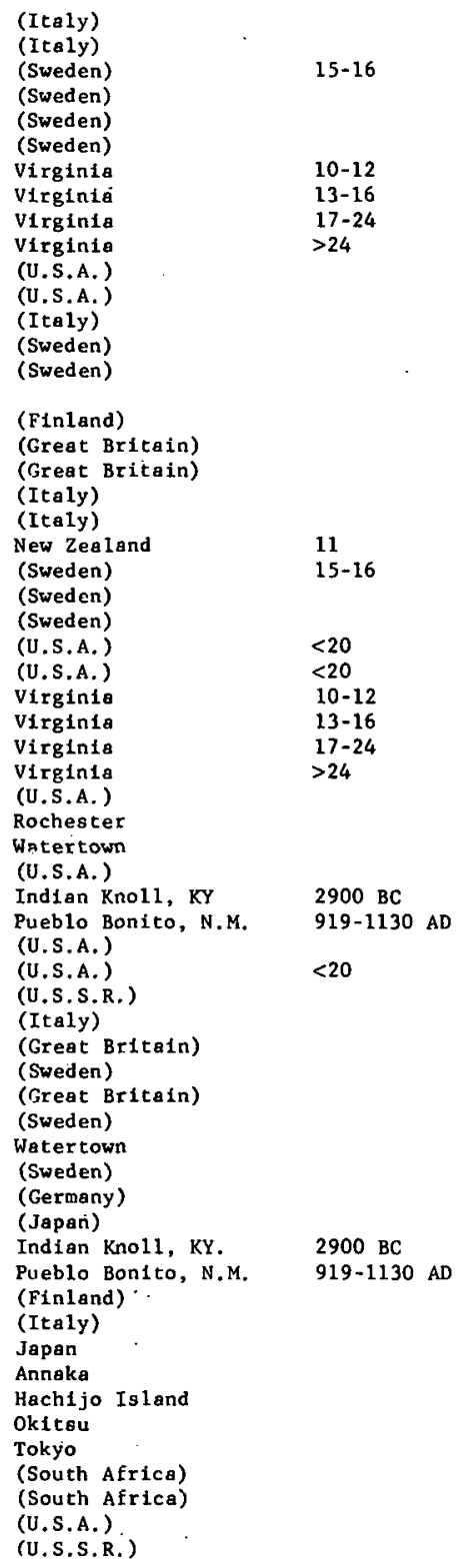

$\begin{array}{lll}15 & 0.210 & 0.100 \\ & & \\ 1 & & \\ 39 & 7.500 & 1.300 \\ 47 & 7.800 & 1.200 \\ 43 & 5.800 & 1.200 \\ 44 & 7.400 & 1.200 \\ & & \\ & & \\ 1 & 13.000 & \end{array}$

103

$10.110 \quad 7.840$

$\begin{array}{ll}36 & 0.170 \\ 15 & 0.260\end{array}$

0.020
0.110

$28 \quad 4.200$

$\begin{array}{ll}93 & 12.200 \\ 39 & 11.500 \\ 47 & 14.400\end{array}$

$\begin{array}{ll}47 & 14.400 \\ 43 & 10.100 \\ 44 & 11.700\end{array}$

33.000
$137 \quad 31.900$

40.000

$56 \quad 6.800$

$100 \quad 11.300$

1
$100 \quad 9.500$

$\begin{array}{rr}1 & 18.000 \\ 137 & 68.900\end{array}$

3.010

1.460
2.000

2.000
1.800
1.900

1.800
1.900
1.900

1.900

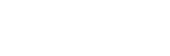

$\begin{array}{rrrr} & 53.000 & & \\ & 1.000 & & \\ & & & \\ & & & \\ 31 & 1.220 & 0.610 & 1.060 \\ 17 & 3.470 & 2.290 & 2.900 \\ 22 & 1.570 & 1.370 & 1.110 \\ 24 & 2.440 & 2.070 & 2.150 \\ 30 & & & \end{array}$

Grippaudo 1963 Gr1ppaudo 1963

6 Soremark et al 1962b Samsahl et al 1961a Samsah1 et al 1961b.

2.200 -06
-06
-06
-06 Derise et al 1974b Derise et al 1974b Derise $1973^{2}$ Asgar 1956 Gr1ppaudo 1963 Ah1berg et a1 1976 Soremark et a1 $1968^{2}$ Calontus et al $1965^{2}$ Calonice al $1967 c$ Herdw1ck et a1 1967 Grippaudo 1963 Grippaudo 1963

-06 Curzon et al $1975^{2}$ Soremark et al 1962a Samsahl et al 1961a Samsah1 et al 1961 Losee et al 1974b

Derise et al $1974 b$

Derise et al 1974b Derise et al 1974b Derise et al 1974b Der1se $1973^{2}$

Little et al $1966^{\circ}$ Det al $1966^{2}$ Brudevold et al $1955^{2}$ Steadman et al $1959^{2}$ Asgar 1956 . Losee et al $1974 \mathrm{c}$. Grippaudo 1963 Nixon et al 1962 Ahlberg et al 1976 Nixon et a.l $1962^{4}$
Ahlberg et al 1976 Ahlberg et al 1976
Little et al $1966^{2}$ Sortle et al al al $1968^{2}$

Higashi et al $1969^{1}$ Steadman et al $1959^{2}$ Stéadman et al $1959^{2}$ Calonius et al $1965^{2}$ Pleruccini $1949^{1}$ Kaneko et al 1974 Kaneko 1972 4 Kaneko 1972

Kaneko $1972^{4}$

Van Wyk et al $1968^{\circ}$ Van Wyk et al $1968^{\circ}$ Fields et al $1950^{1}$ Dmitriev $1967^{1}$ 


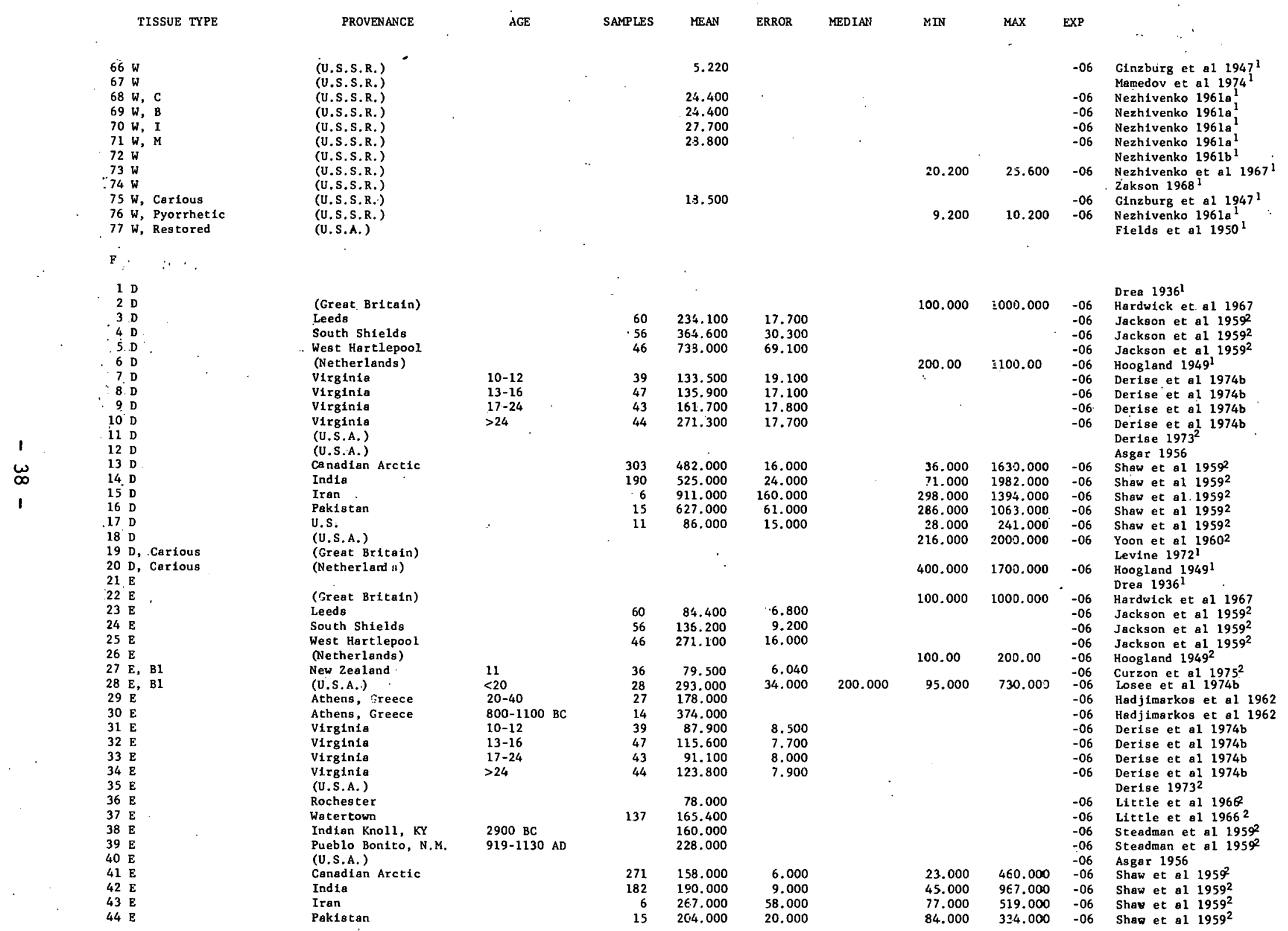




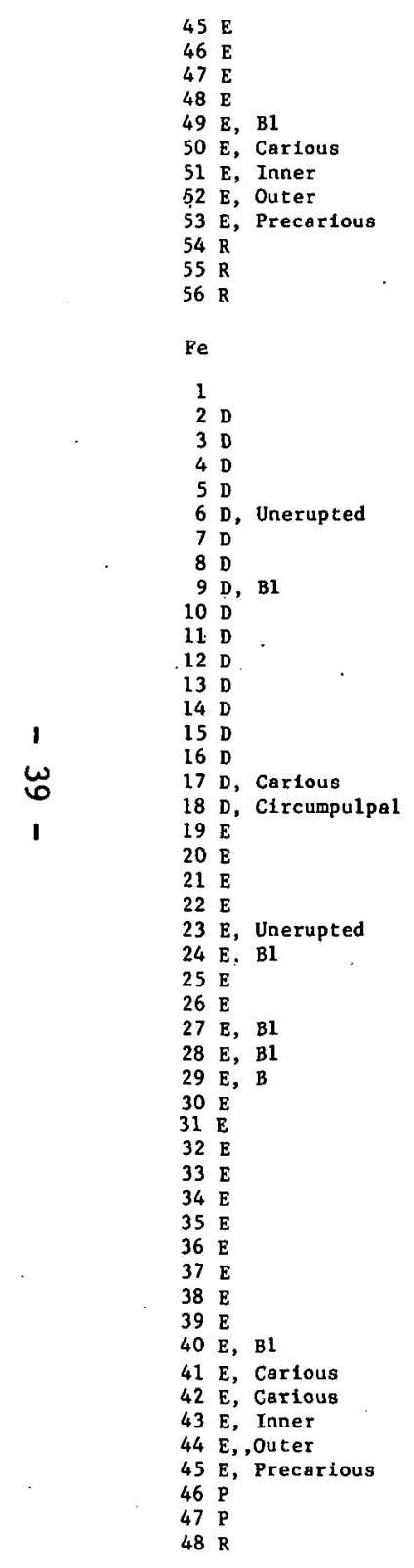

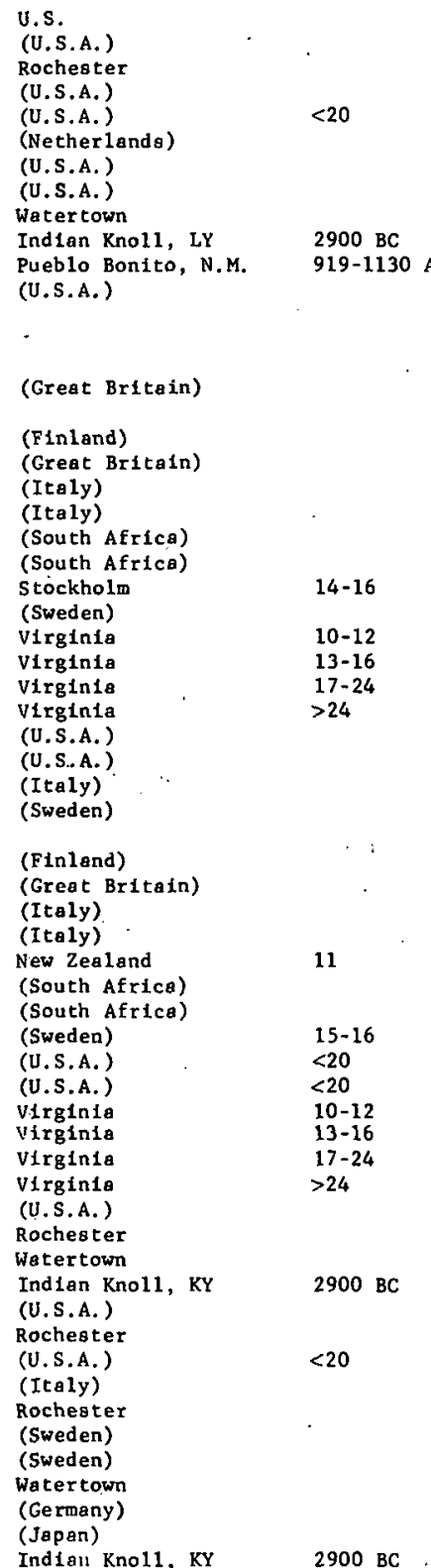
$12 \quad 54.000$
120.000
$56 \quad 248.000$
$137 \quad 777.600$ $\begin{array}{r}940.000 \\ \hline \quad 934.000\end{array}$

$\begin{array}{rrr}9 & 93.380 & 35.050 \\ 9 & 93.380 & 11.680 \\ 15 & 110.000 & 22.000 \\ 1 & 5.500 & \\ 39 & 42.100 & 1.700 \\ 47 & 43.000 & 1.500 \\ 43 & 46.000 & 1.600 \\ 44 & 40.100 & 1.600\end{array}$

$1 \quad 14.000$

$\begin{array}{lll}36 & 18.300 \quad 1.890\end{array}$

118.270

338.000

4.400

63.800

38.600

39.100

39.100
41.600

20.000

$137 \quad 22.900$

25.000
$56 \quad 9.500$

52.000

$\begin{array}{rr}1 & 9.000 \\ 1 & 48.000 \\ 137 & 22.000\end{array}$

$137 \quad 22.000$

80.000

050
.680

22.000

1.500
1.600
1.600

\subsection{650}

71.650
23.880

109.000
0.950

0.950
9.780
1.300

1.200
1.200

1.200

0.960

$$
80.000
$$

7.000

20.000

200.000

70.000

32.000

$33.000 \quad 1247.000$

$718.000 \cdot 9360.000$

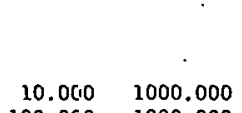

$\begin{array}{rr}10.0010 & 1000.000 \\ 100.000 & 1000.000\end{array}$

$$
-06
$$

.

1.000
100.000

10.000
1000.000

2.600
44.000

Indian Kno11, KY $2900 \mathrm{BC}$

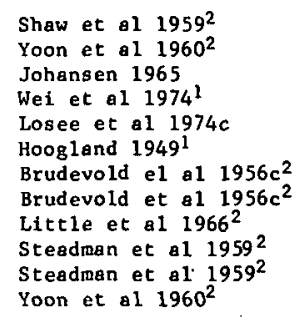


TISSUE TYPE

\section{$49 \mathrm{~W}$ \\ 51 \\ $52 \mathrm{~W}$ \\ $53 \mathrm{~W}$}

$\begin{array}{ll}1 & D \\ 2 & E\end{array}$

Ge

Hg

$1 \mathrm{D}$, Restored

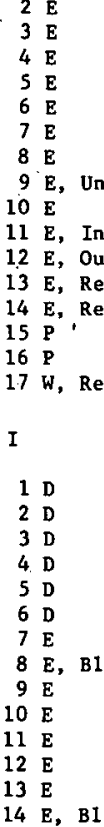

In

$\begin{array}{ll}1 & \mathrm{D} \\ 2 & \mathrm{E}\end{array}$
PROVENANCE

(EInland)

(Italy)
(South Africa)

(South Africa)

(U.S.S.R.)

(U.S.S.R.)

(Great Britain)
(Great Britain)

(Great Britain)

AGE

SAMPLE

MEAN

ERROR

MEDIAN

30

(Sweden)

Denmark

Denmark

Denmark

Denmark

(Great Britain)

(freat Britain)

(Grest Britain)

(Great Britain)

(Great Britain)

(Great Britain)

(Sweden)

(Germany)

(U.S.A.)

(Great Britain)

Virginia

Virginia

Virginia

(U.S.A.)

(Great Britain)

(U.S.A.)

Virginia
Virginia

Virginia
Virginia

Virginia

(U.S.A.)

(U.S.A.)

(Great Britain)

$1100-1400 \mathrm{AD}$
$1500-2300 \mathrm{BC}$
$1850 \mathrm{AD}$
$1970 \mathrm{AD}$
$400 \mathrm{BC}-200 \mathrm{AD}$
$800-1100 \mathrm{AD}$

9-35

$\begin{array}{lll}10-12 & 39 & 4.050 \\ 13-16 & 47 & 4.300 \\ 17-24 & 43 & 2.740 \\ >24 & 44 & 3.820 \\ & & \\ <20 & 28 & 0.036 \\ 10-12 & 39 & 4.660 \\ 13-i 6 & 47 & 6.860 \\ 17-24 & 43 & 5.270 \\ >24 & 44 & 6.610 \\ <20 & & \\ < & & 0.036\end{array}$

0.410
0.370
0.380
0.380

0.000
0.620
0.560
0.580
0.580

0.030

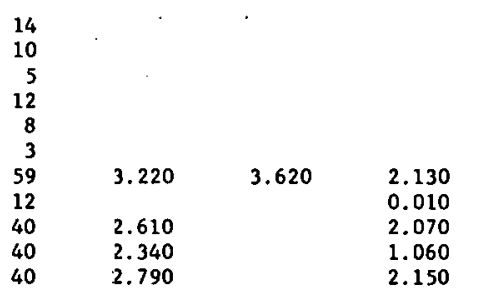

1.000
1.000

$\begin{array}{llll}10.000 & -06 & \text { Hardwick et a1 } 1967\end{array}$

$\begin{array}{lllll}1.000 & 10.000 & -06 & \text { Hardwick et a1 } & 1967 \\ 1.000 & 10.000 & -06 & \text { Hardwick et a 1 } & 1967\end{array}$

$\begin{array}{rrr}0.046 & 1.880 & -06 \\ 0.001 & 0.256 & -06 \\ 0.042 & 0.267 & -06 \\ 0.005 & 1.070 & -06 \\ 0.001 & 0.987 & -06 \\ 0.047 & 0.184 & -06 \\ 0.140 & 18.100 & -06 \\ & 3.460 & -06 \\ & & -06 \\ & & -06 \\ & & -06\end{array}$

Söremark et al 1968 Rasmusaen 1974 Rasmassen 1974 Rasmussen 1974 Rasmnssen 1974 Rasmassen 1974 Rasmassen 1974 N:xon et al 1967c Nixon et al $1965^{4}$ N1xon et a1 $1965^{4}$ Nixon et al $1965^{4}$ N1xon et a1 $1965^{4}$ Sörenark et al $1968^{2}$ Jost $1934^{1}$ Higashi et al $1969^{1}$
Fields et a1 $1950^{1}$

0.010

0.030

0.010

$0.07 \mathrm{C}$

1.000

-06 Hardoick et al 1967

-06 Derlse et al 1974b

Derlae et al 1974b

Derise et al 1974b Derise $1973^{2}$

Hardulck et al 1967 Losee et al 1974b Derise et al 1974b Derise et al 1974b Derise et al 1974b Derise et al $1974 b$

Losee et à 1974 c 
K

$\begin{array}{ll}1 \mathrm{D} & \\ 2 \mathrm{D} & \text { (Finland) } \\ 3 & \mathrm{D}\end{array}$

Virgenta

Virginia

Virginia

Virginia
(U.S.A.)

(Sweden)

(Finland)

(Great Britain)

Vis.A.)

vising

Virginie

Virginia

(U.S.'A.)

(U.S.A.)

(U.S.A.)

(Sweden)

(Sweden)

(Finland)

(Germany)

(Germany)

27 W, Deciduou

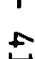

$\stackrel{5}{1}$

$3 \mathrm{D}$, Unerupted, $\mathrm{M} 3$

4 B

$5 \mathrm{E}, \mathrm{M3}$.

$6 \mathrm{E}, \mathrm{Bl}$
$7 \mathrm{E}, \mathrm{Bl}$

L1

$\begin{array}{ll}1 & \mathrm{D} \\ 2 & \mathrm{E}\end{array}$

$3 \mathrm{E}, \mathrm{B} 1$

$4 \mathrm{E}, \mathrm{B1}$

$5 \mathrm{E}, \mathrm{BI}$

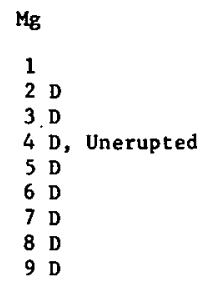

Britatn

(Norway)

(Gréat Britain)

(Norway)

(U.S.A.)

(U.S.S.R.)

New Zealand

(U.S.A.)

(U.S.A.)

(France)

(U.S.A.)

(Italy)

(South Africa)

(South Africa)

Virginia

Virginia
Virginia
$10-12$
$13-16$
$17-24$
$>24$

$\begin{array}{rll}1 & 0.060 & \\ 39 & 0.017 & 0.001 \\ 47 & 0.017 & 0.001 \\ 43 & 0.017 & 0.001 \\ 44 & 0.020 & 0.001 \\ 1 & 0.160 & \end{array}$

$<20$

$10-12$
$13-16$

$17-24$

$<20$

$10-12$
$13-16$
$17-24$

$21-22$
25
$21-72$
$<20$
$<20$

11
$<20$
$<20$

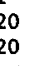

1.100
0.130
0.120

$\begin{array}{rrrr}28 . & 401.000 & 31.000 & 370.000 \\ 39 & 0.026 & 0.001 & \\ 47 & 0.025 & 0.001 & \\ 45 & 0.026 & 0.001 & \\ 44 & 0.031 & 0.001 & \\ & 0.300 & & \\ 56 & 458.000 & 23.000 & 490.000 \\ 1 & 0.070 & & \\ 1 & 0.220 & & \\ & 0.330 & & \\ & 0.340 & & \\ & 0.350 & & \end{array}$

$\begin{array}{lll}3 & 12.030 & 2.500\end{array}$

$\begin{array}{lll}4 & 5.400 & 0.850\end{array}$

0.004

0.930
1.400

0.230

$180.030 \quad 1000.000$

$1.030 \quad 1000.000$

$$
1000.000
$$

$\begin{array}{crr}7.200 & 1.000 & -06 \\ & 15.300 & -09 \\ & & -09 \\ 4.100 & 1.000 & -06 \\ & 7.700 & -09 \\ & 0.020 & -06 \\ & 0.120 & -06\end{array}$

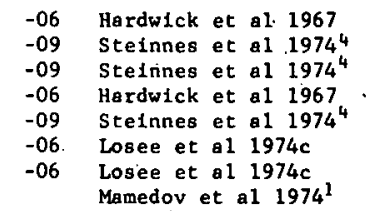

Drea $1936^{1}$

Drea 1936

Curzon et al $1975^{\circ}$

Losee et a1 1974b

Desgrez $1927^{1}$

Pleruccini et al $1949^{1}$

Bes1c et al $1969^{4}$ Drea 1936

Grippaudo 1963

Retief et a1 1970

Retief et al $1971 \mathrm{a}$

Derise et al 1974

Derise et al 1974a
Derise et a1 1974a 
TISSUE TYPE

PROVENANCE

$10 \mathrm{D}$

$\begin{array}{ll}11 & \mathrm{D} \\ 12 & \mathrm{D} \\ 13 & \mathrm{D}\end{array}$

$13 \mathrm{D}$

is D, Carious

$16 \mathrm{E}$

$18 \mathrm{E}$, Unerupted

19

$20 E$
$21 E ;$
$E$

$22 \mathrm{E}$.

$23 \mathrm{E}$
$24 \mathrm{E}$
$25 \mathrm{E}$

$26 \mathrm{E}$

$27 \mathrm{E}$

$29 \mathrm{E}$

$30 \mathrm{E}$, Carious

$31 \mathrm{E}$, Carious

$33 \mathrm{~W}$, Deciduous

$34 \mathrm{~W}, \mathrm{M3}$

$36 \mathrm{~W}$.

$37 \mathrm{~W}$

$38 \mathrm{~W}$

$40 \mathrm{~W}$

$41 \mathrm{~W}$, Carfous

42 w, Pyorrhetic

$43 \mathrm{w}$, Restored

Mn

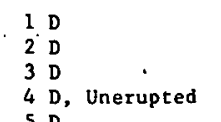

D, Unerupted

$6 \mathrm{D}$
$7 \mathrm{D}, \mathrm{B}$

8 D, Unerupted,

$9 \mathrm{D}$

$10 \mathrm{D}$

$11 \mathrm{D}$

$13 \mathrm{D}$

$14 \mathrm{D}$

$15 \mathrm{D}$
$16 \mathrm{D}$

$17 \mathrm{D}$, Unerupted, Femal

$18 \mathrm{D}$, Unerupted, Male

$19 \mathrm{D}$, Male

$20 \mathrm{D}$, Female

$22 \mathrm{D}$, Circumpulpal
Virginia

(U.S.A.)

U.S.

(U.S.A.)

(Italy)

(Italy)

(South Africa)

(South Africa)

v1rginia

Virginis

Virginia

Virginia

(U.S.A.)

Greece

Rochester

(Italy)

(Germany)

(Germany)

(U.S.A.)

(U.S.A.)

(U.S.A.)

(U.S.S.R.)

(II.S.A.)

(Great Britain)

(Ita1;)

(South Africa)

(South Africa)

(Sweden)

(Sweden)

(Sweden)

(Sweden)

Virginia

Virginia

(U.S.A.)

U.S. East Cosst

J.s. East Coast

U.S. East Coast

(Italy)

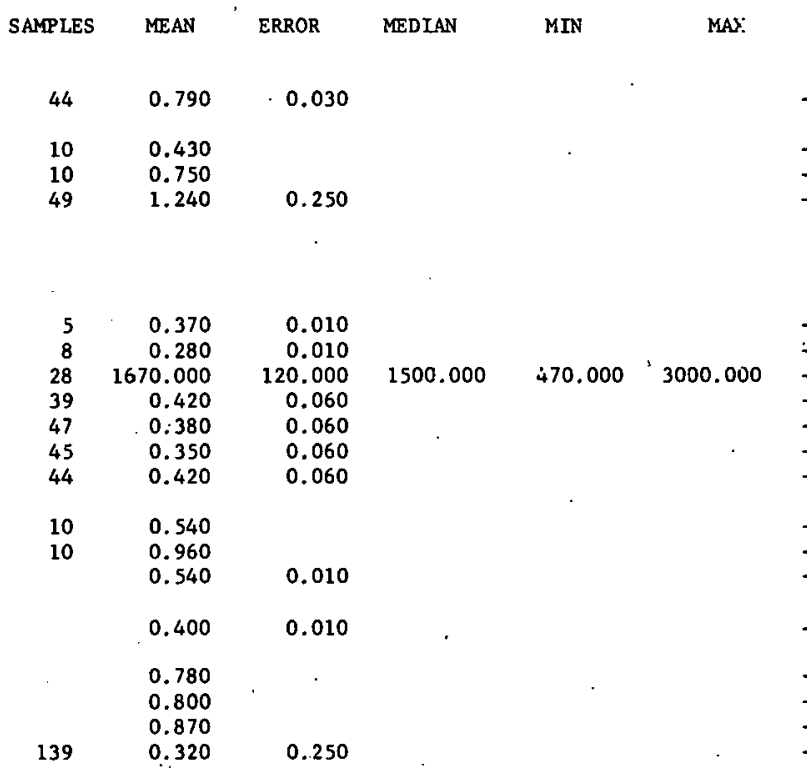

139

0.320

0.250

$15-16$
$14-25$

$\begin{array}{rll}5 & 0.630 & 0.050 \\ 5 & 0.630 & 0.020 \\ 15 & 0.190 & 0.060 \\ 10 & 0.560 & 0.310 \\ & & \\ 1 & & \\ 39 & 6.200 & 0.160 \\ 47 & 5.270 & 0.140 \\ 43 & 5.580 & 0.150 \\ 44 & 5.520 & 0.150 \\ 17 & 0.310 & 0.020 \\ 9 & 0.260 & 0.040 \\ 27 & 0.240 & 0.020 \\ 25 & 0.250 & 0.020\end{array}$

$10.000 \quad 100.050$

EXP

02 Derise et al 1974a

- Derise $1973^{2}$

Asgar 1956

02 Tefft et al 1941

Grippaudo 1963

Drea $1936^{1}$

Grippaudo 1963

Grippaudo 1963

Retief et al 1970a

Losee et al 1974b

Derise et al 1974 a

-Derise et a1 1974a

Derise et al 1974a

Derise et a1 1974a

Derise $1973^{2}$

Asgar 1956

Asgar 1956

Johansen 1965

Grippaudo 1963

Johansen 1965

Gasamann 1

Crowell, Jr. et al 1934

Fields et al 1950

Lundstrom 1931

Damedov et al 197

Kaushansky 1932

Keushansky 1932

Drea 1936

Hardw1ck et al 1967 Grippaudo 1963

Retlef et al 197

Rẹtlef et al 1971b

Soremark et al 1962b

Lundberg et al 1965b Samsah1 et al 1961a Samsahl et al 1961b

5.000 Ahlberg et al 1976

Derise et al 1974b

Derise et al 1974b

Derise et a1 1974b

Derise $1973^{2}$

Battistone et al 1964

Battistone et al $1967^{4}$

Battistone et al 19674.

Battistone et al $1967^{4}$

Grippsudo 1963

Ahroerg et al 1976 


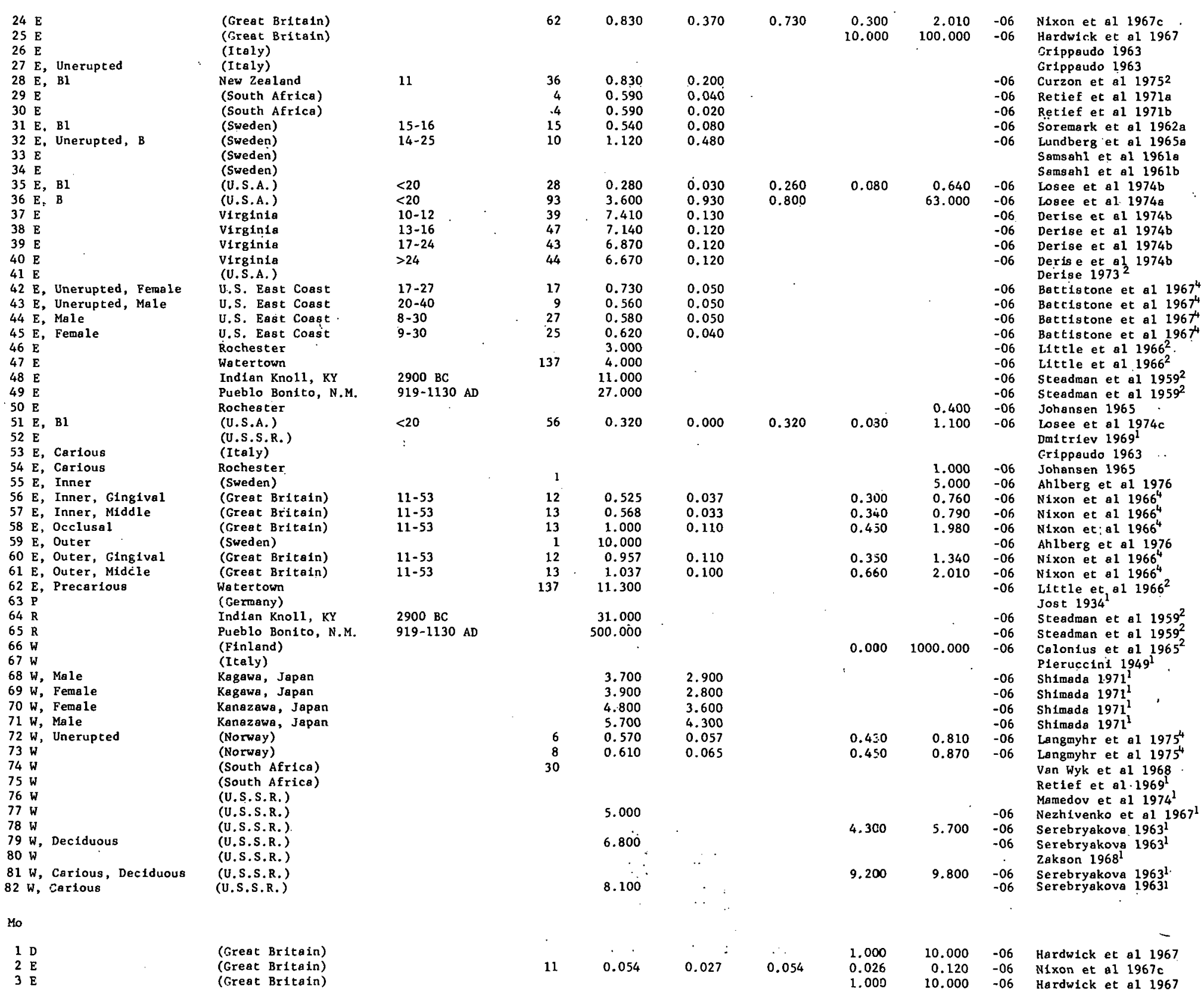




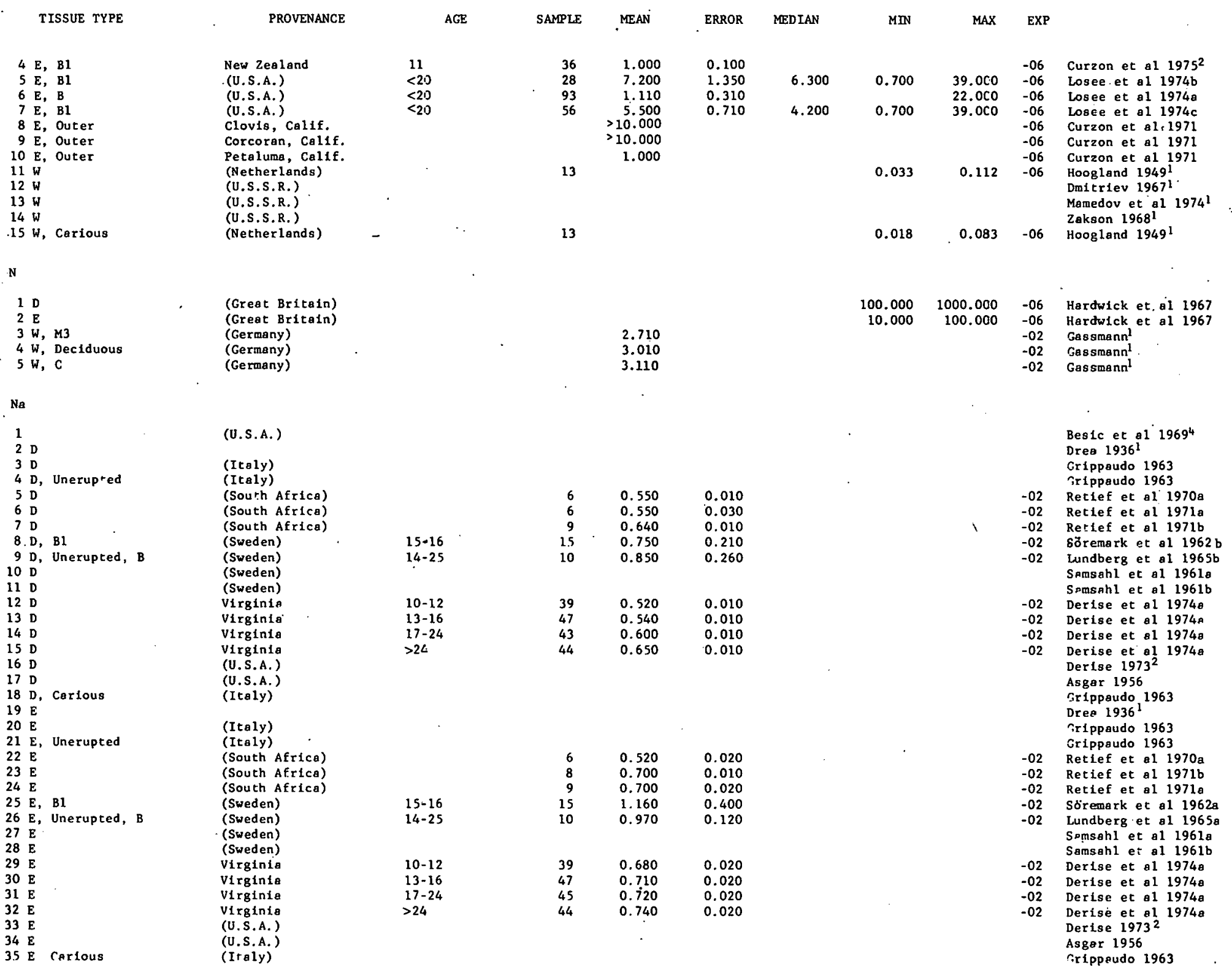




$\begin{array}{ll}36 \mathrm{P} & \\ 37 \mathrm{~W}, \text { Deciduous } & \text { (Jepan) } \\ 38 \mathrm{~W} . & \text { (Germany) } \\ 39 \mathrm{~W} . \mathrm{M} 3 & \text { (Germeny) } \\ 40 \mathrm{~W} & \text { (ermany) } \\ 41 \mathrm{~W} & \text { (Italy) } \\ 42 \mathrm{~W} & \text { (South Africa) } \\ 43 \mathrm{~W} & \text { (South Africa) } \\ 44 \mathrm{~W} & \text { (U.S.A.) } \\ 45 \mathrm{~W}, \text { Rrstored } & \text { (U.S.S.R.) } \\ & \text { (U.S.A.) }\end{array}$

$\mathrm{Nb}$

$\begin{array}{ll}I & E \\ 2 & E, B 1\end{array}$

$\begin{array}{lll}2 & \mathrm{E}, \mathrm{Bl} \\ 3 \mathrm{E}, & \mathrm{BI} \\ 4 & \mathrm{E}, \mathrm{Bl}\end{array}$

$5 \mathrm{E}, \mathrm{BI}$

na

$\begin{array}{lll}1 & E, & B 1 \\ 2 & E & B 1\end{array}$

$3 \mathrm{E}, \mathrm{Bl}$

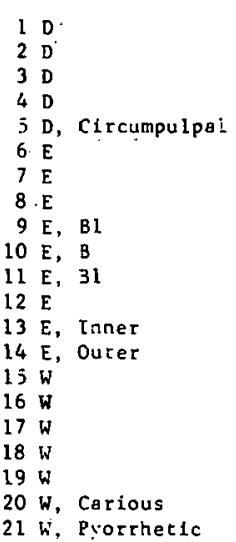

(Great Britain)

(Itely)

(Sweden)

(Sweden)

(cinland)

(Italiv)

New Zealand

(U.S.A.)

(U.S.A.)
(U.S.S.R.)

(Sweden)

(Sweden)

(Finland)

(U.S.S.R.)

(U.

(U.S.S.R.)

(U.S.S.R.)

(L.S.S.R.)

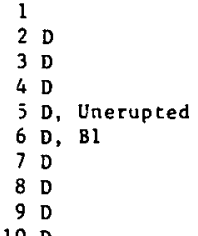

(C.S.A.)

(Finland)

(Italy)

(Italy)

(Sweden)

(Sweden)

Irginia
0.540

0.610
0.670

30

-02
-02
-02

Higash1 et al $1969^{1}$

Gassmann

Pierucrini $1949^{1}$ $y=n$ Wyk et al 1968 Rerlef et al 1969 Fields et al $1950^{\circ}$ Mamedov et al 1974

$\begin{array}{llll}36 & 0.600 & 0.050 & \\ 28 & 0.280 & 0.030 & 0.240\end{array}$

0.760

0.100
0.760 $\begin{array}{ll}-06 & \text { Hardwick et } 811967 \\ -06 & \text { Curzon et o1 } 1975^{2}\end{array}$ Losee et al $1974 \mathrm{c}$

0.240

0.020

$0.090 \quad-06$ Losee et al 1974b

-06 Losee et al 1974b

$\begin{array}{llll}0.020 & -06 & \text { Losee et } 81 & 1974 c \\ 0.090 & -06 & \text { Losee et al } 1974 \text { c }\end{array}$

1.001
10.000

10.000
100.000

2.500

10.000

1.000

100.000

11
$<20$
$<20$

$\begin{array}{lll}36 & 0.210 & 0.050 \\ 93 & 1.570 & 0.270\end{array}$

0.050
0.270
0.140

0.410

0.110

13.000

3.000

2.500

4.500

0.000

1.350

0.250

0.160

0.220

10.000

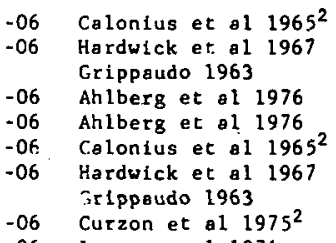

-06 Curzon et al $1975^{2}$

Losee et al 1974a

Losee et al 1974b

Dmicriev 1969'

Ahlberg et al 1976

Ahlbers et al 1976
Calonius et al $1965^{2}$

Calonius et al $1965^{2}$

-06 Nezhivenko et a1 19671

-06 "ihm $1964^{1}$

-06 :1hm 1964

"ihm $1964^{1}$

Dreo $1936^{2}$

-02 Calonius et al $1965^{2}$ Srippaudo 1963.

Trippaudo 1963
Söremark et al 1962b

Söremark et al 1962b
Samsahl et al 1961a

Ahlberg et al 1976

$10-12$

$\begin{array}{rr}1 & 19.000 \\ 39 & 13.400\end{array}$

0.100 


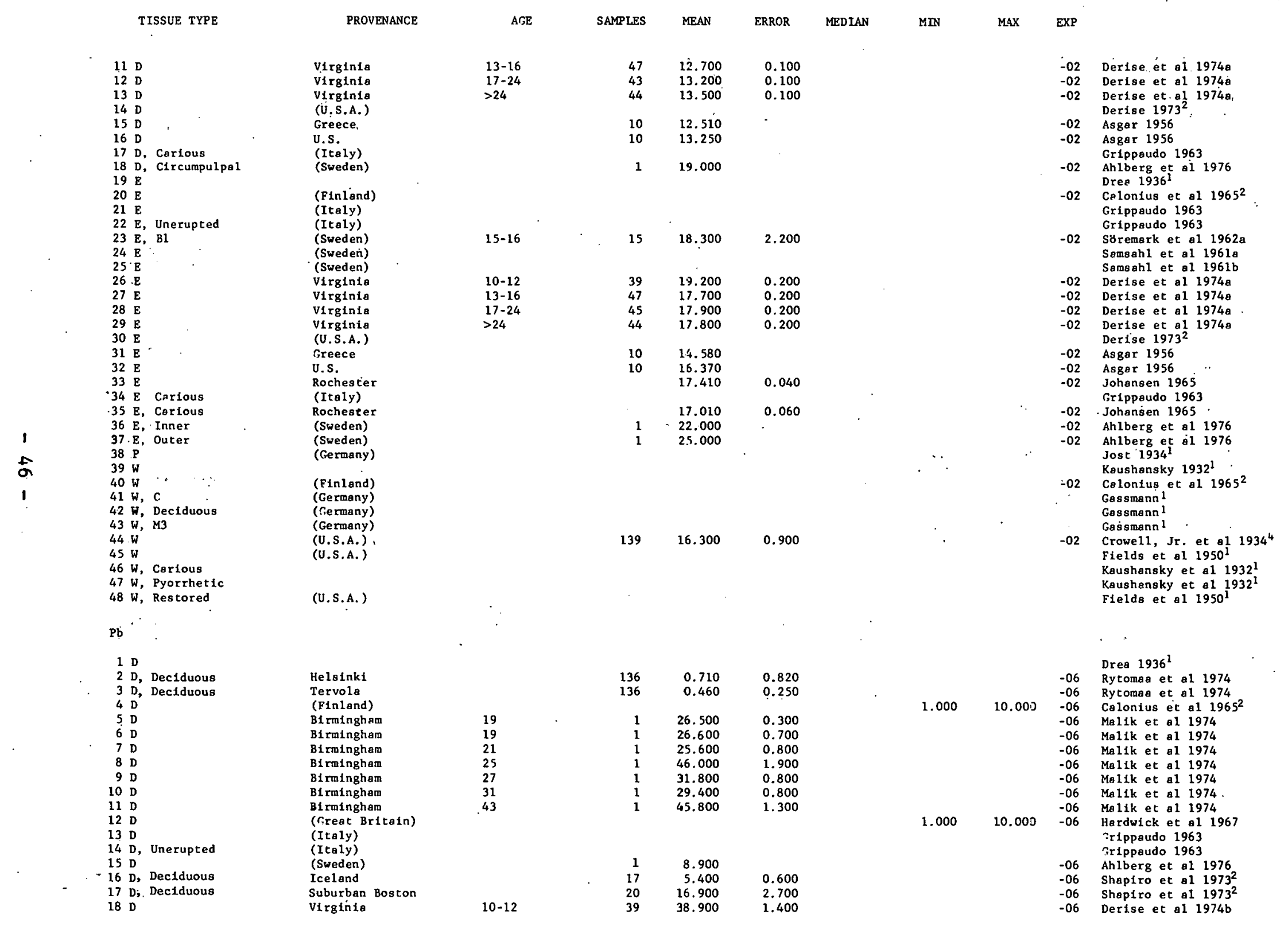




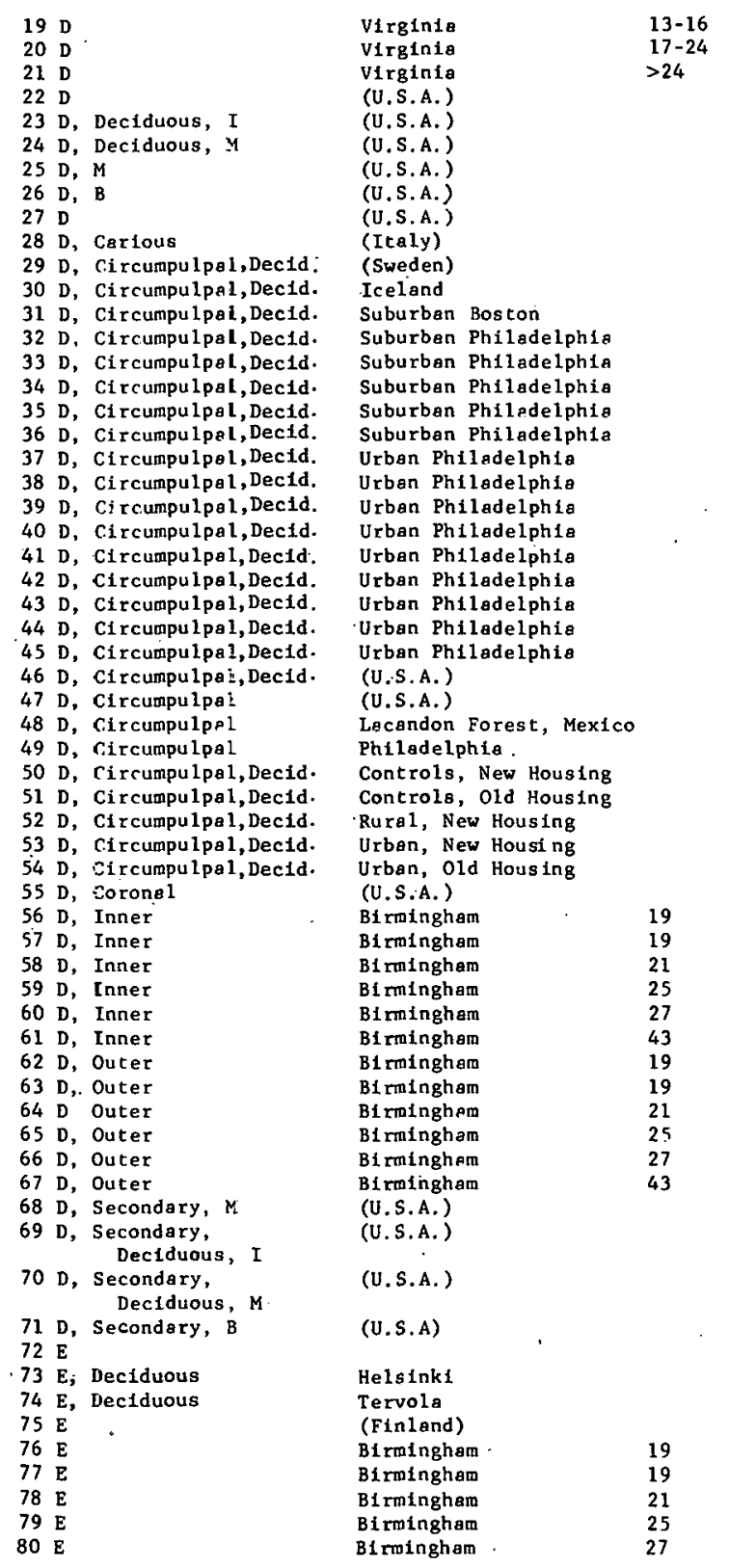

$\begin{array}{rrr}47 & 42.200 & 1.200 \\ 43 & 40.700 & 1.300 \\ 44 & 51.500 & 1.300 \\ & & \\ 3 & 23.700 & 1.800 \\ 4 & 28.800 & 8.100 \\ 4 & 49.500 & 12.400 \\ 3 & 56.300 & 21.100 \\ & & \\ 1 & 29.000 & \\ 17 & 35.500 & 7.400 \\ 20 & 84.400 & 12.700 \\ 69 & 37.000 & 2.600 \\ 59 & 40.000 & 2.900 \\ 114 & 42.000 & 2.800 \\ 76 & 46.000 & 2.800 \\ 62 & 48.000 & 3.600 \\ 40 & 66.000 & 5.600 \\ 1 & 92.000 & 7.800 \\ 34 & 120.000 & 21.200 \\ 19 & 131.000 & 24.700 \\ 71 & 136.000 & 15.600 \\ 29 & 169.000 & 27.500 \\ 33 & 188.000 & 30.200 \\ 58 & 191.000 & 19.700 \\ 46 & 208.000 & 29.000 \\ & & \\ 36 & 4.300 & 6.800 \\ 36 & 92.300 & 64.800 \\ 35 & 97.800 & 9.570 \\ 22 & 87.800 \\ 10 & 171.000 & 28.910 \\ 4 & 19.000 & 5.910 \\ 10 & 153.900 & 52.820 \\ 22 & 212.900 & 39.750 \\ 1 & 25.300 & 0.600 \\ 1 & 26.700 & 1.000 \\ 1 & 25.000 & 1.000 \\ 1 & 1.900 \\ 1 & 38.500 & 1.000 \\ 1 & 27.400 & 1.800 \\ 1 & 41.000 & 1.000 \\ 1 & 26.300 & 1.00 \\ 1 & 26.800 & 0.830 \\ 1 & 27.800 & 1.400 \\ 1 & 49.800 & 2.000 \\ 1 & 32.300 & 1.200 \\ 1 & 50.600 & 1.900 \\ 3 & 142.000 & 17.100 \\ 3 & 143.300 & 52.500 \\ 4 & 222.300 & 82.200 \\ 4 & & \\ 3 & 242.700 & 42.100 \\ 136 & 69.900 & 68.400 \\ 136 & 81.400 & 44.600 \\ & & \\ 1 & 28.300 & 0.900 \\ 1 & 30.200 & 1.200 \\ 1 & 24.500 & 1.000 \\ 1 & 53.500 & 2.100 \\ 1 & 41.700 & 0.900\end{array}$

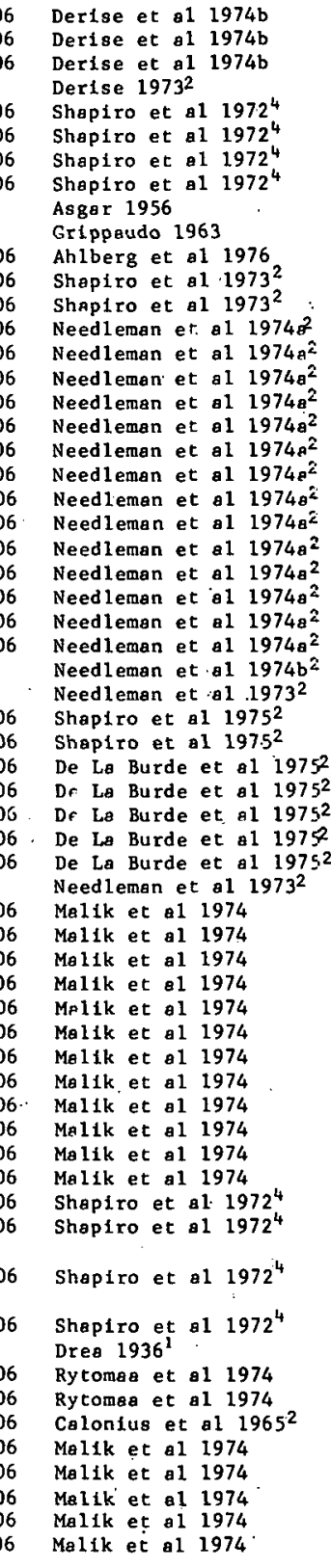




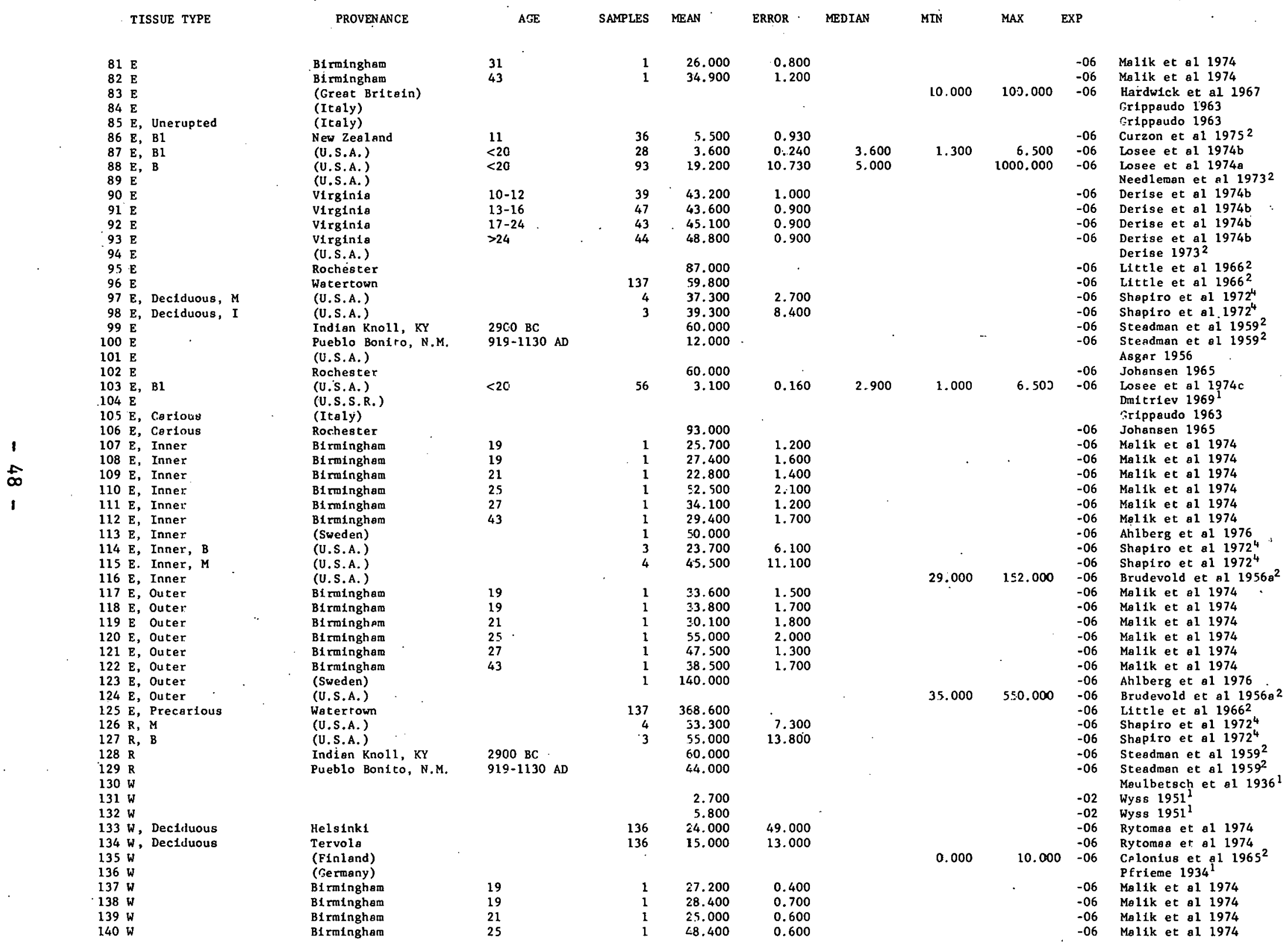




\begin{tabular}{|c|c|c|c|c|c|c|c|c|c|c|c|c|}
\hline $141 \mathrm{w}$ & & B1rminghan & & 27 & 1 & 36.600 & 0.600 & & & & -06 & Molik et a1 1974 \\
\hline $142 \mathrm{w}$ & & B1rmimghan & & 31 & 1 & 27.300 & 0.600 & & & & -06 & Ma1ik et al 1974 \\
\hline $143 \mathrm{w}$ & & Blrmfnghat & & 43 & 1 & 40.400 & 0.900 & & & & -06 & Malik et al 1974 \\
\hline $144 \mathrm{~W}$ & & Bliminghan & & 46 & 1 & 42.100 & 0.600 & & & & -06 & Ma1ik et al 1974 \\
\hline 145 i & & Birminghan & & 52 & 1 & 51.500 & 0.700 & & & & -06 & Malik et al 1974 \\
\hline $146 \mathrm{w}$ & & Birminghar & & 57 & $\mathrm{i}$ & 52.700 & 0.800 & & & & -06 & Malik et al 1974 \\
\hline $147 \mathrm{~W}$ & & (Great Bri & itgin) & & & & & & & & & Campbe11 et al $1950^{1}$ \\
\hline $148 \mathrm{~W}$ & , Deciduous, MI & Rural Co. & Fermanagh & 10 & 11 & $6.300^{3}$ & 2.420 & & & & -06 & Stewert 1974 \\
\hline $149 \mathrm{w}$ & , Deciduous, M1 & Rural Co. & Fermanagh & 3 & 2 & $0.800^{3}$ & 1.970 & & & & -06 & Stewart 1974 \\
\hline $150 \mathrm{w}$ & , Dec1duous, M1 & Rural Co. & Fermanagh & 4 & 5 & $2.930^{3}$ & 0.750 & & & & -06 & Stewart 1974 \\
\hline $151 \mathrm{w}$ & , Declduous, M1 & Rurel Co. & Fermanagh & 5 & 14 & $3.010^{3}$ & 2.610 & & & & -06 & Stewart 1974 \\
\hline $152 \mathrm{w}$ & , Deciduous, M1 & Rural Co. & Fermanagh & 6 & 11 & $4.120^{3}$ & 1.950 & & & & .06 & Stewart 1974 \\
\hline $153 \mathrm{w}$ & , Deciduous, MI & Rural Co. & Fermanagh & 7 & 13 & $3.050^{3}$ & 2.990 & & & & -06 & Stewart 1974 \\
\hline $154 \mathrm{k}$ & , Dec1duous, MI & Rural Co. & Fermanagh & 8 & 8 & $4.440^{3}$ & 1.670 & & & & -06 & Stewart 1974 \\
\hline 155 น & , Dectduous, MI & Rural Co. & Fermanagh & 9 & 7 & $6.300^{3}$ & 2.150 & & & & -06 & Stewart 1974 \\
\hline $156 \mathrm{w}$ & , DecIduous, MI & Suburban & Bel fast & 10 & 5 & $14.050^{3}$ & 6.400 & & & & -06 & Stewart 1974 \\
\hline $157 \mathrm{~W}$ & , Deciduous, M1 & Suburban & Bel fost & 3 & 12 & $3.780^{3}$ & 1.560 & & & & -06 & Stewart 1974 \\
\hline $158 \mathrm{k}$ & , Dec1duous, MI & Suburban & $\begin{array}{l}\text { Bel fast } \\
\text { Bes }\end{array}$ & 4 & 18 & $4.350^{3}$ & 1.540 & & & & -06 & Stewart 1974 \\
\hline $159 \mathrm{k}$ & , Deciduous, MI & Suburban & Belfast & 5 & 16 & $5.800^{3}$ & 1.940 & & & & -06 & Stewart 1974 \\
\hline $160 \mathrm{w}$ & , Dec1duous, M1 & Suburban & Belfast & 6 & 13 & $5.340^{3}$ & 1.910 & & & & -06 & Stewart 1974 \\
\hline $161 \mathrm{~W}$ & Declduous, M1 & Suburban $B$ & Bel fast & 7 & 7 & $5.660^{3}$ & 3.680 & & & & -06 & Stewart 1974 \\
\hline $162 \mathrm{~W}$ & , Deciduous, MI & Suburban $B$ & Belfast & 8 & 12 & $8.440^{3}$ & 4.200 & & & & -06 & Stewart 1974 \\
\hline $163 \mathrm{w}$ & Deciduous, MI & Suburban B & Belfast & 9 & 8 & $7.990^{3}$ & 2.200 & & - & & -06 & Stewart 1974 \\
\hline $164 \mathrm{~W}$ & Decíduous, Ml & Urban Belf & fast & 10 & 7 & $13.440^{3}$ & 4.910 & & 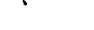 & & -06 & Stewart 1974 \\
\hline $165 \mathrm{~W}$ & Dec1duous, Ml & Urbon Belf & fast & 3 & 15 & $5.470^{3}$ & 2.370 & & & & -06 & Stewart 1974 \\
\hline $166 \mathrm{~W}$ & Deciduous, MI & Urban Belf & fast & 4 & 32 & $5.670^{3}$ & 2.170 & & & & -06 & Stewart 1974 \\
\hline $167 \mathrm{~W}$ & Declduous, Ml & Urban Belf & fast & 5 & 24 & $6.440^{3}$ & 2.230 & & & & -06 & Stewart 1974 \\
\hline $168 \mathrm{~W}$ & Deciduous, MI & Urben Belf & fast & 6 & 23 & $7.980^{3}$ & 4.000 & & & & -06 & Stewart $1974^{\circ}$ \\
\hline $169 \mathrm{w}$ & Dec1duous, MI & Urban Belf & fast & 7 & 19 & $7.870^{3}$ & 5.420 & & & & -06 & Stewart 1974 \\
\hline $170 \mathrm{~W}$ & Deciduous, MI & Urben Belf & fas r & 8 & 1.5 & $9.290^{3}$ & 4.650 & & & & $-06^{\circ}$ & Stewart 1974. \\
\hline $171 \mathrm{~W}$ & Dec1duous, MI & Urban Belf & fast & .9 & 13 & $10.120^{3}$ & 5.430 & & & & -06 & Stewart 1974 \\
\hline $172 \mathrm{~W}$ & & (Italy). & - & & & & & & & & & Pleruccini $1949^{1}$ \\
\hline $173 \mathrm{~W}$ & & Japan & & & & & & & & & & Kaneko et a1 1974 \\
\hline $174 \mathrm{~W}$ & & Annaka & & & 34 & 8.380 & 7.130 & 6.460 & 1.370 & 31.150 & -06 & Kaneko 1972 \\
\hline $175 \mathrm{~W}$ & & Hachijo Is & sland & & 17 & 15.670 & 18.430 & 9.160 & 1.120 & 64.670 & -06 & Kaneko 1972 \\
\hline $176 \mathrm{w}$ & & Okitsu & & & 19 & 5.630 & 3.510 & 4.480 & 1.500 & 13.270 & -06 & Kaneko 1972 \\
\hline $177 \mathrm{~W}$ & & Tokyo & & & 23 & 15.170 & 11.290 & 12.240 & 3.930 & 46.930 & -06 & Kaneko 1972 \\
\hline $178 \mathrm{~W}$ & & (Norway) & & & 8 & 2.300 & 0.405 & & 1.100 & 4.900 & -06 & Langmyhr et al $1974^{4}$ \\
\hline $179 \mathrm{~W}$ & Unerupted & (Norway) & & & 6 & 2.517 & 0.828 & & 1.100 & 6.400 & -06 & Lengmyhr et al $1974^{4}$ \\
\hline $180 \mathrm{~W}$ & 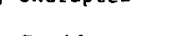 & South Caro & olina & & 18 & 92.400 & 41.900 & & 33.000 & 167.900 & -06 & Habercam et al 1974 \\
\hline $181 \mathrm{w}$ & , Dec1duous & Suburban $P$ & PhIladelphia & & 40 & 11.100 & 14.800 & & & & -06 & Needleman et al 1972 \\
\hline $\begin{array}{l}182 \mathrm{~W} \\
183 \mathrm{~W}\end{array}$ & Dectduious & $\begin{array}{l}\text { Urban Phil } \\
\text { (U.S.A.) }\end{array}$ & ladelphia & & 69 & 51.100 & 109.000 & & & & -06 & $\begin{array}{l}\text { Needleman et al } 1972 \\
\text { Needleman et al } 1974 \mathrm{~b}^{2}\end{array}$ \\
\hline $184 \mathrm{~W}$ & & (U.S.A.) & & $<10$ & 16 & 17.000 & 1.000 & & & & -06 & Strehlow et al 1969 \\
\hline $185 \mathrm{~W}$ & & (U.S.A.) & & $10-20$ & 37 & 22.000 & 7.000 & & & & -06 & Strehlow et al 1969 \\
\hline $186 \mathrm{~W}$ & & (U.S.A.) & & $20-30$ & 37 & 36.000 & 20.000 & & & & -06 & Strehlow et al 1969 \\
\hline $187 \mathrm{~W}$ & & (U.S.A.) & & $30-40$ & 30 & 59.000 & 10.000 & & & & -06 & Strehlow et al 1969 \\
\hline $188 \mathrm{~W}$ & & (U.S.A.) & & $40-50$ & 38 & 72.000 & 13.000 & & & & -06 & Strehlow et al 1969 \\
\hline $189 \mathrm{~W}$ & & (U.S.A.) & & $50-60$ & 44 & $116.000^{3}$ & 29.000 & & & & -06 & Strehlow et al 1969 \\
\hline $190 \mathrm{~W}$ & Dec1duous, M & Bt. Louls, & , Area C & & 24 & $5.100^{3}$ & 0.710 & & & & -06 & Lockeretz $1975^{2}$ \\
\hline $191 \mathrm{~W}$ & Deciduous, $\mathrm{B}$ & St. Louls, & Area C & & 38 & $8.900^{3}$ & 0.790 & & & & -06 & Lockeretz $1975^{2}$ \\
\hline $192 \mathrm{~W}$ & Dec1duous, I & St. Louls, & Area C & & 48 & $10.100^{3}$ & 0.550 & & & & -06 & Lockeretz $1975^{2}$ \\
\hline $193 \mathrm{w}$ & Deciduous, iM & St. Louls, & Area I & & 15 & $6.200^{3}$ & 0.500 & & & & -06 & Lockeretz $1975^{2}$ \\
\hline $194 \mathrm{~W}$ & Deciduous, I & St. Louis, & Area I & & 27 & $10.800^{3}$ & 0.710 & & & & -06 & Lockerețz $1975^{2}$ \\
\hline $195 \mathrm{~W}$ & Deciduous, $M$ & St, Louls, & Area L & & 38 & $10.800_{3}^{3}$ & 0.840 & & & & -06 & Lockeretz $1975^{2}$ \\
\hline $196 \mathrm{~W}$ & Deciduous, B & St. Louls, & Area L & & 23 & $16.800^{3}$ & 1.540 & & & & -06 & Lockeretz $1975^{2}$ \\
\hline $197 \mathrm{~W}$ & Deciduous, I & st. Lours, & Area L & & 54 & $17.800^{3}$ & 1.260 & & & & -06 & Lockeretz $1975^{2}$ \\
\hline $198 \mathrm{~W}$ & Decíduous. M & St. Louis, & Area P & & 7 & $9.800^{3}$ & 2.000 & & & & -06 & Lockeretz $1975^{2}$. \\
\hline $199 \mathrm{~W}$, & Deciduous, I & st. Louls, & Area P & & 22 & $19.900^{3}$ & 1.530 & & & & -06 & Lockeretz $1975^{2}$ \\
\hline $200 \mathrm{~W}:$ & Deciduous, & St. Louis, & Area S & & 9 & $7.900^{3}$ & 1.050 & & & & -06 & Lockere'z $1975^{2}$ \\
\hline $201 \mathrm{~W}$, & Deciduous, I & St. Louis, & A-ea S & & 19 & $11.000^{3}$ & 0.810 & & & & -06 & Lockeretz $1975^{2}$ \\
\hline $202 \mathrm{~W}$ & & (U.S.S.R.) & & & & & & & & & & $\begin{array}{l}\text { Dmitriev } 1967 \text { 1 } \\
\text { Nezhivenko et al } 19671\end{array}$ \\
\hline $203 \mathrm{~W}$ & & $\begin{array}{l}\text { (U.S.S.R.) } \\
\text { (U.S.S.R.) }\end{array}$ & & & & 15.000 & & & 3.930 & 7.000 & $\begin{array}{l}-06 \\
-06\end{array}$ & $\begin{array}{l}\text { Nezhivenko et al } 1967^{\mathrm{I}} \\
\text { Storozheve } 1963^{1}\end{array}$ \\
\hline
\end{tabular}


TISSUE TYPE

$205 \mathrm{~W}$

Pd

$\begin{array}{ll}1 & \mathrm{E} \\ 2 & \mathrm{~W}\end{array}$

3 W, Restored

Px

$\begin{array}{lll}1 & \mathrm{E}, \mathrm{B} 1 \\ 2 & \mathrm{E}, \mathrm{B} 1\end{array}$

$\begin{array}{ll}2 & E, B 1 \\ 3 & E,\end{array}$

Pt

$1 \mathrm{D}$
$2 \mathrm{D}, \mathrm{Bl}$

$3 \mathrm{E}$
$4 \mathrm{E}, \mathrm{B1}$
$5 \mathrm{E}$

1 Ra

ํㅗ

\section{ra.}

$12 \mathrm{~W}$
$2 \mathrm{~W}$
$3 \mathrm{~W}$
$4 \mathrm{~W}$
$5 \mathrm{~W}$
$6 \mathrm{~W}$
$7 \mathrm{~W}$
$8 \mathrm{~W}$
$9 \mathrm{~W}$
$10 \mathrm{~W}$

$\mathbf{R b}$

1 D

$2 \mathrm{D}$, Une

$4 \mathrm{E}$

$5 \mathrm{E}, \mathrm{Bl}$

$6 \mathrm{E}$, Unerupted, $\mathrm{B}$

$7 \mathrm{E}$, Bl

$\begin{array}{lll}8 \mathrm{E}, & \mathrm{B} 1 \\ 9 \mathrm{E}, & \mathrm{B} 1\end{array}$

Ru

$\begin{array}{ll}1 & \mathrm{D} \\ 2 & \mathrm{E}\end{array}$

$s$

1 Calculus
2 Calculus
PROVENANCE

(U.S.S.R.)

(U.S.S.R.)

$>39$
$5-10$

$A \div E$

SAMPLES

15.000

15.000
3.500

(Freat Britain)

(Finlend)
(U.S.A.)

(U.S.A.)

(U.S.A.)

(Great Brt tain)

(Great Britain
Stockholm

(Great Britain)

(Sweden)

(U.

(U.S.A.)

(U.S.A.)

Region I

Region II

Japan

Misasa, Japan

Okayama, Japa

(J.S.A.)

(Great Britain)

(Sweden)
Stockholm

(Great Britain)

New Zealand

(Sweden)

(Sweden)

(U.S.A.)

(U.S.A.)

(Great Britain)

(Freat Britain)

(Japan)
(Japan)

$<20$
$<20$
$<20$
$<20$
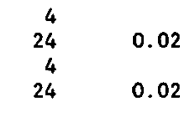

14-16

15-16

15

0.000

0.000

1.785
0.210

0.088

0.012

$14-25$
$14-16$
11
$14-25$
$1 \leq-16$
$<20$
$<20$

$\begin{array}{rr}10 & 69.000 \\ 15 & 5.600 \\ & \\ 36 & 0.440 \\ 10 & 73.000 \\ 15 & 4.900 \\ 28 & 0.390 \\ 56 & 0.410\end{array}$

45
45
ERROR

MEDIAN

MNN

0.000

0.030
0.030

$\begin{array}{rll}1 & 1.061 & \\ 10 & 1.061 & 0.117 \\ 1 & 1.785 & \end{array}$

0.003

52.000

0.030

0.030
56.000

2.200
0.030

0.030
0.020

0.320
0.360

0.320
0.360

000

0.170
0.140

MAX EXP

-06 Storozheva $1963^{1}$
-06 Storozheva $1963^{1}$
Zakson $1968^{1}$

$\begin{array}{rrrr}1.000 & -06 & \text { Hardwick et a1 } & 1967 \\ 10.000 & -06 & \text { Calontus et } 81 \quad 1965^{2}\end{array}$

$\begin{array}{llll}0.010 & -06 & \text { Losee et a1 } & 1974 \mathrm{~b} \\ 0.070 & -06 & \text { Losee et a1 } 1974 \mathrm{~b} \\ 0.010 & -06 & \text { Losee et a1 } 1974 \mathrm{c}\end{array}$

$0.070-06$ Losee et a1 $1974 \mathrm{C}$

1.000 $\begin{array}{rrl}10.000 & -06 & \text { Hardoick et al } 1967 \\ 0.06 & -06 & \text { Soremark et al 1964b } \\ 1.000 & -06 & \text { Harduick et al 1967 } \\ & -06 & \text { Sorremark et al } 19648\end{array}$

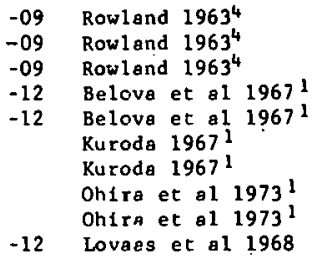

-12 Lhira et al 1973

1.000

10.1000

-06 Hardwick et al 1967

-06 Söremerk et al 1964b

10. $000-06$ Herdwick et al 1967

-06 Curzon et al 1975²

-06 Lundberg et al 1965a

-06 Soremark et al 1964a

$\begin{array}{lll}0.870 & -06 & \text { Losee et a1 } 1974 \mathrm{~b} \\ 0.870 & -06 & \text { Losee et }\end{array}$

$\begin{array}{llll}1.000 & -06 & \text { Hardwick et al } & 1967 \\ 1.000 & -06 & \text { Hardwick et al } & 1967\end{array}$

$\begin{array}{llll}590.000 & 1090.000 & -06 & \text { Inami } 1969 a^{1} \\ 910.000 & 2510.000 & -06 & \text { Inami } 1969 \mathrm{~b}\end{array}$ 


$\begin{aligned} 3 \mathrm{D} . & \text { (Great Brita1n) } \\ 4 \mathrm{D} & \text { (Japan) } \\ 5 \mathrm{D} & \text { (Sweden) } \\ 6 \mathrm{D}, \text { C1rcumpulpa1 } & \text { (Sweden) } \\ 7 \mathrm{D}, \text { Coronal } & \text { (Japan) } \\ 8 \mathrm{D}, \text { Root } & \text { (Japan) } \\ 9 \mathrm{D}, \text { Transparent } & \text { (Japan) } \\ 10 \mathrm{E} & \text { (Sreat Britain) } \\ 11 \mathrm{E} & \text { (Japan) } \\ 12 \mathrm{E}, \mathrm{Bl} & \text { New Zealand } \\ 13 \mathrm{E}, \mathrm{Bl} & \text { (U.S.A.) } \\ 14 \mathrm{E}, \mathrm{Bl} & \text { (U.S.A.) } \\ 15 \mathrm{E}, \text { Inner } & \text { (Sweden) } \\ 16 \mathrm{E}, \text { Outer } & \text { (Sweden) }\end{aligned}$

(Great Britain) (South Africa)
(South Africa) Denma rk

Denimark

Denmark

Denmark

Denmark

(Freat Britain)

(Freat Britain)

Egypt
New Zealand
(South Africa)

(South Africa)

(U.S.A.)

(U.S.A.)

Egypt

rilasgow

ilasgow

Glasgow

lasgow

(South Africa

(South Africa
(U.S.S.R.)

(Great Britain)

\section{$1 \mathrm{E}$}

Se

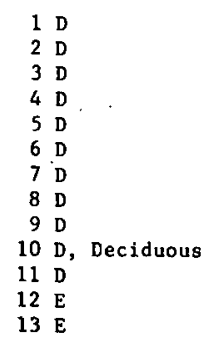

$27 \mathrm{~W}$

E

(Great Britain) ('reat Britain) Athens, Greece

Airginia

Virginia

Virginia

Virginia

(U.S.A.)

Portland, OR

(Great Britain)

Edinburgh

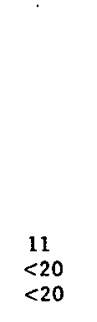

0.120

35
35

213.700

281.000

0.420

14.780

20.000
15.000

270.000
280.000

0.690
0.690

1100-1400 AD $1850 \mathrm{AD}$

$400 \mathrm{BC}-200 \mathrm{AD}$ 800-1100 AD

\section{$15-50$
11}

$<20$

$<20$
$15-50$

$14-61$
$14-61$

$14-33$

$14-61$
$14-61$

$24 \mathrm{E}$, Duter, Middle

$20-40$
$800-1100 \mathrm{BC}$
$10-12$
$13-16$
$17-24$
$>24$

$14-30$

$\begin{array}{rrr}100.000 & 1000.000 & -06 \\ 680.000 & 990.000 & -06 \\ & & -02 \\ & & -02 \\ 580.000 & 760.000 & -06 \\ 650.000 & 820.000 & -06 \\ 720.000 & 1170.000 & -06 \\ 10.000 & 100.000 & -06 \\ 26.000 & 70.000 & -06 \\ 130.000 & 530.000 & -06 \\ 110.000 & 560.000 & -06 \\ & 0.090 & -02 \\ & & -02\end{array}$

Hardwick et al 1967 Hachino $1969 a^{1}$ Ahlberg et al 1976 Hachino 1969 al.

Hachino 1969al

Hachino $1969 \mathrm{~b}^{\mathrm{l}}$

Hardwick et al 1967

Hachino 1969a
Curzon et al $1975^{2}$

Curzon et al $1975^{\circ}$
Losee et al 19746

Losee et al $1974 b$
Losee et al $1974 \mathrm{c}$

Ahlberg et al 1976

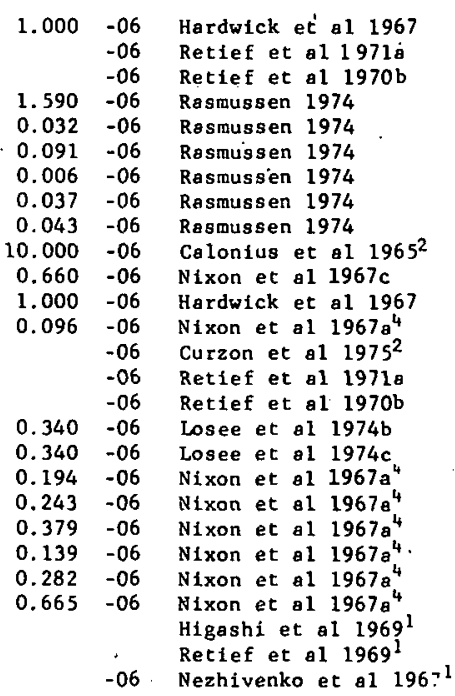

$1.000-06$. Hardwick et a1 1967

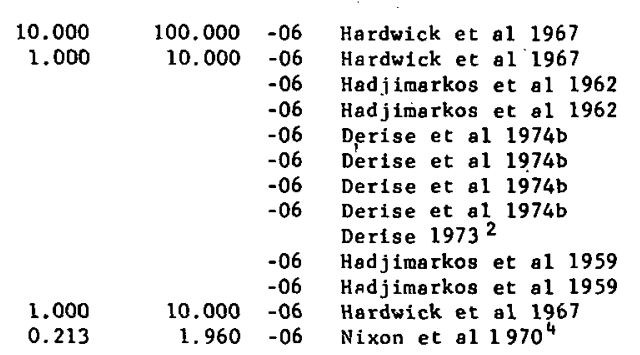




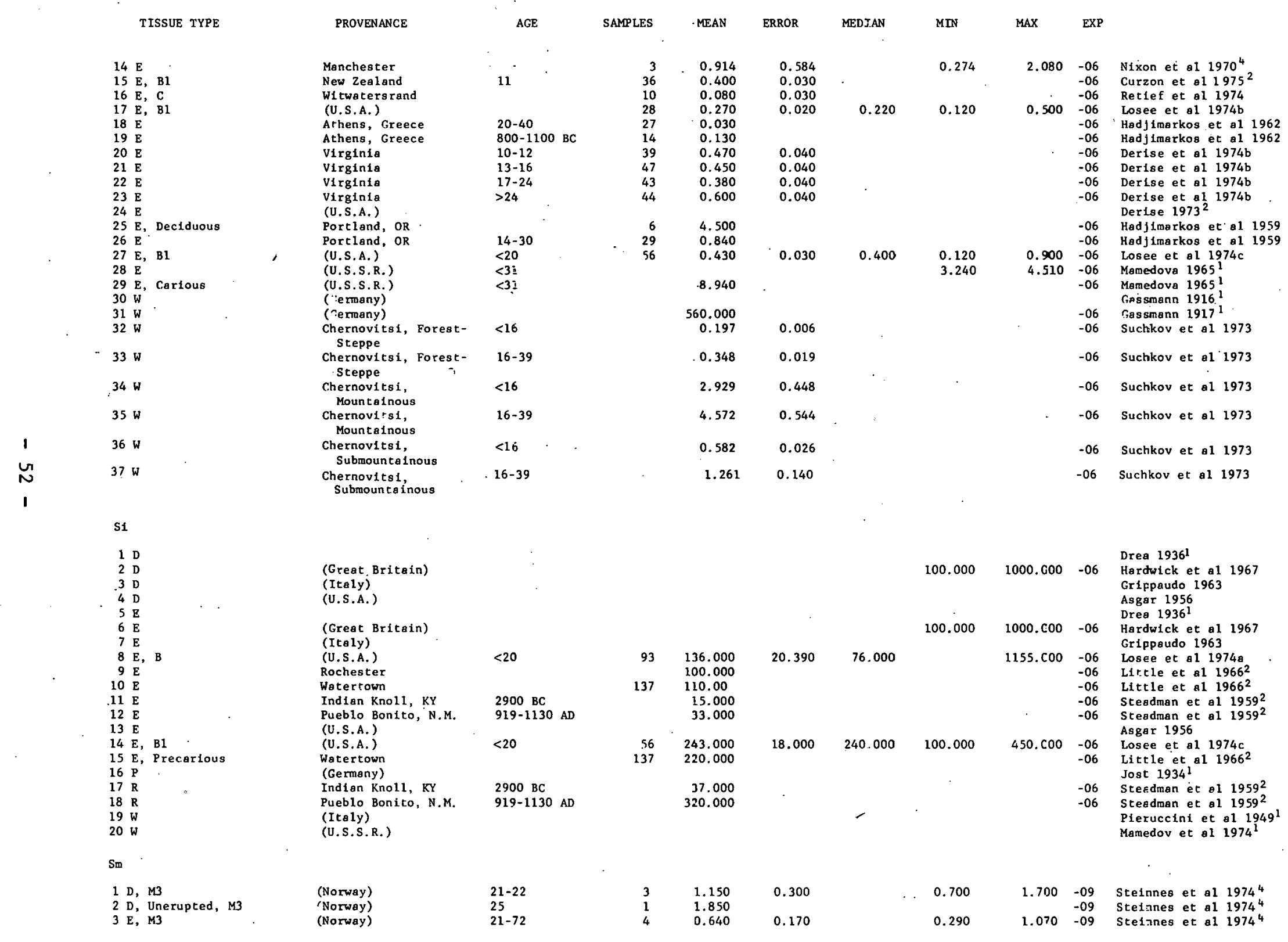


Sn

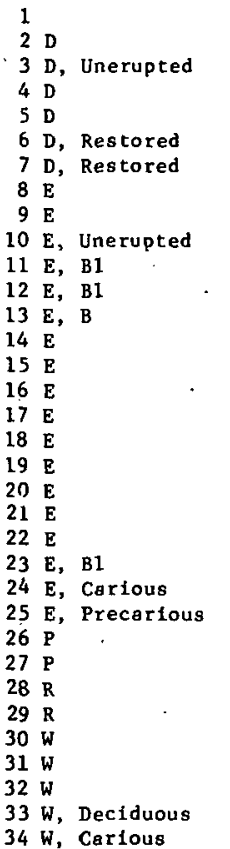

34 W, Carious

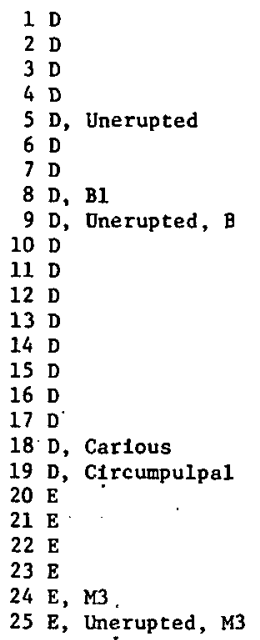

(U.S.A.)

(Great Britaln)

(Italy)

(U.S.A.)

(U.S.A.)

(Netherlands)

(Finland)

(Italy)

New Zealend

(U.S.A.)

(U.S.A.)

Watertown

(U.S.A.)

Indlan Knoll, $\mathrm{KY}$

Pueblo Bonito, N.M.

(U.S:A.)

(U.S.A.)

(U.S.A.)

Rochester

(Germany)

(Japan)

.

Pueblo BonIto, N.M.

(Finland)

(South Africa)

(U.S.S.R.)

(U.S.S.R.)

(Finland)

(Great Britain)

(Italy)

(South Africa)

(South Africa)

(Sweden)

(Sweden)

(Sweden)

(Sweden)

(Sweden)

Virginia

Virginta:

Virginia

(U.S.A.)

(Italy)

(Sweden)

(Finland)

(Great Britain)

(Isreal)

$>24$
93.000

11
$<20$
$<20$

2900 BC
$919-1130$ AD

$<20$

$2900 \mathrm{BC}$

$\begin{array}{rrrrr} & & & & 1.000 \\ & & & & 1.000 \\ 36 & 0.550 & 0.090 & & \\ 28 & 0.210 & 0.040 & 0.140 & 0.030 \\ 93 & 2.040 . & 1.060 & & \\ 137 & 1.000 & & & \\ & 2.500 & & & 0.000 \\ & 120.000 \\ & 3.300 & & & \\ & & & & \\ & & & & \\ 56 . & 0.530 & 0.140 & 0.230 & 0.030 \\ 137 & 24.000 & & & \\ & 12.300 & & & \\ & & & . & .\end{array}$

30

.0 .500
0.700

1.700

$15-16$
$14-25$

$94.330 \quad 11.470$

$94.330 \quad 4.720$

$\begin{array}{ll}69.800 & 18.000 \\ 64.000 & 18.000\end{array}$

$\begin{array}{rr}1 & 200.000\end{array}$

$10-12$
$13-16$
$17-24$

$\begin{array}{ll}39 & 179.000 \\ 47 & 218.900\end{array}$

$\begin{array}{lll}43 & 183.400 & 15.700 \\ 44 & 139.100 & 15.600\end{array}$

$1 \quad 190.000$

$21-29$
$21-29$

$\begin{array}{ll} & 119.400 \\ 15 \quad 138.800\end{array}$

$\begin{array}{ll}15 & 138.800 \\ 15 & 145.800\end{array}$

9,800
10.600
0.000

$\begin{array}{llll}1.000 & 10.000 & -06 & \begin{array}{l}\text { Glass 1967 } \\ \text { Hardwick et a1 1967 }\end{array}\end{array}$

-06 Gearlmain et al 1970 Pearlm

Asgar

Bex et al $1969^{1}$

$10.000-06$ Calonius et al $1965^{2}$

Hardwick et a1 1967

Trippaudo 1963

\begin{tabular}{ccc}
0.920 & -06 & Curzon et al $1975^{2}$ \\
\hline & -06 & Losee et al 1974b
\end{tabular}

$93.000-06$ Losee et a1 1974a

-06 Litrle et al $1966^{2}$

$7.000-06$ Brudevold et $a 11956 b^{2}$

-06 Pearlman. et al 1970

$\begin{array}{llll}0.500 & -06 & \text { Steadman et al } & 1959^{2} \\ \text { Steadman et al } & 1959^{2}\end{array}$

$3.000-06 \quad$ Asgar 1956

$7.100-06$ Wei $1974^{1}$

Losee et a1 1974

-06 . Johansen 1965

Little et a1 $1966^{2}$

Higashi et al $1969^{1}$

stogdmet al $1969^{2}$

$\begin{array}{rrr}0.500 & -06 & \text { Steadman et a1 } 1959^{2} \\ 10.000 & -06 & \text { Calong }\end{array}$

Calonius et a1 $1965^{\circ}$

Van Wyk et al 1968

Storozheva $1963^{1}$

Storozheva $1963^{1}$

-06 Storozheva $1963^{1}$

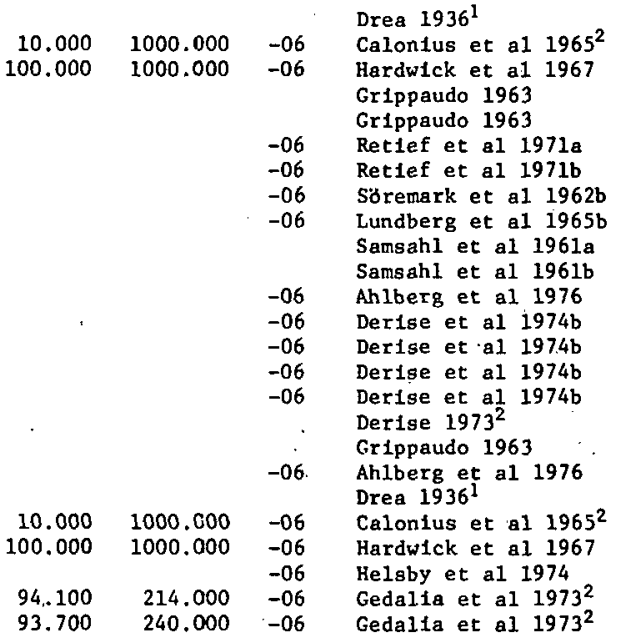




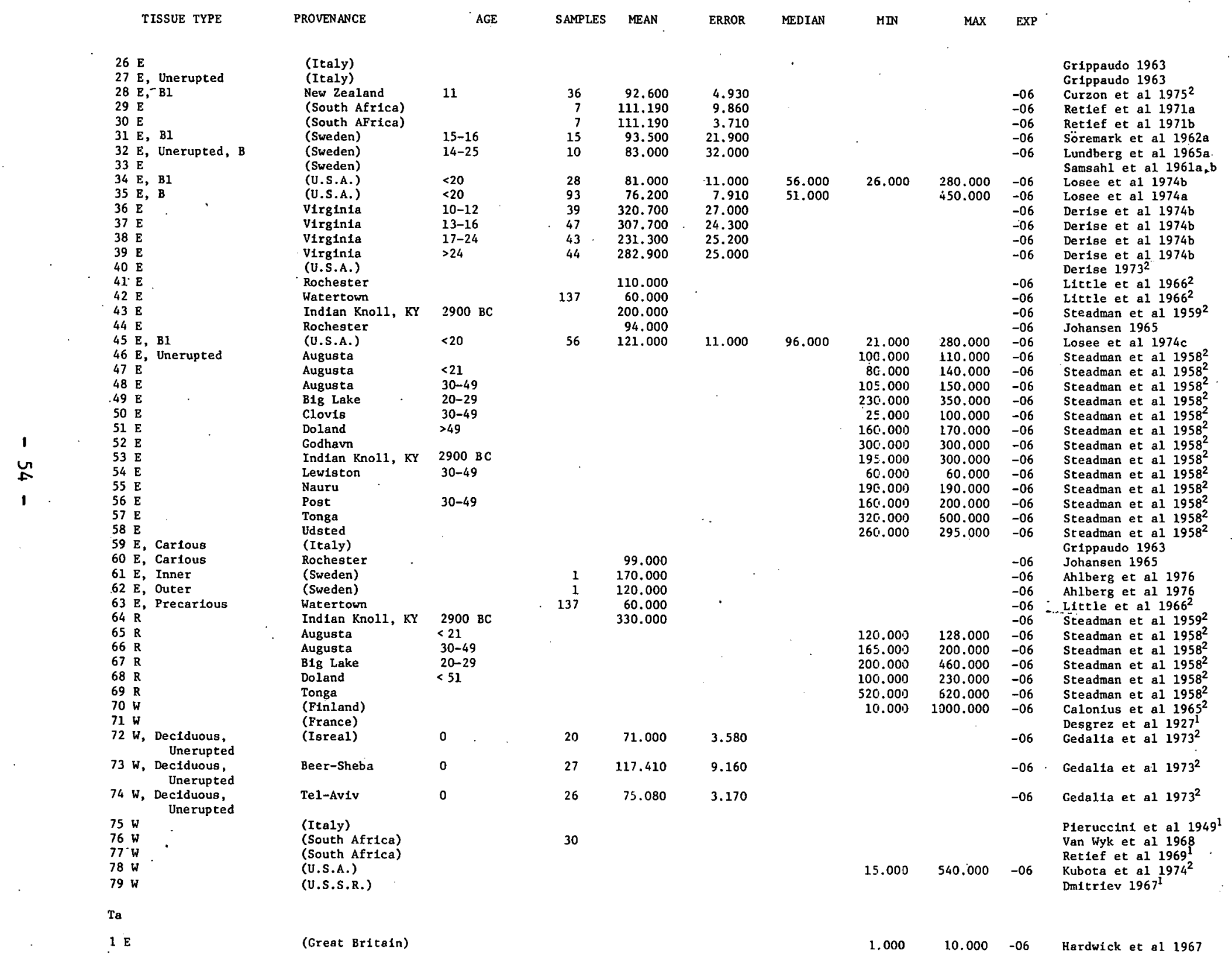


Tt

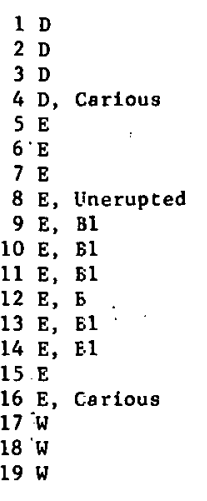
$2 \mathrm{~W}$ i $w$, Unerupted, $M$

(Great Britain)

(Italy)

(Great Britain)

(Iraly)
(Italy)

New Zealand

(U.S.A.)

(U.S.A.)

(U.S.A.)

(U.S.A.)

(U.S.S.R.)

(Italy)

(U.S.S.R.)

(U.S.S.R.)

(U.S.S.R)

(Great Britain)

(Japan)

Region II

$\begin{array}{ll}1 & D \\ 2 & D \\ 3 & D\end{array}$

4 D : Unerupted

4 D, Unerupt

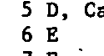

$8 \mathrm{E}$

$9 \mathrm{E}$, Unerupted

$10 \mathrm{E}$, Uner

$12 \mathrm{E}, \mathrm{B1}$

$13 \mathrm{E} ; \mathrm{B}$

$15 \mathrm{E}, \mathrm{B} 1$

$16 \mathrm{E}$, Carious

$17 \mathrm{~W}, \mathrm{M}$

$18 \mathrm{w}$,

$20 \mathrm{~W}$, Cartous

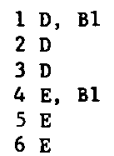

(Great Britain)

(Italy)

(Italy)

(Great Britain)

(Italy)

(Italy)

New Zealand
(U.S.A.)

(U.S.A.)

(U.S.A.)

(U.S.A.)

(U.S.S.R.)

(U.S.S.R.)

(U.S.S.R.)

(Sweden)

(Sweden)

(Sweden)

(Sweden)

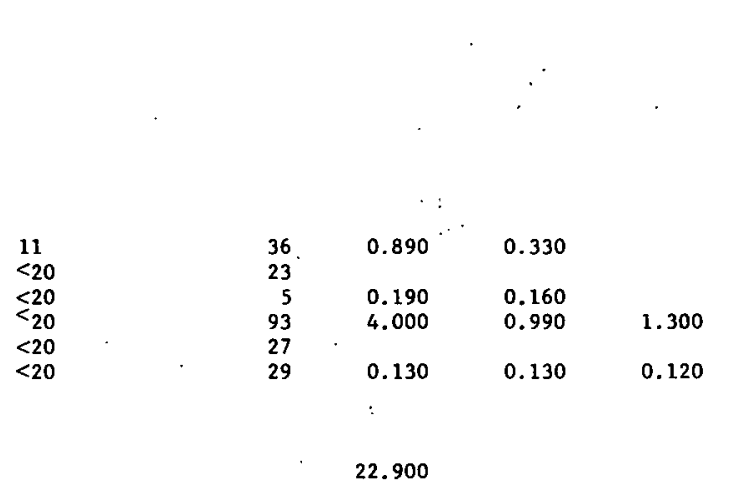

10.000

100.000

Drea $1936^{1}$

Hardwick et a1 1967

Srippaudo 1963 Drea $1936^{1}$

$\begin{array}{llll}10.000 & 100.000 & -06 & \text { Hardulck et al } 1967\end{array}$ Grippaudo 1963

irippaudo 1963

Curzon et al 197

$\begin{array}{lll}0.100 & -06 & \text { Losee et al 1974b } \\ 4.400 & -06 & \text { Losee et al 1974b }\end{array}$

$66.000-06$ Losee et a1 1974a

$4.800-06$ Losee et $811974 \mathrm{c}$

Dmitriev 19691

Grippaudo 1963

Mamedov $1974^{1}$

-06 Nezhivenko et al 1967

Zakson $1968^{1}$

$30-40$

$\begin{array}{rrr}15 & 7.000 & 1.000 \\ 3 & 34.330 & 5.780 \\ & 0.076 & \end{array}$

24.000

$44.000 \quad-09$

Hamil ton 1971

Tkeda et al $1967^{4}$

Belova et al 19671

1.000

$10.000-$

Drea $1936^{1}$

ick et al 1967?

Grtppaudo 1963

Grippaudo 1963

Drea 1936

23

$\begin{array}{lll}0.010 & -06 & \text { Nixon et a1 } 1967 \mathrm{c} \\ 1.000 & -06 & \text { Hardwick et ai } 1967\end{array}$

Grippaudo 1963

Grippaudo 1963

Curzon et al $1975^{2}$

Losee al $1974 \mathrm{~b}$

Curzon er a1 1974

Losee et al $1974 \mathrm{C}^{\circ}$

Grippaudo 1963.

Belekhova $1966^{1}$

Belekhova 1966

Mamedov et al $1974^{1}$

Belekhova et a1 $1966^{\circ}$

$-06$

Soremark et al 1962b

Semsehl et al 1961a

Samsahl et al 1961b

Soremark et al 1962a

Samsahl et. al 1961s 
TISSUE TYPE

Y

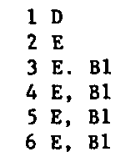

Zn

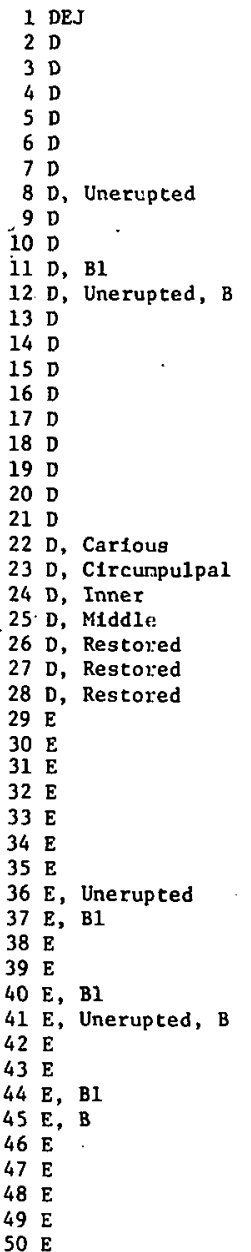

$49 \mathrm{E}$
SO E
PROVENANCE

AgE

(Great Britain)

(Great Britain)

(U.S.A.)

(U.S.A.)

(U.S.A.)

(U.S.A.)

(Finland)

(Great Britaln)

(Great Britain)

(Italy)

(South Africa)

(South Africa)

(Sweden)

(Sweden)

(Sweden)

(Sweden)

(Sweden)
V1rginta

Virginta
virginia

Virginia

virginia

(U.S.A.)

(Italy)

(Sweden)

(U.S.A.)

(U.S.A.)

(Netherlands)

(Sweden)

(Finland)

(Great Britain)

Cambridge

(Great Britain)

(Italy)

New Zealand

(South Africa)

(South Africa)

(Sweden)

(Sweden)

(Sweden)

(U.S.A.)

(U.S.A.)
Virginia

Virginta

Virginia

Virginte

$<20$

$<20$
$<20$

11

$15-16$
$14-25$

$<20$
$<20$

$<20$
$10-\mathrm{i} 2$

$13-16$
$17-24$
$>24$
0.007

0.000

0.007

.

$\begin{array}{lrrr} & & & \\ & & & \\ & & & \\ & 70 & 201.000 & \\ & & 169.000 & \\ & & & \\ & 9 & 172.810 & 11.140 \\ 15-16 & 9 & 172.810 & 3.710 \\ 14-25 & 15 & 199.000 & 78.100 \\ & 10 & 160.000 & 30.000 \\ & & & \\ 10-12 & 1 & 150.000 & \\ 13-16 & 39 & 148.900 & 8.900 \\ 17-24 & 47 & 185.700 & 8.000 \\ >24 & 43 & 194.700 & 8.200 \\ & 44 & 170.400 & 8.300 \\ & & & \end{array}$

$1 \quad 510.000$

$130 \quad 365.000$

$99 \quad 197.000$ 116.000
ERROPR

MEDIAN

MIN

EXP

\begin{tabular}{|c|c|c|c|}
\hline 150.000 & 300.050 & -06 & $\begin{array}{l}\text { Brucievold et a1 } 1963^{2} \\
\text { Drea 1936 }\end{array}$ \\
\hline 1.000 & 10.030 & -06 & Calonius et al $1965^{2}$ \\
\hline \multirow[t]{16}{*}{100.000} & 1000.030 & $\begin{array}{l}-06 \\
-06\end{array}$ & $\begin{array}{l}\text { Harciw1sk et a1 } 1967 \\
\text { Crutckshank } 1949^{2}\end{array}$ \\
\hline & & & Bab1cky et a1 $1966^{1}$ \\
\hline & & & Gr1ppaudo 1963 \\
\hline & & -06 & $\begin{array}{l}\text { Grippaudo } 1963 \\
\text { Retiof }\end{array}$ \\
\hline & & $\begin{array}{l}-06 \\
-06\end{array}$ & $\begin{array}{l}\text { Retief et al } 1971 \text { a } \\
\text { Retlef et al } 1970\end{array}$ \\
\hline & & -06 & Söremark et al 1962b \\
\hline & & & $\begin{array}{l}\text { Luncberg et a1 1965b } \\
\text { Samsah1 et al 1961a }\end{array}$ \\
\hline & & & Samsahl et al 1961b \\
\hline & & & Ahlberg et al 1976 \\
\hline & & -06 & Derise et al 1974b \\
\hline & & -06 & Derise et al 1974b \\
\hline & & -06 & Derise et al $1974 \mathrm{~b}$ \\
\hline & & -06 & Derise et a1 1974b \\
\hline & & & DerIse $1973^{2}$ \\
\hline & & & Asgar 1956 \\
\hline & & -06 & Ahlterg 1976 \\
\hline 560.000 & 1400.000 & -06 & Brudevold et a1 $1963^{2}$ \\
\hline 200.000 & 300.0100 & -06 & Brudevold et al $1963^{2}$ \\
\hline & & & Kurcsak1 et a1 $1973^{1}$ \\
\hline & & & Bax et al $1969^{1}$ \\
\hline & & & $\begin{array}{l}\text { Söremark et al } 1968^{2} \\
\text { Drea } 1936^{1}\end{array}$ \\
\hline 10.000 & 1050.000 & & Calcntus et al $1965^{2}$ \\
\hline 58.000 & 932.000 & -06 & N1xen et al $1967 \mathrm{c}$ \\
\hline \multirow[t]{10}{*}{100.000} & 10100.000 & -06 & Hardwick et a1 1967 \\
\hline & & -06 & Cruickshank $1949^{2}$ \\
\hline & & & Bab1cky et al $1966^{1}$ \\
\hline & & & $\begin{array}{l}\text { Grippaudo } 1963 \\
\text { Grippaudo } 1963\end{array}$ \\
\hline & & -06 & Curzon et al $1975^{2}$ \\
\hline & & -06 & Rettef et al 1971a \\
\hline & & -06 & Retlef et al 1970 \\
\hline & & -06 & Söremark et al 1962a \\
\hline & & -06 & Lundberg et al 1965a \\
\hline & & & et al 196 \\
\hline \multirow[t]{6}{*}{91.000} & 400 & & Losee et al 1974b \\
\hline & 1197.000 & -06 & Losee et al 1974a \\
\hline & & -06 & Derise et al 1974b \\
\hline & & -06 & Derise et al 1974b \\
\hline & & -06 & Derise et al 1974b \\
\hline & & -06 & Derise et al 1974b \\
\hline
\end{tabular}

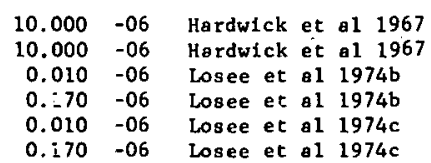
.

$\begin{array}{rrrr}36 & 151.400 & 14.170 & \\ 8 & 253.420 & 14.800 & \\ 8 & 253.420 & 5.230 & \\ 15 & 276.000 & 106.000 & \\ 10 & 215.000 & 41.000 & \\ & & & \\ 28 & 199.000 & 14.000 & 190.000 \\ 93 & 241.400 & 12.860 & 213.000 \\ 39 & 190.600 & 4.100 & \\ 47 & 192.700 & 3.700 & \\ 43 & 179.200 & 3.800 & \\ 44 & 180.300 & 3.800 & \end{array}$

Derise et al $1974 b$
Derise $1973^{2}$ 


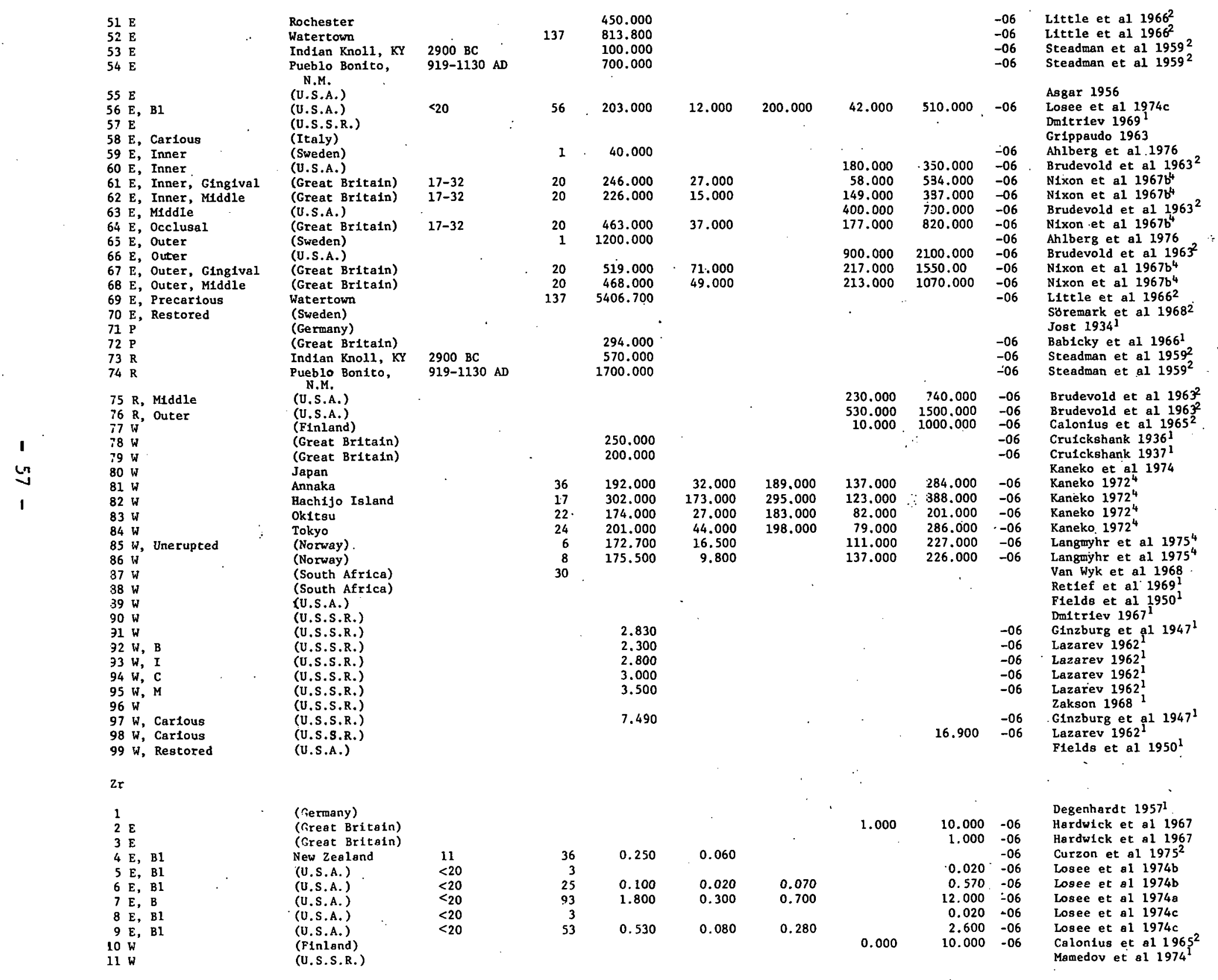


FOOTNOTES

1. Entry based on citation in Chemical Abstracts.

2. Reference features more data than appears in this tabulation.

3. Value based on geometric mean.

4. Reference lists data on individual samples. 


\section{$\underline{\underline{2.2}} \underline{\underline{\text { Statistical analysis }}}$}

Analysis of variance and covartance are the principal techniques by which we have analyzed compositional data in this survey. Because of the (largely) unknown natures of the populations from which specimens have been obtained, our focus has been on "fixed-effects" models where the inferences made do not extend beyond the data actually collected and analyzed.

The models we employ are of two types, univariate and multivariate. The two univariate models we use and the associated null hypotheses are:

I. One-way model

$$
\begin{aligned}
& x_{\underline{i j}}=\theta_{\underline{j}}+\varepsilon_{\underline{i j}} ; \underline{j}=1, \underline{k} ; \underline{i}=1, N_{\underline{j}} \\
& H_{0}: \theta_{1}=\theta_{2}=\ldots=\theta_{\underline{k}}
\end{aligned}
$$

II. One-way model with one covariate

$$
\begin{aligned}
& x_{\underline{i j}}=\theta_{\underline{j}}+\alpha z_{\underline{i j j}}+\varepsilon_{\underline{1} \underline{j}} ; \underline{1}=1, \underline{k} ; \underline{1}=1, N_{j} \\
& H_{0}: \theta_{1}=\theta_{2}=\ldots=\theta_{k} \\
& H_{0}: \alpha=0
\end{aligned}
$$


In both models, $\varepsilon_{\underline{i j}}$ is a random error term and $\theta_{\underline{j}}$ and $\alpha$ are fixed parameters. In Mode1 II, $z_{i j}$ is a covariate of known value. The assumptions underlying these models and the standard F-tests of the hypotheses may be found in Scheffé (1959).

Much of the data we have analyzed is multivariate; that is, for each experimental unit, such as a tooth, several measurements have been made. Among the types of multivariate data encountered in the dental literature, one derives from broad spectrum surveys where the concentrations, of two.or more elements are measured in each sample. A second type occurs when the concentration(s) of one (or more) element(s); are measured in each of two or more types of tissue from the same sample (tooth). Where more than one response is measured for each experimental unit, the errors in the several ' responses are frequently correlated. Multivariate statistical techniques allow for this correlation and, in addition, provide confidence intervals that allow contrasting of main effects (regional differences in composition, for example) simultaneously for each of the responses.

Two of the four multivariate models we employ are simple extensions of equations ( 8 ) and (10),

\section{I: One-way mode1} $\therefore$

$$
\ddot{x}_{\underline{1 j \underline{h}}}=\theta_{\underline{j \underline{h}}}+\varepsilon_{\underline{i j h}} ; \underline{j}=1, \underline{k} ; \underline{i}=1, N_{\underline{j}} ; \underline{h}=1, \underline{p}
$$

\section{II: One-way model with one covariate}

$$
x_{\underline{i j \underline{h}}}=\theta_{\underline{j \underline{h}}}+\alpha_{\underline{h}} z_{\underline{i j}}+\varepsilon_{\underline{1 j} \underline{\underline{h}}} ; \underline{j}=1, \underline{k} ; \underline{i}=1, N_{\underline{j}} ; \underline{h}=1, \underline{\underline{h}}
$$

where $\mathrm{p}$ is the number of responses indexed by $\underline{h}$. The third is a linear regression model of the form, 
III: Linear Regression

$$
\underline{x_{i h}}=\theta_{h}+\alpha_{h \underline{i}}+\varepsilon_{\underline{i h}} ; \underline{1}=1, N ; \underline{h}=1, \underline{p}
$$

and the fourth is a general linear model of the form,

IV. A general linear model

$$
x_{\underline{1} \underline{j} \underline{h}}=\theta_{\underline{j h}}+\alpha_{\underline{j h} \underline{\underline{1}} \underline{j}}+\varepsilon_{\underline{1 j \underline{h}}} ; \underline{j}=1, \mathrm{k} ; \underline{1}=1, \mathrm{~N}_{\underline{j}} ; \underline{\mathrm{h}}=1, \mathrm{P}
$$

Several hypotheses may be formulated and tested. For

I, these are:

$$
\begin{aligned}
& H_{0}: \theta_{1 \underline{h}}=\theta_{2 \underline{h}}=\ldots=\theta_{\underline{k} \underline{h}}, \underline{h}=1, \underline{p} \\
& H_{0}: \sum_{\underline{j}=1}^{k} \theta_{j 1}=\sum_{\underline{j}=1}^{\frac{k}{j 2}}=\ldots=\sum_{j=1}^{\underline{j} \underline{p}}
\end{aligned}
$$

for II,

$$
\begin{aligned}
& \mathrm{H}_{0}: \theta_{\underline{1 \underline{h}}}=\theta_{2 \underline{\mathrm{h}}}=\ldots=\theta_{\underline{\mathrm{kh}}}, \underline{\mathrm{h}}=1, \mathrm{p} \\
& \mathrm{H}_{\mathrm{O}}: \alpha_{\underline{\mathrm{h}}}=0 \quad, \underline{\mathrm{h}}=1, \mathrm{P} \\
& H_{0}: \sum_{\underline{j}=1}^{\frac{k}{\Sigma}} \theta_{\underline{j 1}}=\underbrace{\frac{k}{\Sigma}}_{\underline{j}=1} \theta_{\underline{j 2}}=\ldots=\underbrace{\frac{k}{\Sigma}}_{\underline{j=1}} \theta_{\underline{j p}}
\end{aligned}
$$

for III,

$$
\begin{aligned}
& \mathrm{H}_{0}: \alpha_{\underline{h}}=0 \\
& \mathrm{H}_{0}: \theta_{1}=\theta_{2}=\ldots=\theta_{\underline{\mathrm{p}}}
\end{aligned}
$$


and for IV,

$$
\begin{aligned}
& \underline{H}: 0 \theta_{1 \underline{h}}=\theta_{2 \underline{h}}=\ldots=\theta_{\underline{\mathrm{kh}}} \quad, \underline{\mathrm{h}}=1, \mathrm{p} \\
& \mathrm{H}_{\mathrm{o}}: \alpha_{\underline{j h}}=0 \quad \therefore \quad, \ldots, \underline{j}=1, \underline{\mathrm{k}} ; \underline{\mathrm{h}}=1, \mathrm{p} \\
& \mathrm{H}_{0}: \sum_{j=1}^{\underline{k}} \theta_{j 1}=\sum_{j=1}^{\underline{k}} \theta_{j 2}=\ldots=\sum_{\underline{j}=1}^{\underline{k}} \theta_{\underline{j} \underline{p}}
\end{aligned}
$$

The last hypothesis for each model may be termed the hypothesis of equal response effects; the first hypothes'es for models I, II, and IV may be termed the hypotheses of equal main effects; and the first hypothesis for III and the second hypotheses for models II and IV may be termed the hypotheses of no regression relationship. The separate hypotheses are tested with Roy's statistic (the 'greatest root' distribution) [Morrison (1976)] which reduces to an F-test for the data at hand, except in one instance. The assumptions underlying these models and hypothesis tests can also be found in Morrison (1976).

For both univariate and multivariate models, the assumption of most concern to us is the requirement that the expectation of the error, $E(\varepsilon)$, is zero. This is another way of requiring that the model is correct; that it provides a true account of the dependence of the measured data on the subscripting variables $\underline{\underline{i}}$ and $\underline{j}$ (and $\underline{h}$ ). This condition is rarely fulfilled 
exactly since any model chosen is almost certainly inadequate, and this applies particularly to biochemical data. In many cases, the adequacy of a model has to be judged in relation to the overall goals of the study. Is the study exploratory? or is it designed to be definitive? In many respects, the statistical analyses we provide in sections 2.3 through 2.7 are exploratory. The inferences made are tentative. Inferences derived from hypothesis tests based on "fixed-effects" models are, as observed earlier, restricted to the sample of data analyzed. From the standpoint of generality, "random-effects" or "mixed-effects" models [Scheffe (1959)] may be of more value in that they permit inferences regarding the population from which the sample of data was obtained. To be of utility, however, this population must have been defined and properly sampled. For all but well-structured data (i.e., "balanced" designs), the computational labor associated with these models can be formidable [Harville (1977)]. Their statistical properties are fundamentally different from those of "fixed-effects" models.

Having rejected a null hypothesis, and being satisfied that the model employed is adequate, one may be interested in ascertaining which combination of $\theta_{j}$ (or $\theta_{j h}$ ) and/or $\alpha$ (or $\alpha_{h}$ ) lead to this rejection. One might (depending on one's interests) accomplish this for the $\theta_{\underline{j}}$ by forming differences, $\hat{\theta}_{\underline{j}}-\hat{\theta}_{\underline{k}}$, between pairs of estimates and computing confidence intervals. Pairs for which the confidence interval does not include zero are then significantly different. Such pairwise differences are examples of what are called contrasts. As long as contrasts are formed a priori, Student's $\underline{t}$ can be used to compute confidence intervals. Where several contrasts are formed, confidence intervals evaluated simultaneously, and some assurance desired that the family of contrasts will have a given error rate ( $5 \%$, say), different techniques should be used 
[Miller (1966), see.pp.1-12 for an account of why and where procedures for simultaneous inference are appropriate.]. These generally lead to confidence intervals that are broader than those obtained with Student's $\underline{t}$. Finally, one should bear in mind that though the null hypothesis is rejected, all the confidence intervals for all pairwise contrasts may include zero! For the hypothesis tests we have employed, this rather disconcerting observation has its origin in the mathematics used to derive the tests [Morrison (1976)] and, if one's primary interest is in the pairwise. contrasts, one might well ascribe rejection of the null hypothesis to artefact. One should also be aware that if the decision to investigate differences between main effects using simultaneous confidence intervals is conditional on rejection of the null hypothesis in an analysis of variance, then the statistical properties of these intervals change. [This would seem to be largely a matter of concern to professional statisticians. Some recent discussion and reference to earlier literature may, be found in Scheffé (1977 a, b) and Olshen (1977).]

Compositional data are sometimes transformed (the only transformation employed in the literature surveyed by us has been the logarithmic transformation) in order to achieve a more nearly normal distribution. Much of the data on the inorganic composition of dental hard tissues is skewed towards lower concentrations, particularly for trace constituents, and appears approximately log-normally distributed. Strictly speaking, one is more interested in whether the errors in the data are log-normally distributed. In analytical chemistry, where concentrations are frequently known to have a constant percent error, this is approximately true, but the analytical error is not the same as the departure of the assumed model from the true concentration. This error includes the analytical error and an error component due to failure of the model to describe reality. Nevertheless, transformation prior to statistical analysis may be desirable though it is often based on subjective 
examination of a histogram of the complete sample of concentration data or on knowledge of the fact that the concentration data may range over several orders of magnitude.

The logarithmic transformation is but a special case of a much broader class of power transformations that have been investigated statistically by Box and Cox (1964) and.by Andrews et al (1971). These more general transformations, however, have not been employed in the dental literature: It would be interesting to know how, if at all, compositional data deviates from either the normal or log-normal distribution (as measured by estimates of the general power traneformation). The same model, however, employed with transformed data is fundamentally different, mathematically and statistically, than if employed with untransformed data.

Among the more troublesome problems one must often confront in the analysis of compositional data, are those associated with missing and censored data. Techniques for handling missing response data in the univariate analysis of variance are well developed [Rubin (1976)]; the same, however, is not true for multivariate models. Hartley and Hocking (1971) have derived methodologies applicable to estimation in the multivariate case which, however, are computationally complex. Very little is known about hypothesis testing for these models.

Missing data, by its nature, may take on any value allowable under the distributional assumptions adopted. Censored data, however, is data known to be less than (or greater than) some (known) value. Censored data is far more common in broad spectrum multielement studies where the probability is considerable that one or more elements will be at concentrations lower than instrumental detection limits in each sample, than it is in single element studies where analytical techniques may be chosen in a manner that minimizes the occurrence of such data. Whereas In many instances, missing data can be handled simply by deleting it from the statistical analysis, the same procedure is not appropriate for censored data: Censoring will not always be a random event. 
Any large-scale study of the inorganic composition of dental hard tissues will have to involve development and testing of procedures for the analysis of censored data. At an elementary level, this may involve use of existing techniques. Cohen (1959) [see also literature cited by Cohen], for example, provides means of estimating the mean and variance from a censored univariate normal population. Applied to Kaneko's data on Cd [Kaneko (1972)], assumed to be $\log$-normally distributed with a limit of detection of $0.01 \mathrm{ppm}$, this technique yields the results given in Table III. We are not aware of the existence of published results on estimation with censored multivariate data: Such procedures, if they exist or are to be developed, will probably be based on maximum likelihood estimation (we are currently working on maximum likelihood estimation of the principal components of censored multivariate data). The data (or some marginal transformation of it) will probably be assumed to have come from a censored multivariate normal population. The requisite equations, though not difficult to derive, may prove computationally cumbersome to solve. There are no simple solutions, however, and eventually the computational task must be confronted. The related problem of obtaining hypothesis tests and confidence intervals applicable to censored data is much more difficult and to our knowledge, little relevant statistical theory has been developed. (Hartley and Hocking (1971) give some results on confidence intervals for grouped -- a type of censored -- univariate data.) One might hope, however, that after the necessary quantities are estimated, standard hypothesis tests can be employed. Where the fraction of censored data is small, this is probably not unreasonable. Much remains to be done in this area, however. 
Table III. Maximum-Likelihood ${ }^{1}$ Estimates of ('Geometric') Mean Cadmium Levels in Teeth [Data from Kaneko (1972)]

\begin{tabular}{|c|c|c|c|c|c|c|c|c|c|c|}
\hline & $\mathrm{N}$ & $\underline{\mathrm{n}}$ & $\overline{\mathbf{x}}$ & $\mathbf{s}$ & $\hat{\mu}$ & $\hat{\sigma}$ & $10^{\bar{x}}$ & $(\mu g / g)$ & $10^{\hat{\mu}}$ & $(\mu g / g)$ \\
\hline Tokyo & 24 & 19 & -1.086 & -1.710 & -1.333 & -1.443 & & 0.08 & & 0.05 \\
\hline okjtsu & 21 & 12 & -1.140 & -1.785 & -1.723 & -1.260 & & 0.07 & & 0.02 \\
\hline Hachijo ${ }^{2}$ & 17 & 17 & -0.370 & -1.603 & -0.370 & -1.603 & & 0.43 & & 0.43 \\
\hline Annaka & 35 & 32 & -0.998 & -1.744 & -1.096 & -1.595 & & 0.10 & & 0.08 \\
\hline
\end{tabular}

N, total number of teeth analyzed.

n. number of teeth in which cadmium levels were above detection limits.

$\overline{\mathbf{x}}, \quad\left(\begin{array}{cc}\frac{n}{\sum_{i=1}} & \log _{10} Y_{i}\end{array}\right) / \underline{n}, \begin{gathered}\left(y_{i} \text { is the cadmium concentration in }\right. \\ \text { sample } i) .\end{gathered}$

s, $\left[\left(\sum_{i=1}^{\frac{n}{\Sigma}}\left(\log _{10} y_{i}-\bar{x}\right)^{2}\right) / n\right]^{\frac{1}{2}}$.

$\hat{\mu}, \hat{\sigma}$, censored data estimates of $\mu$ and $\sigma$ based on $N, n, \bar{x}$, and $s$, and an assumed limit of detection of $0.01 \mu \mathrm{g} / \mathrm{g}$.

\footnotetext{
1 Assuming the data derived from a log-normal distribution.

2 No censoring occurred in this case; hence, $\mu=\bar{x}$, and $\hat{\sigma}=s$.
} 


\section{$\underline{\underline{2.3}} \underline{\underline{\text { Geographic variation }}}$}

Evidence exists suggesting the importance of relationships between the trace element content of dental hard tissues and their provenance (the donor's residence). The purpose of this section is to review this evidence.

The trace element content of enamel samples derived from pairs of upper left and right first bicuspids from 18 New Zealanders has been compared with compositional data on enamel derived from 56 first bicuspids from residents of the United States [Curzon et al (1975)]. Of the 25 elements compared (Ag, Al, $\mathrm{B}, \mathrm{Ba}, \mathrm{Br}, \mathrm{Cd}, \mathrm{Cu}, \mathrm{F}, \mathrm{Fe}, \mathrm{Li}, \mathrm{Mn}, \mathrm{Mo}, \mathrm{Nb}, \mathrm{Ni}, \mathrm{Pb}, \mathrm{Rb}, \mathrm{S}, \mathrm{Sb}, \mathrm{Se}, \mathrm{Sn}, \mathrm{Sr}, \mathrm{Ti}, \mathrm{V}$, $\mathrm{Zn}$, and $\mathrm{Zr}$ ), the concentrations of 15 elements were judged by Curzon et al (1975) significantly different, regionally, on the basis of univariate $\underline{t}$-tests at a level significance of $5 \%$. These elements were $\mathrm{Ag}, \mathrm{B}, \mathrm{Br}, \mathrm{Cd}, \mathrm{Cu}, \mathrm{F}, \mathrm{Fe}, \mathrm{Mn}$, Mo, $\mathrm{Nb}$, $\mathrm{Pb}, \mathrm{S}, \mathrm{Sr}, \mathrm{Zn}$, and $\mathrm{Zr}$. Some differences were highly significant $(\alpha=0.001)$. Kaneko (1972) and Kaneko et al (1974) describe work on the $\mathrm{Ca}, \mathrm{Cd}, \mathrm{Cu}, \mathrm{Pb}$, and $\mathrm{Zn}$ contents of 99 whole teeth from four regions in Japan characterized as urban (Tokyo), rural (Okitsu), fishing village (Hachijo Island), and cadmium contaminated (Annaka) environments. They compared regional differences for each element and, on the basis of univariate t-tests, applied to log-transformed data, concluded that some of these differences were significant. For a significance level of $5 \%$, the Tokyo-Okitsu comparison was significant for $\mathrm{Ca}, \mathrm{Cu}$, and. $\mathrm{Pb}$; the Tokyo-Hachijo comparison was significant for $\mathrm{Cd}, \mathrm{Cu}$, and $\mathrm{Zn}$; the Tokyo-Annaka comparison was significant for $\mathrm{Ca}, \mathrm{Cu}$, and $\mathrm{Pb}$; the Okitsu-Hachijo comparison was significant for $\mathrm{Ca}, \mathrm{Cd}, \mathrm{Cu}, \mathrm{Pb}$, and $\mathrm{Zn}$; the Okitsu-Annaka comparison was significant for $\mathrm{Zn}$; and the Hachijo-Annaka comparison was significant for $\mathrm{Ca}, \mathrm{Cd}, \mathrm{Cu}$, and $\mathrm{Zn}$. For Ra and U, Belova et al (1967) reports levels for human teeth for two Soviet regions; tooth content parallels closely, levels measured for vegetable products and water for these regions, although no measure of significance is provided. Similar results have been reported by Kuroda (1967) for the Po and 
Ra contents of teeth from two Japanese regions whose background radioactivities differed by a factor of 1.5. The difference between teeth of these two regions wịth respect to Po content was significant $(\alpha=0.01)$. In studies of human teeth from Tottori prefecture and Okayama in Japan, Ohira et al (1973) report significant regional differences in the ${ }^{210} \mathrm{~Pb}$ and $\mathrm{Ra}$ contents of teeth and some foods.

of studies dealing with regional differences in the elemental composition of teeth, those devoted to single elements are most common. Two elements have been of particular interest: Fluorine and, more recently, lead. Regional differences have been reported in the $\mathrm{F}$ content of enamel and dentine [McClure and Likins (1951); Jackson and Weidmann (1959); and Shaw et al (1959)] and of enamel, dentine, and root tissue [Yoon et al (1960)]. Jackson and Weidmann (1959), for example, studied on a regional basis (Leeds, South Shields, and West Hartlepool), the relationship between age and the fluorine content of ename1 and dentine and, in every age category, found that those teeth from regions with higher water fluorine content had greater $F$ levels in dental tissues. Although the differences in dental fluorine levels between regions were not tested for significance, sufficient data are given for such tests to be carried out. (Visual inspection of the data leaves little doubt about the outcome of such testing, however.) In view of the evident effect of age on the data and on the basis of graphical presentation given by Jackson and Weidmann (1959), we have chosen to fit the following equation to their data, making no allowance, however, for unequal variances between age categories:

$$
F(m g / 100 g)=\theta_{1}+\theta_{2} \exp \left(-\theta_{3} t\right)+\varepsilon
$$

While the number of degrees of freedom for hypothesis tests on the equality of $\theta_{1}$, say, among the three regions are too small for reliable testing, two points of interest emerge from this analysis: (i) the ratios of $\theta_{1}$ for West Hartlepool, 
South Shields, and Leeds are 2.1:1:0.6 for enamel and 2.2:1:0.6 for dentine, while the ratios of the fluorine levels in the three water supplies are $2: 1:<0.5$. The parallel is remarkable. (ii) The ratios of $1 / \theta_{3}$ for West Hartlepool, South Shields, and Leeds are 2.2:1:0:3 for enamel and 2.4:1:0.9 for dentine. The parallel between these results and the fluorine levels in the three water supplies, though less remarkable, is nevertheless noteworthy. $\theta_{1}$ is a measure of the 'saturation: level of fluorine in dentine and enamel, while $\theta_{3}$ is a measure of how fast saturation is approached as a function of age. That both appear to be functions of ambient drinking water fluorine levels is interesting; beyond that, however, little biological significance can presently be ascribed to this model. Shaw et al (1959) report variation of fluorine levels in enamel and dentine on a global basis in a survey involving residents of the United States, India, Iran, Pakistan, and the Canadian Arctic. They have stratified their data with respect to country and city of residence and, in the case of teeth from the Canadian Arctic; with respect.to race and living conditions. Differences between cities within India and between population groups within Canada were analyzed statistically for significance, and differences between countries were discussed. The data reported are sufficient to permit removal of stratification with respect to city of residence (India) and race and living conditions (Canadian Arctic); this we have done in order to provide a set of statistics for testing differences on a national level (a reflection of gross geographical variation). Appropriately constructed 95\% simultaneous confidence intervals [Miller (1966)] lead to the rejection of the null hypothesis of equal population (national) means for the comparisons: For dentine--U.S.-Canada; U.S.-India; U.S.-Iran; U.S.-Pakistan, and Canada-Iran: For enamel--U.S.-Canada; U.S.-India; U.S.-Iran; U.S.-Pakistan, and Canada-India. Most of the contrasts 
involving the U.S. are significant at $0.5 \%$ and result, as observed by Shaw et al (1959), from the low fluorine concentrations in teeth obtained from Boston (the only source of U.S. teeth). McClure and Likins (1951) describe analyses of the fluorine content of enamel and dentine from residents of eight regions with known fluorine levels in local water supplies that demonstrate a relationship between water and dental. F concentration. No variation in $F$ with age was found, however, though this may have resulted from the broad age categories used to categorize the data $(8-25,25-45,>45)$. Yoon et al (1960) report on the distribution of fluorine in tceth from six communities with varying levels of fluorine in local water supplies and although no measure of variability can be attached to the data and hence no hypothesis testing carried out (for chemical reasons, samples were pooled prior to analysis), it is evident that fluorine levels in the dental tissues studied parallel corresponding levels in local water supplies.

The presence of lead in teeth has excited considerable interest in the past ten years on the supposition that lead levels in dental hard tissues may provide a reliable measure of lead ingestion. Pfrieme (1934) was among the first to show elevated lead levels in teeth of victims of lead poisoning (though he failed to find any difference between teeth from urban and rural environments) and later, Campbell et al (1950) established a relationship between soil and tooth lead levels in a study of disseminated sclerosis. Wyss (1951) established that the lead in teeth of metal workers was above normal. More recently, lead levels in deciduous teeth have been the subject of scrutiny. These studies have tended to focus on the effect on lead levels of environmental variables related principally to place of residence. Needleman and Shapiro and their co-workers, in an extensive series of studies [de la Burde and Shapiro (1975); Needleman and 
Shapiro (1974b); Needleman et al (1974a); Needleman et al (1972); Needleman and Shapiro (1973); Shapiro et al (1975); and Shapiro et al (1973)] have studied lead levels in various dental hard tissues in relation to housing (old or new) and pica, public schools attended (and thereby residence over small geographical areas), urban/rural classification, industrial development (primitive vs. industrialized population groups), and other residence-related variables. Needleman et al (1972) report a significant difference in lead content of teeth from children of the "lead belt" of Philadelphia (arithmetic mean $51.1 \mathrm{\mu g} / \mathrm{g}$ ) and suburban controls (arithmetic mean $11.1 \mu \mathrm{g} / \mathrm{g}$ )--the test was applied to log-transformed data to correct for skewness $(\alpha=0.01)$. Shapiro et al (1973) report similar results involving analyses of circumpulpal dentine and dentine from children of suburban Boston and Iceland. Perhaps most striking; however, are results reported by Shapiro et al (1975) on lead levels in the secondary dentine of teeth from contemporary urban industrialized and preindustrialized populations and from two prehistoric populations. From the absence of detectable levels of lead in a number of contemporary and ancient teeth from preindustrialized populations and the large difference in mean levels observed, particularly between teeth of their urban industrial population (urban Philadelphia) and teeth of contemporary jungle-dwelling preindustrialized populations (Mexican Lacandon and Tzeltales Indians), they conclude that the presence of lead in teeth reflects environmental lead levels to which populations are exposed. In studies involving circumpulpal dentine from teeth of children from two Philadelphia school districts, Needleman and Shapiro (1974b) and Needleman et al (1974a) describe the variation in lead levels over small geographic areas and suggest that both paint and airborne lead injestion are important variables in determining lead levels in teeth. In essentially the same type of study, Lockeretz (1975) describes variations in lead levels of whole 
teeth. from children resident in five different areas in st. Louis. While lead levels were clearly elevated $(\alpha=0.001 ;$ log-transformed data) for areas known to have lead paint problems, no differences were found between areas differing only in traffic density and/or the presence or absence of industrial sources of (airborne) lead. Rytömaa and Tuompo (1974) have also examined the relationship between lead levels in enamel, dentine, and whole teeth and urban and suburban. environments in Finland. While the data given do not permit tests of significance, the results generally reflect values obtained by Needleman et al (1972) for whole teeth: Stewart. (1974) has also examined lead levels in deciduous teeth of children aged 3-10 for three regions of Ireland characterized as urban, suburban, and rural. He found that urban teeth contained significantly more lead $(\alpha=0.05$; log-transformed datal than rural teeth for all age groups, while for suburban teeth, the differences were significant only at ages 3, 4, and 6 years. Suburban teeth in turn showed significantly greater lead levels than rural teeth at ages $4,5,8$, and 10 years. We have supplemented this analysis with our own, as follows: First, three separate regression analyses were performed, one each for urban, suburban, and rural teeth with age as the independent variable. Simultaneous, 95\% confidence intervals were constructed for the three possible differences in slopes-all of these intervals included zero. As a result, we felt that an analysis of covariance with age as the single covariate would be the most appropriate framework for analyzing stewarts' data, the hypothesis of interest being equality of the three additive factors for urban, suburban, and rural lead. This hypothesis was rejected at a significance level of 5\%. Further analysis revealed that the additive factors for urban and suburban teeth were significantly greater than for rural teeth. The urban and suburban factors were not significantly different $(\alpha=0.05)$, however. Habercam et al (1974), however, report little correlation 
between dental lead levels and the environmental variables, soil lead and residence lead, in a study of 18 pre-adolescent children from South Carolina. (Neither was substantial correlation between dental lead and age found.)

In 1959 , Hadjimarkos and Bonhorst suggested that it would be of interest to determine the selenium content of enamel and dentine of teeth from individuals residing in different regions; particularly seleniferous locations. They had already determined the selenium content of enamel and dentine from individuals of life-long residence in Portland, Oregon, and from a number of individuals of unknown residence history [Hadjimarkos and Bonhorst (1959)] and found that dentine levels from individuals of unknown residence history were generally lower than those from individuals known to have been lifelong residents of Portland. Differences were also found in the Se content of ancient and contemporary Greek teeth [Hadjimarkos and Bonhorst (1962)]. In view of the known cariogenic effect of selenium [Hadjimarkos $(1956,1969,1971,1973)$ ], it is surprising that it was not until 1973 that such a study was published. Suchkov et al (1973) describe results on caries incidence and the selenium content of teeth among residents of three natural geographic zones of the Soviet Chernovitsi region. The 177 teeth analyzed for se content were stratified by geographic zone, revealing differences between zones that reflect differences in the Se content of the soils in the zones. Unfortunately, the differences were not tested for significance and the number of specimens from each zone not given. However, the differences are sufficiently large in relation to tabulated estimates of variability within zones as to suggest that regional differences are indeed significant. Nixon and Myers (1970) have published analyses for se of enamel from three teeth each from Edinburgh and Manchester; there is no significant regional difference, however. 
Strontium levels in dental hard tissues also show variability with respect to geography. Steadman et al (1958) published the first evidence of this type involving $\mathrm{Sr}$ levels in successive layers of enamel and root tissue from residents of six different areas of the United States, two communities on Greenland, two Pacific islands, and from 5,000-year-old Indian teeth from Kentucky. While statements regarding the significance of the observed differences between regions could not be made, the evidence was suggestive. In particular, samples from the Pacific Island of Tonga had Sr levels greatly in excess of levels from other regions studied. Stronger evidence on geographic variation of $\mathrm{Sr}$ levels in teeth has been described by Gedalia et al (1973) based on a study of ashed fetal teeth from three regions with varying $\mathrm{Sr}$ levels in drinking water supplies. Their tabulated data, stratified by region and months in utero, are sufficient for tests. of significance for regional differences, though they report none. Our analysis, following removal of stratification with respect to months in utero, reveals that $\mathrm{Sr}$ levels in teeth from Region I (1.12 ppm Sr drinking water) are significantly different from levels recorded for Region III (1.53 ppm Sr drinking water), and that Sr levels in teeth from Region II ( $1.07 \mathrm{ppm}$ Sr drinking water) are significantly different from levels recorded for Region III, all differences being significant at $0.5 \%$ in the simultaneous testing sense. The sample estimate of correlation between mean $\mathrm{Sr}$ levels in teeth from these regions and the corresponding drinking water content is 0.9998. Kubota et a1 (1974) have also measured Sr levels in whole teeth from several regions in the U.S. and have found considerable variation between regions. Losee et al (1971) have observed similar variation between regions for $\mathrm{Sr}$. In particular, teeth obtained from residents of Fort Recovery and Delphos, Ohio, communities situated over extensive celestite $\left(\mathrm{SrSO}_{4}\right)$ deposits, contained approximately six times the $\mathrm{Sr}$ found in teeth from other regions. 
Interest in the ${ }^{90} \mathrm{Sr}$ content of calcified tissues has led to. investigations of the geographic variation of the ${ }^{90} \mathrm{Sr}$ content of teeth, primarily of deciduous teeth from individuals born in the mid-fifties to mid-sixties. Aarkrog (1971), for example, reports on the results of analyses of 235 whole crowns from children born in the period 1953-63 in Denmark, the Faroes, and Greenland. In an analysis of variance (a three-factor model including all higher-order interactions and using log-transformed data), area and year of birth were the significant main effects (at $5 \%$ ), with ${ }^{90} \mathrm{Sr}$ levels in Faroese teeth approximately four times as high as the Danish. Marei and Borisov (1972) report that ${ }^{90} \mathrm{Sr}$ levels in teeth from individuals living along the Siberian border were markedly higher than those. found for the remainder of the Soviet Union, while Lerch et al (1970) found no significant regional variation in the ${ }^{90} \mathrm{Sr}$ content of deciduous teeth from children resident in the principal towns of Switzerland.

Outbreaks of 'ouch-ouch' disease, believed by some to be caused by chronic cadmium toxication [JJ(1977)], have been studied in relation to Cd levels in dental hard tissues by Iwakura (1972). Whereas Kaneko et al (1974), in work cited earlier, did not find above-normal levels of $\mathrm{Cd}$ in teeth from a region known to be cadmium contaminated (Annaka, Japan), Iwakura does find such a relationship for teeth obtained from residents of a cadmium contaminated region in Toyama Prefecture, Japan. He reports significantly $(\alpha=0.05)$ higher Cd levels in enamel and dentine from teeth of this region when compared with data from an uncontaminated (unnamed) control region.

Incidental data are available bearing on the geographic dependence of barium in teeth from a study by Healy (1968) dealing with the Ba content of teeth of twin sheep raised on pastures of differing $\mathrm{Ba}$ content. The approximately sixfold difference in the barium content of the pasture was reflected in similar 
differences in the content of enamel, dentine, and whole crowns of the sheep. No similar data are available on human teeth.

Curzon et al (1971), in a study of environmental effects of molybdenum on caries, find that for regions of California of known molybdenosis, enamel samples from children were generally higher in Mo than samples taken from low Mo towns. The data, though scanty, are suggestive.

The concentration of zinc in enamel and dentine from teeth obtained from residents of four communities in the U.S., from Greenland, from two Pacific islands, and from two prehistoric Indian populations of the U.S. has been described by Brudevold et al (1963). While tests of significance involving regional variation were not possible, extremely low $\mathrm{Zn}$ levels in dentine and enamel obtained from Greenland were noted. The data are again suggestive but not conclusive.

Finally, Storozheva (1963) has reported that the tin content of teeth is greatily dependent on the environment in which subjects lived; and Losee and Little (1973) summarize emission spectrographic analyses for boron in enamel samples from 93 individuals of lifelong residence in 26 communities in 11 states of the U.S.: and spark source mass spectrometric analyses for the same element in enamel from 28 individuals of lifelong residence in 24 communities in 16 states of the U.S. (The same data are also published in Losee et al (1974a, b)). They conclude that $B$ must also be added to the list of elements whose concentrations vary on a geographic basis, although they do not tabulate their results by region. Evidence on geographic variation of the trace element content of dental hard tissues has been described above: Included were some results which suggest, at least for some elements, that regional variation is minor. Negative results on geographic variation have also been obtained for vanadium by Curzon et al (1974). 
In an analysis of 140 enamel samples from lifelong residents of 50 widely varying communities in the U.S., no relationship was found between an individual's residence and $V$ concentration. Nor do the data obtained by Shimada (1971) on the manganese content of 140 teeth from the Kagawa district and 191 teeth from Kanazawa, Japan, reveal significant regional differences. Rasmussen (1974) has also described negative results for $\mathrm{As}, \mathrm{Br}, \mathrm{Hg}$, and $\mathrm{Sb}$ concentrations in 52 ancient and contemporary Danish teeth. For the ancient teeth (from five cultural periods), no pattern in the results emerged which would permit the samples to be grouped according to place or period of origin, nor did contemporary teeth differ markedly from ancient teeth (only overall ranges for each element have been published for each cultural period).

A general critique of the evidence just surveyed follows. Our purpose is not to bring any individual conclusion into doubt; instead we hope to stimulate discussion of these results.

Variation of the inorganic composition of dental hard tissues with the geographic provenance of samples, though a simple concept, is difficult to deal with quantitatively. This difficulty originates in part from the arbitrary nature of boundaries that might be used to separate regions. Suppose, for example, that a simple random sample of teeth is drawn from a large population and that the data are then post-stratified by region for a number of moreor-1ess arbitrarily defined regions. Confidence intervals are then constructed which compare and contrast the concentrations of each element measured between regions: Assume for argument that all of these intervals contain zero and hence that the inference is made that there are no significant regional differences in the inorganic composition of dental hard tissues. If the data are then post-stratified a second time, employing a different set of perhaps equally acceptable regional boundaries, one may find that one or more of the new confidence intervals does not contain zero and hence that there are significant 
regional differences in the composition of dental tissues. One is left in doubt about the meaning of such results. In statistical parlance, the confidence intervals or equivalent hypothesis tests are conditional: They are conditional on (a priori) definition of regional boundaries. There is no problem as long as the regions defined have meaning in the overall framework of a particular investigation; however, statements regarding the presence or absence of significant regional differences in the inorganic composition of dental hard tissues are in themselves ambiguous, and this should be kept in mind.

In the example just given, reference is made to a random sample of dental specimens drawn from a target population. In studies involving the trace element analysis of dental specimens, both the (realistic) definition of this population and the procurement of a random sample of specimens from it are not trivial problems. This question has been addressed in very few investigations. Derise (1973) and Derise et al (1974a, b) obtained 175 permanent caries-free teeth "through the cooperation of dentists, randomly selected, throughout the State of Virginia," while Shapiro et al (1972) obtained deciduous and permanent teeth, collected at random, from the clinics of the School of Dental Medicine of the University of Pennsylvania. Cruickshank (1949), on the other hand, regarded the specimens he obtained through the Public Dental Clinic at Cambridge (U.K.) as a "random sample from the pre-adolescent secondary school population, biased only in the sense that all had a degree of 'overcrowded' dentition calling for orthodontic extraction." Even in two of these examples, one is left to speculate about the characteristics of the sampled population. Nevertheless, a sample of dental specimens, however obtained, can be viewed as a simple random sample drawn from some population with unknown characteristics. This applies 
to most investigations. If, on the basis of other evidence, one can infer that this population does not deviate significantly from the target population (the population from which one would have drawn a random sample if one could have) with respect to characteristics that influence trace element levels in teeth, then sample means and variances will be unbiased estimates of population parameters.

With few exceptions, the null hypothesic $H_{0}: \mu(\operatorname{Region} 1)-\mu(\operatorname{Region} 2)=0$, has been tested with the univariate t-statistic. Where only two regions are being compared for a single constituent of teeth, or where regions are being compared arbitrarily for pre-selected constituents, this is a meaningful procedure providing the usual assumptions (randomess, normality, etc.) are at least approximately correct. Where more than two regions are being compared simultaneously with respect to one or more inorganic constituents, however, the simple t-statistic yields confidence intervals that are too narrow: This is a problem in simultaneous inference, referred to earlier, which we will illustrate below with the data of Curzon et al (1975) and Kaneko (1972).

A general formulation of confidence intervals for regional differences for the type of confidence interval considered here is

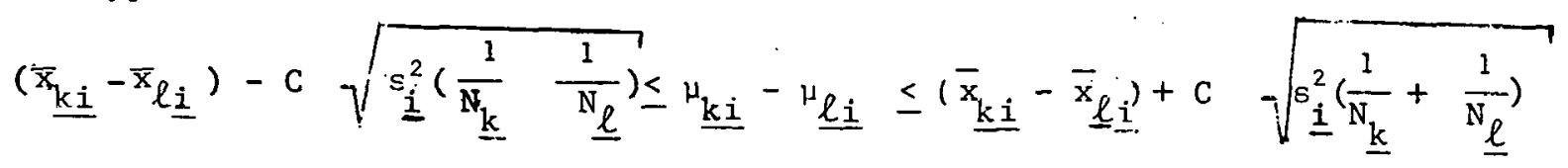

where the indices $\underline{k}$ and $\underline{\ell}$ refer to regions $\underline{k}$ and $\underline{\ell}$, and the index $\underline{i}$ refers to element $\underline{i} . \quad N_{k}$ and $N_{\ell}$ are the number of samples from regions $\underline{k}$ and $\underline{\ell}, \bar{x}_{\underline{k}}$ and $\bar{x}_{l_{1}}$, the corresponding sample means for the two regions for the $i$-th element, $s_{\underline{i}}^{2}$ the pooled sample estimate of variance for element $\underline{i}$, and $\dot{C}$ a number which determines the probability that the interval includes $\mu_{k i}-\mu_{k} \underline{l}$. 
For single element studies involving two regions, $C$ is students' $\underline{t}$ with $\mathrm{N}_{1}+\mathrm{N}_{2}-2$ degrees of freedom: For single element studies involving $\underline{r}$ regions, $C$ is the square root of $(\underline{r}-1) F_{\underline{r}}-1, N-\underline{r}$, where $F_{\underline{r}-1, N-r}$ is the F-Statistic with $\underline{r}-1$ and $N-\underline{r}$ degrees of freedom and $N$ is $\mathrm{N}_{1}+\mathrm{N}_{2}+\ldots+\mathrm{N}_{\underline{r}}$, the total number of samples [Scheffe(1959)]. The expressions for $\mathrm{C}$ become more complex in multielement studies. With one exception, the examples below are based on the 'greatest-root' distribution [Morrison (1976)].

Recall that Kaneko (1972) and Kaneko et al (1974) measured the concentrations of five elements in 99 teeth from four regions in Japan. Though some elements were not detected (or measured) in all 99 specimens, we will assume that they were. The univariate $\underline{t}$ statistic employed by Kaneko is approximately 2 for the $95 \%$ confidence interval with 97 degrees of freedom. $\mathrm{C}$, however, based on the 'greatest-root' distribution is approximately 4.3 for confidence intervals equal to or larger than $95 \%$. In other words, these simultaneous confidence intervals for comparisons among four regions for the five elements are approximately twice as broad as those obtained with $\underline{t}$ and, t: under these circumstances, several regional differences judged to be significant (with $\underline{t}$ ) lose their significance, while the significance of several other regional differences is degraded.

Next, recall that Curzon et al (1974) compared the concentrations of 25 elements in 36 teeth from Dunedin, New Zealand (the teeth were derived from 18 individuals, though for argument we will assume them to be independent), with 56 teeth from the United States. As above, we assume that each element was detected in all 92 teeth. The univariate $\underline{t}$ statistic employed by curzon et al is approximately 2 for the $95 \%$ confidence interval with 90 degrees of freedom. $C$, however, based on the 'greatest-root' distribution (in this case, proportional 
to the square root of the F-statistic with 25 and 66 degrees of freedom and yielding confidence intervals equal to or larger than $95 \%$ ) is 7.6 . Based on these simultaneous confidence intervals for two regions and 25 elements, none of previously detected significant differences are significant. Bonferroni intervals [Morrison (1976)], which are equally valid, are considerably narrower and hence preferable, however. C, obtained from tables reproduced by Miller (1966), is approximately 3.2. With this value, mean levels of six of the 25 elements studied are significantly different in the two populations, thus illustrating an important point: When confidence intervals can be constructed in more than one way for the same inequality involving the probability of Type I error, the procedure yielding the narrowest intervals is preferable [Miller(1966)]. To conclude, most investigations of the geographic dependence of the inorganic composition of dental hard tissues are not free of defect. This is less a shortcoming of these studies than it is a comment on the very real difficulties encountered in this field. 


\section{$\underline{2.4} \underline{\underline{\mathrm{Age}}}$}

The inorganic composition of dental hard tissues has been investigated with respect to a number of variables in addition to sample provenance. Among these are age, sex, tissue type, occupation, and various health-related variables. In several cases, the question of inorganic composition has also been examined to determine whether type of tooth is a significant variable and how variation in the composition of teeth from the same mouth compares to variability between individuals.

Among multi-element studies, the investigation by Crowell et al (1934) on the $\mathrm{Ca}, \mathrm{Mg}$, and $\mathrm{P}$ content of whole teeth is noteworthy in many respects. Not only were the variables age and sex examined, but the condition of the teeth (sound/carious), the type of tooth, and the variability in composition of teeth from the same mouth relative to variability between mouths were studied with respect to inorganic composition. Furthermore, 'known' (but unstated) statistical principles were employed to check their conclusions. These were that there were no differences in the $\mathrm{Ca}, \mathrm{Mg}$, and $\mathrm{P}$ content of teeth with respect to sex, condition of the teeth, type of tooth, and that there was little difference with respect to age. They also concluded that variation. in composition of teeth from the same mouth was as wide as for teeth from different mouths. More recently, the influence of age or the inorganic (multi-element) composition of teeth has been studied by Storozheva (1963) ( $\mathrm{Pb}, \mathrm{Sn}$ ), by Dmitriev (1967, 1969) ( $\mathrm{Ag}, \mathrm{Co}, \mathrm{Cr}, \mathrm{Cu}, \mathrm{Mn}, \mathrm{Ni}, \mathrm{Pb}$, Pt, Ti, Zn), by Zakson (1968) (Al, Cu, Fe, Mn, Mo, Pb, Ti, Zn), and by Derise (1973) and Derise et al (1974a, b) (Al, Ca, Cl, Co, Cu, F, Fe, I, K, Mg, Mn, Na, $\mathrm{P}, \mathrm{Pb}, \mathrm{Se}, \mathrm{Sr}, \mathrm{Zn})$. Storozheva (1963) found that $\mathrm{Pb}$ levels in permanent teeth increased with age and Dmitriev (1969) notes increases in $\mathrm{Cu}, \mathrm{Ni}, \mathrm{Pb}$, and $\mathrm{Zn}$ levels with age for persons aged 20 to 60 years. Zakson (1968), however, failed to find any change with age in $\mathrm{Cu}, \mathrm{Fe}, \mathrm{Mn}, \mathrm{Pb}, \mathrm{Ti}$, and $\mathrm{Zn}$ levels in teeth from persons in the upper 
age categories $51-60,61-70$, and $71-80$ years. He did find that Mo levels increased and that Al levels decreased with age. In determining the significance of age for her results, Derise (1973) employed univariate analysis of variance for each of the 17 elements studied: Two models, one involving two main effects (age and sex) and one two-factor interaction (age $\mathrm{x}$ sex), the second involving three main effects (age, sex, and tissue type), three two-factor interactions (age $x$ sex, age $x$ tissue type, and sex $x$ tissue type), and one three-factor interaction (age $x$ sex $x$ tissue type), were used. The first model, results from which are the basis of the following comments, was applied separately to enamel and dentine. Where the sex and age interaction is insignificant (for all but $\mathrm{Zn}$-dentine levels), the hypothesis of no age and/or sexual differences has meaning. Significant differences between the four age groups formed from the data (10-12,13-16, 17-24, $\geq 25$ years) were found for dentine $(\mathrm{Al}, \mathrm{Ca}, \mathrm{Cl}, \mathrm{Co}, \mathrm{F}, \mathrm{I}, \mathrm{Mg}, \mathrm{Mn}, \mathrm{P}, \mathrm{Pb})^{*}$ and for enamel ( $\mathrm{Al}, \mathrm{Co}, \mathrm{Cu}, \mathrm{F}, \mathrm{K}$, $\mathrm{Mn}, \mathrm{P}, \mathrm{Pb}, \mathrm{Se}, \mathrm{Sr}, \mathrm{Zn})$. Age categories which were significantly different were then identified through Duncan's multiple range test [Miller (1966)], though its application should be limited to balanced designs (equal numbers of individuals in each agecategory and equal numbers of males and females within each age-category). The results of this test suggest significant differences between two or more age categories for all elements for both enamel and dentine $(\alpha=0.05)$, however. While the evidence presented by Derise demonstrating significant diffezences Detroen age grouns is =0itd (the probability is only 0.006 that she would have observed by chance alone, eight cases out of 34 possible element-tissue type combinations where concentrations are strictly increasing or decreasing among the four age categories), the differences are minimal. Nowhere do any age categories differ by more than a factor of 2.1 , and in most cases, the differences are less than a factor of 1.4. There is little doubt that had multivariate statistical methods been used to analyze her data,

* Zn was also said to be significantly affected by age, but the presence of a significant interaction in this case would normally require separate evaluation of differences among age categories for each sex. [Scheffe (1959)]. 
Derise would have found less significant differences between age-categories for some elements. (Inasmuch as correlation between elements and between enamel and dentine levels is probably significant; at least. for some elements, the series of univariate hypothesis tests that were constructed are interrelated. Furthermore associated multivariate critical points/confidence intervals would probably be larger/wider in keeping with the simultaneous nature of the inferences made.)

Incidental data bearing on the relationship between age and the concentrations of $\mathrm{La}, \mathrm{Sm}, \mathrm{Tb}$, and $\mathrm{Yb}$ in enamel and dentine are tabulated by Steinnes et a1 (1974); however, in view of the limited quantity of data, statistical analysis is unwarranted. There are no immediately apparent trends in the data.

The variation in the concentration of single elements in dental hard tissue with respect to age has been the subject of numerous investigations. By element, these include -- As [Vikhm (1963)], Cu [Brudevold and Steadman (1955)], F [McClure and Likins (1951); Brudevold et al (1956c); Jackson and Weidmann (1959); Yoon et al (1960)], Mn [Battistone et aI (1967)], Pb [Pfrieme (1934); Maulbetsch and Rutishauser (1936); Brudevold and Steadman (1956a); Strehlow and Kneip (1969); Strehlow (1972); Habercam et al (1974); Malik and Fremlin (1974); Stewart (1974)], Pb-210 [Lanzola and Allegrini (1973)], Se [Hadjimarkos and Bonhorst (1959); Mamedova (1965); Suchkov et al (1973)], Sn [Brudevold and Steadman (1956b)], Sr [Steadman et al (1958); Gedalia et al (1973)], ${ }^{90} \mathrm{Sr}$ [Butler (1961); Lerch et al (1970); Carach et al (1970); Aarkrog (1971); Rytbmaa (1971); Marei et al (1972); Prokofiev et al (1973)], and 2n [Cruickshank (1949); Lazarev (1962); Brudevold et al (1963)]. Also of interest are data gathered by Nixon and co-workers: Age, though not investigated explicitly, has been incorporated into their tabulation of analytical results for individual samples for Cu [Nixon and Smith (1962)], Hg [Nixon et al (1965)], Mn [Nixon et al (1966)], Sb [Nixon et al (1967a)], and Zn [Nixon et al (1967b)]. 
Brudevold and Steadman (1955) found no apparent relationship between the $\mathrm{Cu}$ content of enamel and the age of the donor. This result is corroborated by the data tabulated by Nixon and Smith (1962) on the $\mathrm{Cu}$ content of 39 samples of inner and outer enamel from teeth of individuals of known age and sex. This conclusion is based on the following analysis by us of Nixon's data -- (i) A histogram of the results of 100 analyses [Nixon and Smith (1962)] suggests that the data are approximately log-normally distributed, hence our analysis is in terms of logtransformed data; (ii). Since two responses ( $\mathrm{Cu}$ levels in inner and outer enamel) are measured for each experimental unit (tooth), and since one cannot rule out correlation between errors in $\mathrm{Cu}$ levels in inner and outer enamel, a multivariate statistical model is employed; and (iii), In order to account for the effect of both age and sex, the model employed is a one-way multivariate analysis of covariance model with sex as the additive main effect and with age as the single (linear) covariate: The effect of age is assumed the same for males and females. The appropriateness of treating age linearly will be discussed later. The model, therefore, is given by equation (14) and the hypothesis tested here is that of no age effect ( $1 . e .$, no regression relationship). At the $5 \%$ level of significance this hypothesis cannot be rejected and we conclude that the data do not support the existence of a significant age-effect for this model. The conclusion applies simultaneously to inner and outer enamel.

Fluorine levels have been shown to increase somewhat with age in the outer layers of enamel and to remain approximately constant in inner enamel by Brudevold et al (1956c). Unfortunately, the histories of individual teeth were not known, and the well-documented relationship between drinking water $F$ and enamel $F$ may have confounded their results. McClure and Likins (1951) were unable to find significant variation with age of $F$ levels in enamel and dentine from samples of known provenance, though as mentioned previously, the broad range of age groups used to categorize 
their data may have obscured any real variation: If examined in detail, however, both of these studies are not inconsistent with the findings of Jackson and Weidmann (1959) demonstrating that fluorine accumulation in bulk enamel and dentine tends to level off after a period of time-that:depends" on the fluorine content of local drinking water. A relationship which describes these results (mathematically) was advanced and discussed in the previous section. 'Experimental results of a similar nature on $F$ levels in enamel and dentine have been described by Yoon et al (1960).

The only results available on the possible effect of age on $\mathrm{Hg}$ levels in teeth derive from the tabulation of data by Nixon et al (1965) on anaiyses of 28 samples of inner and outer enamel from individuals of known age. As in the case of $\mathrm{Cu}$, histograms of the data published by Nixon et al. (1965) suggest a log-normal distribution; hence our analysis is in terms of $10 \mathrm{~g}$-transformed data. The multivariate statistical model employed in this case, however, is a multivariate linear regression model with age as the single independent variable (equation (15)) and the hypothesis tested is that of no age effect (i.e., no regression relationsh1p). At the $5 \%$ level of significance, this hypothesis cannot be rejected and therefore we conclude that the data for $\mathrm{Hg}$ do not support the existence of a significant age-effect simultaneously for inner and outer enamel.

For females aged 17-30, Battistone et al (1967) found Mn levels in the enamel and dentine of erupted teeth that were significantly lower than for females aged 8-16, although significant differences between the same age categories were not found for male donors. We have supplemented this analysis with our own, based on the data on both erupted and unerupted enameliand dentine that are compiled by Battistone. Many of the same considerations discussed above apply to this data. For example, Nixon et al (1967c) plot histograms for Mn analyses of 62 enamel samples that suggest Mn concentrations are approximately log-normally distributed; hence our statistical analysis is in terms of log-transformed data. Inasmuch as 
Battistone et al (1967) provide information on the sex of donors of enamel and dentine as well as their age, our analysis allows for specific differences between sexes. In addition, because the effect of age uncovered by Battistone appears to be sex-specific, our model incorporates a separate linear age term for each sex. Hence, the multivariate model employed is given by equation (16) and the hypothesis tested involves simultaneous tests against zero of the coefficient of donor age for male and female enamel, and for male and female dentine. For both erupted and unerupted teeth, this hypothesis cannot be rejected at a 5\% significance level: We conclude, therefore, that the data compiled by Battistone do not support the existence of a significant age-effect. (Nor do the data support the existence of male and female age-effects that are significantly different from one-another). Because this is a multivariate test and because log-transformed data were used, direct comparison with Battistone's inference is not possible: We have observed, however, that the existence of a significant age-effect for erupted female dentine and enamel is sensitive (in a univariate one-way analysis of vartance) to definition of age-categories. Nixon et al (1966) have also compiled data on Mn levels in dental hard tissues; the data include Mn levels in occlusal enamel, the inner and outer layers of enamel from the gingival third, and the inner and outer layers of enamel from the middle third of teeth of known type from donors of known age and sex. For this data, we have adopted a model which is the same as that employed for $\mathrm{Cu}$-- the added information on tooth type cannot be utilized effectively in view of the limited number of analyses that are tabulated; tooth type is thus assumed to be without effect. In addition, the linear coefficient of age is assumed independent of sex (viz. the analysis of Battistone et al (1967)'s data). Hence the model is a multivariate one-way analysis of covariance model with age as the single covariate; 
it involves five responses and two levels in the main effect (sex). Employing log-transformed data, the hypothesis of no age effect cannot be rejected at a significance level of $5 \%$. Hence, the data do not support the existence of a significant age-effect simultaneously for the five types of enamel studied.

The effect of age on $\mathrm{Pb}$ levels in dental hard tissues has been extensively studied. Maulbetsch and Rutishauser (1936) noted that lead levels in teeth are unaffected by age, whereas earlier Pfrieme (1934) found that they increase with age. More recently, Brudevold and Steadman (1956a) found that lead levels in enamel tend to increase with the age of the donor: $\mathrm{Pb}$ in enamel from individuals over 50 years of age was approximately twice levels found for those under 20 years of age. This result was extended to cover lead levels in whole teeth by Strehlow and Kneip (1969), where a plot of the midpoint of each of six age groups against the logarithm of the mean lead content of ashed whole teeth for each group shows good linearity. The corresponding regression equation (obtained by us) is

$$
\text { In } \mathrm{Pb}(\mathrm{ppm})=0.0390 \times \text { age }+2.5990
$$

Similar results are reported by Stewart (1974) for deciduous teeth of children aged 3-10. Curves of $\log \mathrm{Pb}$ vs, age (analyzed by analysis of covariance earlier in this paper) also show good linearity if sample provenance is properly accounted for. Slopes of curves for children from urban, suburban, and rural environments are not significantly different, however. On the other hand, Habercam et al (1974), report finding little correlation between age and the lead content of teeth from 18 pre-adolescent children from South Carolina, and the relationship between age and $\mathrm{Pb}$ described by Malik and Fremlin (1974), though showing that lead levels tend to increase with age in both enamel and dentine, is weak (their data are too limited for multivariate linear regression analysis and associated hypothesis testing on the significance of an age-effect). 
Nixon et al (1967a) have tabulated analyses for antimony for 10 individuals aged 14-61. The data include analyses of occlusal enamel, the inner and outer layers of enamel from the middle third of teeth of known type from donors of known age and sex. Unfortunately, there are not sufficient data for use with multivariate statistical models of the type considered above. Separate hypothesis tests for each enamel type based on univariate analysis of covariance (sex as the main effect, age as the covariate) failed to reveal any slgnificant age-effect, however (we employed log-transformed data in these analyses).

Some evidence exists regarding the effect of age on selenium levels in teeth. Hadjimarkos and Bonhorst (1959) could find no apparent trends in the Se content of enamel and dentine when four age groups were found from their data. Likewise, the variation in Se levels of teeth as a function of age reported by Mamedova (1965) is erratic. Data published by Suchkov et al (1973), however, suggest that Se levels increase with age if proper provision is made for the residence of the donor.

Brudevold and Steadman (1956 b) describe data which, though quite limited, tend to show that tin levels increase with time in the outer layers of ename1. Much higher tin concentrations were recorded for enamel from restored teeth, however, suggesting that contamination of enamel from $\mathrm{Sn}$ present in amalgam fillings may have been important.

The concentrations of $\mathrm{Sr}$ found in enamel from teeth of donors resident in the same community shows little variation with age [Steadman et al (1958)], and the variable, months in utero, has little relationship to $\mathrm{Sr}$ levels in ashed fetal teeth [Gedalia et al (1973)]. Furthermore, Sr levels in erupted and unerupted third molars, obtained from the same mouth, are not significantly different indicating that, at least, post eruptive uptake is minimal [Gedalia et al (1973)].${ }^{90} \mathrm{Sr}$ levels, however, have been shown to vary markedly with the age of the donor and, 
perhaps more significantly, with the year of extraction. Butler (1961), for example, has found that the ${ }^{90} \mathrm{Sr}$ content of teeth (deciduous and permanent) collected in 1959 varied markedly with the age of the tooth, the youngest teeth (5 years) being approxtmately six times as high in ${ }^{90} \mathrm{Sr}$ as the oldest teeth (20-35 yrs). Carach et al (1970) report similar results for deciduous and permanent teeth from selected population groups of Slovakia, as does Rytymaa (1971) for deciduous teeth collected in Helsinki during the period 1956-1963, and Prokofiev et al (1973) for Soviet teeth. Aarkrog (1971), however, has found that ${ }^{90} \mathrm{Sr}$ levels in deciduous teeth collected in Denmark and the Faroes during 1962-65 and 1966-69 peak for donors born about 1964: Post-1967 levels are approximately the same as those found for donors born around 1960. A linear relationship between the ${ }^{90} \mathrm{Sr}$ content of teeth and fall-out rates (specifically, the mean fall-out rate in the year of birth and the following year, the fall-out rate in the year prior to birth, and the accumulated fall-out by the end of the year of birth) was found to be highly significant (i.e., its abllity to reproduce the data was excellent). Data on the ${ }^{90} \mathrm{Sr}$ content of deciduous teeth collected in Switzerland in separate campaigns in 1961, 1963, 1964, 1966, 1967, and 1968 have been compiled by Lerch et al (1970). These authors find that in addition to age, year of collection is related to the measured ${ }^{90} \mathrm{Sr}$ content of teeth. Their data are plotted in Figure 1 as a surface. The $x$ - and $y$-axes are year of birth and age at time of extraction (year of collection is the sum of year of birth and age). Circled intersections on the grid are measured values; all other intersections are based on interpolation or extrapolation of measured data. The variation in ${ }^{90} \mathrm{Sr}$ levels on the surface is fairly uniform (the surface is smooth), though subject to a variety of interpretation. Of considerable interest, however, is 


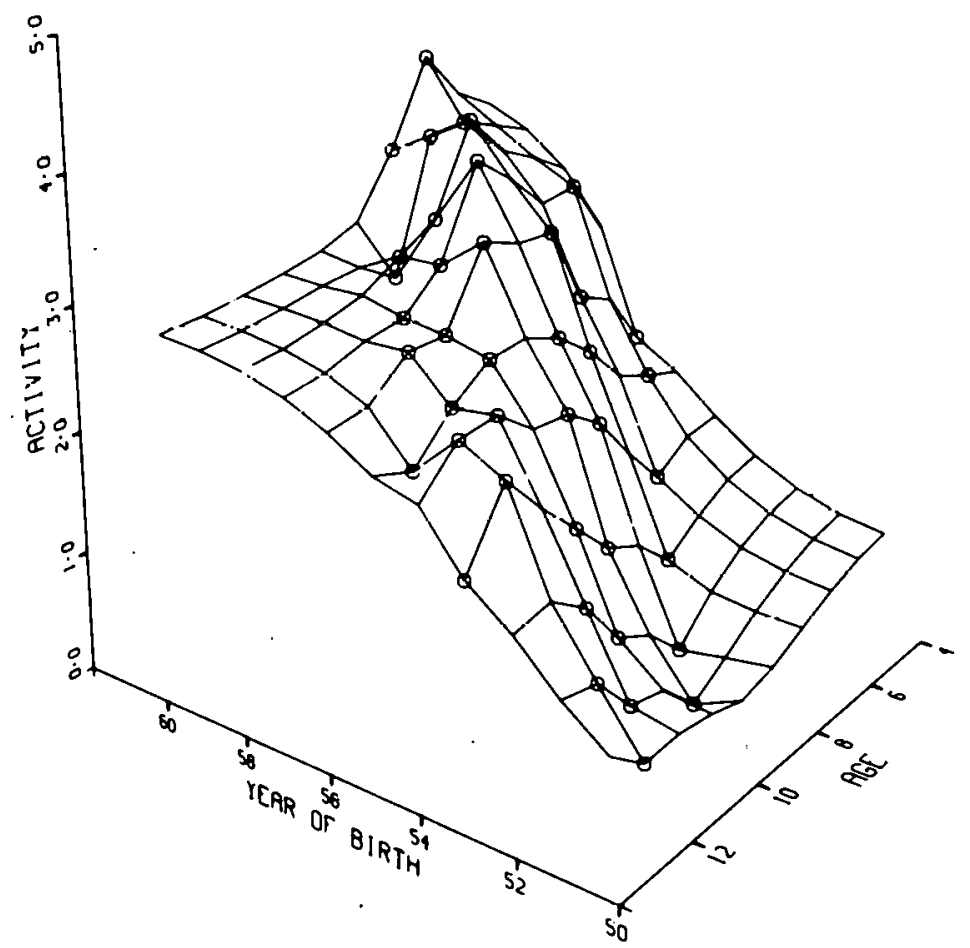

Figure 1. SR-90 content of deciduous teeth 
the fact that for constant year of birth, ${ }^{90} \mathrm{Sr}$ levels rise and then fall again as the age of the donor increases. We are tempted to suggest that ${ }^{90} \mathrm{Sr}$, once incorporated into the tooth, is capable of being leached out again (when, for example, environmental levels fall), but it is probable that the types of teeth on which data are based are different for younger and older individuals. Hence the age of the tooth (exposure time in the oral environment) is not adequately reflected by the age of the donor.

Cruickshank (1949) has measured the $\mathrm{Zn}$ content of ashed enamel and dentine derived from ca 200 sound premolars and has examined the effect of age, sex, position of the tooth, and the condition of the specimen on the resulting concentration data. He notes an increase of 12-15\% (from 179 to $216 \mathrm{ppm}$ ) in $\mathrm{Zn}$ levels over a seven-year period for subjects aged 10 to 17 years, though the difference is probably not significant (the standard errors are said to all lie between 20 and $30 \mathrm{ppm}$ ). Brudevold et al (1963) have also measured $\mathrm{zn}$ concentrations in enamel and dentine and, though no discernable trend with age was found, believe it reasonable that $\mathrm{Zn}$ is acquired throughout life. Masking of differences between age groups by individual differences in $\mathrm{Zn}$ ingestion, even for individuals residing in the same region, is advanced as a possible reason for failure to find a trend with age. Lazarev (1962) reports similar results (though his measured $\mathrm{Zn}$ levels differ radically from those reported above). Nixon et al (1967b) have compiled data on $\mathrm{Zn}$ levels in occlusal enamel, the inner and outer layers of enamel from the gingival third, and the inner and outer layers of enamel from the middle third of teeth of known type from donors of known sex and age. The multivariate statistical model in terms of which we have analyzed this data has been described above. Sex is the main effect and age is the single covariate: Log-transformed data have been used in view of the shape of histograms for $\mathrm{Zn}$ levels in dental hard tissues [Nixon et al $(1967 \mathrm{~b}, \mathrm{c})]$. The hypothesis of no 
age-effect cannot be rejected at a significance level of $5 \%$. For the five types of enamel studied, the data do not support the existence of significant age-effects.

We conclude this section now with some general comments on the adequacy of introducing age as a (linear) covariate in statistical analysis of log-transformed compositional data. In only three instances, $\mathrm{Pb}, \mathrm{F}$, and ${ }^{90} \mathrm{Sr}$ has a clearly defined and well-behaved pattem of variation in the elemental content of dental hard tissues with age been established. For $\mathrm{Pb}$, at least over a restricted span of years, the $\log$ of the $\mathrm{Pb}^{\circ}$ concentration is linearly related to age [Strehlow and Kneip (1969); Stewart (1974)] and increases with age. For F, the relationship is somewhat more complex (see above) [Jackson and Weidmann (1959)], but F levels do increase with age, though not indefinitely. For $\mathrm{Pb}$, therefore, introducing age as a covariate in data analysis is acceptable. Less is known about the adequacy of introducing age as a covariate for $F$ (not done here) and to a greater extent for other trace constituents in teeth: Indeed this is one of the purposes of statistical data analysis. For any trace constituent whose concentration is a monotonic function of age, the logarithm of the concentration will also be approximately a linear function of age for a restricted time span. Hence introduction of age as a covariate and testing of the coefficient of age against zero can be used to uncover a significant age-effect, though the hypothesis test is of course biased in the presence of any underlying deviation from the log-linear relationship. Non-monotonic dependence of concentration on age, especially if significant, will seriously affect a statistical analysis where age enters as a linear term (for ${ }^{90} \mathrm{Sr}$ ). Preliminary graphical analysis will usually reveal any serious departures from the assumed model, however. For the data analyzed above, no such departures have been detected, though the amount of data entering these analyses is quite limited. Our failure to find statistically significant age-effects is closely related to the amount of data 
available. For the sample sizes considered, an age-effect, to be significant, would be obvious from simple inspection of the data. Finally, we emphasize once again that the hypotheses tested above apply only to the data actually analyzed and do not extend to teeth or dental hard tissues in general. 


\section{$\underline{\underline{2.5}} \underline{\underline{\text { Sex }}}$}

The influence of sex on the inorganic composition of dental hard tissues has received some attention. The work of Crowell et al (1934) on the Ca, $\mathrm{Mg}$, and $\mathrm{P}$ content of whole teeth has already been mentioned. In addition, Söremark and Samsahl (1962a, b) and Söremárk and Lundberg (1964a, b) have measured the concentrations of 17 elements ( $\mathrm{Ag}, \mathrm{Au}, \mathrm{Br}, \mathrm{Ca}, \mathrm{Cl}$, $\mathrm{Co}, \mathrm{Cr}, \mathrm{Cu}, \mathrm{Fe}$, $\mathrm{Mn}, \mathrm{Na}, \widetilde{\mathrm{P}}, \mathrm{Pt}, \mathrm{Rb}, \mathrm{Sr}, \mathrm{Zn}, \mathrm{W}$ ) in normal human enamel and dentine and have found no significant variation with respect to sex; nor has Dmitriev (1969) found variation with respect to sex in the enamel concentrations of $\mathrm{Ag}, \mathrm{Co}, \mathrm{Cr}, \mathrm{Cu}$, $\mathrm{Mn}, \mathrm{Ni}, \mathrm{Pb}, \mathrm{Ti}$, and $\mathrm{Zn}$. Derise (1973) and Derise et a1 (1974a, b), however, have found some significant differences in the inorganic composition of enamel and dentine of specimens from male and female donors. Of the 17 elements studied ( $\mathrm{Al}, \mathrm{Ca}, \mathrm{Cl}, \mathrm{Co}, \mathrm{Cu}, \mathrm{F}, \mathrm{Fe}, \mathrm{I}, \mathrm{K}, \mathrm{Mg}, \mathrm{Mn}, \mathrm{Na}, \mathrm{P}, \mathrm{Pb}, \mathrm{Se}, \mathrm{Sr}, \mathrm{Zn}$ ), significant sex-related differences were found in the composition of enamel for Se and $\mathrm{Zn}$, and of dentine for $\mathrm{Ca}, \mathrm{I}$, and $\mathrm{Mg}$. These inferences are derived from the two-factor model and univariate statistical techniques reviewed earlier. The reported differences are smal1, however. The maximum difference observed was for I, where the content of female dentine was $26 \%$ above that for male dentine (based on overall male/female means). The most significant difference recorded, however, was for $\mathrm{Zn}$, but it amounts to only a $6 \%$ change in composition. Had multivariate statistical methods been employed by Derise, we suspect, but cannot confirm, that the sex-related differences would vanish (that they would no longer be significant).

Several single element studies that address sex-related differences of the inorganic composition of teeth have also been published. Battistone et al (1967), have found the enamel from unerupted male teeth to be significantly (23\%) lower in Mn than enamel from unerupted female teeth, while no difference was found for erupted teeth. Extending our analysis of this data, described in section 2.4, 
we find that the (multivariate) hypothesis of no sex-related effects cannot be rejected at a significance level of $5 \%$ for either erupted or unerupted teeth. In other words, for the multivariate model we adopted, the data do not support the existence of significant sex-related differences in the Mn content of enamel and dentine. The results of the analysis for Mn of 140 (whole) teeth from two districts in Japan, published by Shimada (1971), also fail to reveal. significant differences between male and female teeth within each of these regions.

No sex-related differences were found by Pfrieme (1934) in the $\mathrm{Pb}$ content of teeth, and Mamedova (1965) failed to find differences in the Se content of enamel with respect to sex. Variation in the $\mathrm{Zn}$ content of enamel and dentine of male and female subjects reported by Cruickshank (1949), given standard errors on the order of 20-30 ppm, is also without significance, although female dentine is consistently higher in $\mathrm{Zn}$ than male dentine (approximately $5 \%$ higher for normal and defective teeth, for upper and lower teeth, and for two of three tabulated age categories).

Although they report no specific analysis designed to reveal the effect of sex on the inorganic composition of enamel, Nixon and his co-workers have published data that permit some limited inferences about sex-related differences in the $\mathrm{Cu}$ [Nixon and Hamilton (1962)], Mn [Nixon et al (1966)], Sb [Nixon et al (1967a)], and $\mathrm{Zn}$ [Nixon et al (1967b)] content of enamel. The multivariate statistical models we have used to describe $\mathrm{Cu}, \mathrm{Mn}$, and $\mathrm{Zn}$ levels in enamel were introduced in section 2.4: It remains to test the (multivariate) hypothesis of no sex-related effects. For these elements, this hypothesis cannot be rejected at a significance level of $5 \%$. We conclude, therefore, that the data do not support the existence of significant sex differences in the concentrations of 
these elements for the types of enamel studied. For $\mathrm{Sb}$, as described earlier, Insufficient data exist for use with a multivariate model: However, no significant sex-related differences were found in separate univariate analyses of covariance (sex as main effect, age as covariate).

In general, we believe the existing data surveyed by us do not support the existence of significant sex-related differences in the inorganic composition of dental hard tissues. To find such differences, considerably more data is required. 


\section{$\underline{\underline{2.6}}$ Distribution}

The distribution of the inorganic constituents of teeth within individual $\therefore$ specimens is far. from uniform. Brudevold and Söremark (1967) have reviewed much of the evidence on the distribution of trace elements in enamel, most of which is derived from studies by Brudevold and Steadman and their co-workers \{ $\mathrm{Cu}$ [Brudevold and Steadman (1955)], F [Brudevold et al (1956c); Yoon et al (1960)], Pb [Brudevold and Steadman (1956a)], Sn [Brudevold and Steadman (1956.b)]; Sr [Steadman et al (1958)], Zn [Brudevold et. al (1963)], and a multi-element survey. [Steadman et al (1959)]\}, in which enamel samples from several specimens are pooled in order to provide sufficient material for analysis. Weatherell et al (1974) have reviewed much of the data on $F$ distributions in enamel. Since Brudevold's review, a number of investigations reporting on analyses of samples from individual specimens have been published, and several newer analytical techniques have been employed to map with some detail the distribution of single elements in individual specimens.

Besic et al (1968), for example, have analyzed sections of five teeth for $\mathrm{Ca}, \mathrm{C} 1, \mathrm{Mg}, \mathrm{Na}$, and $\mathrm{P}$ by electron probe microanalysis. Since areas a few microns in diameter could be analyzed, scans of one section from each specimen produced a profile showing the dependence of concentration on depth (from the external enamel, through the dentine-enamel junction (DEJ), to the pulpal-dentinal junction). While the number of specimens examined are too few to justify statistical analysis, the discontinuous change in concentration across the DEJ observed for most of the elements studied is noteworthy. Ahlberg and Akselsson (1976), in a demonstration of the feasibility of applying proton-induced X-ray emission techniques to the chemical assay of dental hard tissues, tabulate the concentrations of the macroconstituents $\mathrm{Ca}$ and $\mathrm{P}$, the microconstituents $\mathrm{Cl}, \mathrm{K}$, and $\mathrm{S}$, and the trace constituents $\mathrm{Br}, \mathrm{Cu}, \mathrm{Fe}, \mathrm{Mn}, \mathrm{Ni}, \mathrm{Pb}, \mathrm{Sr}$, and $\mathrm{Zn}$ found in the external and internal 
enamel, and the dentine and pulpal wall of dentine of a single specimen. With the exceptions of $\mathrm{Ca}, \mathrm{P}$, and $\mathrm{Sr}$, the external enamel and the pulpal wall contain higher concentrations than the internal enamel and dentine, respectively. Stubbins and Fremlin (1967) report on the charged particle activation analysis of sections of enamel from permanent, untreated adult teeth for $\mathrm{C} ; \mathrm{Ca}$, and $\mathrm{P}$. Autoradiography following activation was used to determine the distribution of these elements throughout each section. (This is, incidentally, the only direct determination of carbon in dental tissues we've found. Previous work has focused primarily on the $\mathrm{CO}_{2}$ content of dental hard tissues [cf Brudevold and Soremark (1967)].) The distribution of $\mathrm{C}$ in enamel, measured by charged particle activation analysis, parallels results based on $\mathrm{CO}_{2}$ analyses which show higher levels in internal enamel compared with external enamel.

In his work on the Al content of enamel, Dolinsek (1975) found that in the single specimen for which analyses were performed at several depths in enamel, the Al concentration generally decreased with increasing depth.

$\mathrm{Cu}$ has been assayed in inner and outer enamel from 100 teeth by Nixon and Smith (1962) and the results tabulated for 39 specimens. We have already considered this data in the context of sex and age-related effects and extend our analysis here to testing the hypothesis of equal response effects, i.e., whether there exists a significant difference in $\mathrm{Cu}$ levels in inner and outer ename1. This hypothesis is easily formulated and interpreted providing, as in this case, that the hypotheses of no age and sex-related effects cannot be rejected: At a significance level of $5 \%$, the hypothesis of equal response effects cannot be rejected and hence we conclude that the data do not support the existence of significant differences in the $\mathrm{Cu}$ content of inner and outer enamel, a result which agrees with the more qualitative evaluation by Brudevold and Steadman (1955). 
$\mathrm{Hg}$ has also been assayed in inner and outer enamel by Nixon and his co-workers [Nixon et al (1965)]: As for $\mathrm{Cu}$, our analysis of this data can be extended to test for equal $\mathrm{Hg}$ levels in inner and outer enamel. At a significance level of $5 \%$, this hypothesis cannot be rejected; hence, we conclude that the data do not support the existence of significant differences in the Hg content of inner and outer enamel. However, had a model (equation (13)) been adopted which ignored the effect of age on the data (recall that the age effect could not be regarded as significant with a model which incorporates age linearly), the hypothesis of equal response effects (Hg levels in inner and outer enamel) would be rejected.

Nixon et al (1966) have measured the Mn content of five types of enamel: Our analysis of this data, begun in section 2.4, can also be extended to cover the hypothesis of equal response effects; i.e., equal Mn levels in the five types of enamel studied. Interpretation of the hypothesis is facilitated by failure to reject hypotheses of no age and sex-related effects: At a significance level of $5 \%$ the equal response effects jypothesis cannot te zcjoctes, ieasing us to conclude that Nixon's data do not support the existence of significant differences in Mn levels among occlusal enamel, the inner and outer enamel of the gingival third, and the inner and outer enamel of the gingival third, and the inner and outer enamel of the middle third of the sampled specimens. As for $\mathrm{Hg}$, however, this hypothesis is rejected for a model ignoring sex and age effects (equation (13)). Evident1y, allowing for sexual differences and linear changes in the $\log$ of the Mn concentration with age, though they are not significant terms statistically, removes sufficient variation in the data so that the hypothesis of equal response effects cannot be rejected. More data will be necessary to successfully uncover inequalities in $\mathrm{Mn}$ levels in these types of enamel.

The report by Brudevold and Steadman (1956a) that $\mathrm{Pb}$ tends to accumulate in the outer layers of enamel and decreases rapidly in concentration in successive 
layers of subsurface enamel was challenged in 1972 by Carroll et al (1972) on the basis of an election probe microanalysis of 12 specimens of deciduous teeth from urban children. They noted lead deposition at or in the periphery of hypomineralized zones and in areas adjacent to the pulp, but no lead was detected in surface enamel. Subsequently, however, Shapiro et al (1972) published data involving chemical assay by anodic stripping voltammetry of various portions of known types (molar, canine, etc.) of both permanent and deciduous teeth. The results of analyses of outer and inner enamel from deciduous teeth corroborated in general the earlier findings by Brudevold and Steadman. Shapiro et al (1972) also show that secondary dentine, and in particular, dentine immediately adjacent to the pulp [Shapiro et al (1973)], of both deciduous and permanent teeth contain $\mathrm{Pb}$ at levels approximately 3-9 times those found in bulk enamel and dentine. Malik and Fremlin (1974) have studied lead distribution in teeth by autoradiography of specimens previously activated by charged particle bombardment. Their data support previous observations. in that higher lead levels are found in exterior compared to interior enamel, and in dentine nearer the pulp compared to interior dentine. The differences they record, however, are much smaller than those observed earlier, but their data are too limited for multivariate statistical analysis. Their discovery of a sharp rise in $\mathrm{Pb}$ levels at the $\mathrm{DEJ}$ is noteworthy.

Radium is also known to accumulate in teeth, and Rowland (1963) has shown by autoradiography of teeth of individuals exposed to Ra therapeutically and occupationally, that 'hot spots' develop, particularly in dentine, where a 'hot spot' may contain as much as 100 times the $\mathrm{Ra}$ as the average diffuse level for dentine. In addition, he shows that Ra activity decreases exponentially in successive layers of dentine counted from the circumpulpal dentine, and that the surface layers of enamel contain more radium than Internal enamel. The same is true of cementum vis-a-vis dentine. 
The antimony content of five types of enamel of teeth from Glasgow has been measured by Nixon et al (1967a). As mentioned previously (section 2.4), there is insufficient data for use with a multivariate statistical model that allows for the effects of age and sex. It is clear, however, that such differences as may exist between occlusal enamel, the inner and outer enamel of the gingival third, and the inner and outer enamel of the middle third of the specimens sampled are too small to be of significance.

No new data are available on $\mathrm{Sn}$ and $\mathrm{Sr}$ since the original investigations by Brudevold and Steadman (1956 b) and by Steadman et al (1958) on their distributions in enamel and in enamel and dentine. New data are available on the distribution of $\mathrm{Zn}$ in enamel, however [Nixon et al (1967 b)]. In extending our analysis of this data begun in section 2.4 , to cover the hypothesis of equal response effects, we find that at a significance level of $5 \%$, the data do not support the existence of significant differences in Zn levels in the occlusal enamel, the inner and outer enamel of the gingival third, and the inner and outer enamel of the middle third of the sampled specimens. As in the case of Nixon's Mn data [Nixon et al (1966)], however, this hypothesis is rejected for a model ignoring sex and age effects: age and sex terms, though not significant statistically, evidently remove sufficient variation in the data so that the hypothesis of equal response effects cannot. be rejected. More data will be necessary to uncover inequalities in the $\mathrm{Zn}$ levels in these types of enamel. Taken as a whole, however, these data agree reasonably well with the data published by Brudevold et al (1.963). Finally, Hamilton (1971) has shown that the uranium distribution in individual dental specimens is far from uniform. By neutron irradiation of thin sections of calcified tissue followed by fission track detection (a type 
of autoradiography) he was able to show that the density of tracks associated with uranium was approximately two times greater in dentine than enamel, and five times greater in the pulp than in dentine.

Many studies of the inorganic composition of dental hard tissues involve analyses of bulk enamel and dentine. While not providing the detail available with methods employing activation and/or autoradiography of thin sections of teeth or of methods designed to sample in one way or another discrete layers of enamel, dentine, and root tissue, these studies nevertheless have some bearing on the distribution of elements in individual dental specimens. That the inorganic composition of enamel and dentine are often different is hardly surprising, however, given that the two types of tissue are physiologically distinct.

Retief and co-workers [Retief et al (1970 a, b, 1971 a, b)] have determined the levels of 16 elements ( $\mathrm{Ag}, \mathrm{Al}, \mathrm{Au}, \mathrm{Ba}, \mathrm{Br}, \mathrm{Ca}, \mathrm{Cl}, \mathrm{Co}, \mathrm{Cr}$, $\mathrm{Fe}, \mathrm{Mg}, \mathrm{Mn}, \mathrm{Na}, \mathrm{Sb}, \mathrm{Sr}, \mathrm{Zn}$ ) in enamel and dentine (from the root) by neutron activation analysis involving four procedures for irradiation. The mean concentrations of these elements in enamel and dentine are different: The median factor by which they differ, however, is only 1.47 , the maximum factor being on the order of 9 . If we knew with certainty that the specimens from which the enamel and dentine were obtained were collected independently (i.e., no tooth yielded both enamel and dentine) it would be possible to construct Bonferroni confidence intervals [Miller (1966)] for the differences between enamel and dentine levels of these 16 elements, allowing some measure of significance to be attached to the comparisons (assuming normality, etc.). This is probably not the case, however, and since the required estimates of covariance between enamel and dentine are not available, we are unable to evaluate these comparisons statistically. Söremark, Samsahl, and Lundberg 
[Söremark and Samsah1 (1962a, b); Söremark and Lundberg (1964a; b); Lundberg. et al $(1965 \mathrm{a}, \mathrm{b})]$ have measured the concentrations of 17 elements ( $\mathrm{Ag}, \mathrm{Au}$, $\mathrm{Br}, \mathrm{Ca}, \mathrm{Cl}, \mathrm{Co}, \mathrm{Cr}, \mathrm{Cu}, \mathrm{Fe}, \mathrm{Mn}, \mathrm{Na}, \mathrm{P}, \mathrm{Pt}, \mathrm{Rb}, \mathrm{Sr}, \mathrm{Zn}, \mathrm{W}$ ) in normal human enamel and dentine, and of seven elements ( $\mathrm{Au}, \mathrm{Cd}, \mathrm{Mn}, \mathrm{Na}, \mathrm{Rb}, \mathrm{Sr}, \mathrm{Zn}$ ) in enamel and dentine from unerupted teeth by neutron activation analysis.

For erupted teeth, the median factor by which enamel and dentine differ with respect to the 17 elements studied is 1.39. The maximum factor by which they differ (for $W$ ) is 10.8. For reasons discussed above, we are unable to evaluate these comparisons statistically: However, if dentine and enamel concentrations are assumed independent and normality requirements fulfilled, then $95 \%$ Bonferroni confidence intervals for the differences between mean enamel and dentine levels include zero for all but $\mathrm{Ca}, \mathrm{Fe}, \mathrm{Mn}, \mathrm{Na}, \mathrm{P}$, and $\mathrm{W}$ (for the 16 elements for which mean values were obtained).

Derise (19.73) and Derise et al (1974a, b) have also studied the concentrations of a number of elements in enamel and dentine. From each of 175 permanent caries-free teeth, enamel and dentine samples were assayed for 17 elements (A1, $\mathrm{Ca}, \mathrm{Cl}, \mathrm{Co}, \mathrm{Cu}, \mathrm{F}, \mathrm{Fe}, \mathrm{I}, \mathrm{K}, \mathrm{Mg}, \mathrm{Mn}, \mathrm{Na}, \mathrm{P}, \mathrm{Pb}, \mathrm{Se}, \mathrm{Sr}, \mathrm{Zn}$ ). Differences between enamel and dentine were evaluated statistically by Derise with a three-factor univariate analysis of variance model described in section 2.4 . For each of the 17 elements, the differences between enamel and dentine levels were found to be significant (some two-factor interactions between the variables, age and sex, and tissue type, and one three-factor interaction were also significant, however). Based on over-all arithmetic mean concentrations, the median factor by which enamel and dentine differ is 1.39 , the maximum being 4.67 for $\mathrm{Cl}$. While significant, these differences are not overwhelming and one is left to speculate about the possible effect of nonindependence of enamel and dentine concentrations on the underlying statistical analysis. The significance of the differences between enamel and dentine would probably degrade under multivariate analysis of variance. 
Steinnes et al (1974) describe analysis of $\mathrm{La}, \mathrm{Sm}, \mathrm{Tb}$, and $\mathrm{Yb}$ in a limited number of enamel and dentine samples. The data are too few for statistical analysis, but for $\mathrm{La}$ and $\mathrm{Sm}$, enamel and dentine levels are within an order of magnitude of one another. $\mathrm{Yb}$ and $\mathrm{Tb}$ were not detected in any of the samples. Hadjimarkos and Bonhorst (1962) report on the $F$ and Se contents of enamel and dentine. Again, these two tissues contained comparable levels of the named elements.

There are a substantial number of investigations in which enamel and dentine have been assayed for a single element. These include Cd [Iwakura (1972)], F [McClure and Likins (1951); Jackson and Weidmann (1959); Shaw et al (1959)], $\mathrm{K}$ [Steadman et al (1941)], Mn [Battistone et al (1967)], Pb [Pfrieme (1934); Maulbetach and Rutishauser (1936); Ryt 8 maa and Tuompo (1974); Shapiro et al (1973)], S [Hachino (1969a), Se [Hadjimarkos and Bonhorst (1959)]. Sn [Pearlman et al (1970)], and Zn [Cruickshank (1949); Babicky and Taylor (1966)]. With the exception of Rytbmaa and Tuompo's observation that lead is found in enamel at levels 98-177 times those found in dentine, an observation at variance with most other work on lead, all of these studies tend to show that differences between bulk enamel and dentine, though real, are limited. Ratios tend to be in the range $0.1=10$.

In order to round out our statistical analysis begun in section 2.4 , we have tested the hypothesis of equal response effects (equal Mn levels in enamel and dentine) for Battistone et al's (1967) data on Mn levels in the enamel and dentine of erupted and unerupted teeth. At a significance level of $5 \%$, this hypothesis is rejected for erupted teeth but not for unerupted teeth. Hence, Battistone's data do support the existence of significant differences in the Mn levels of enamel and dentine in erupted teeth. The difference (recall that concentrations were logarithmically transformed for this analysis) amounts to a 
ratio of 7.6 if evaluated in terms of the parameters of the multivariate model (equation (16)).

In general, it is our impression that the inorganic compositions of bulk enamel and dentine are similar. This is encouraging insofar as large-scale multielement analyses are concerned where sample preparation time is an important factor. Thin sections of enamel and/or dentine, on the other hand, may show considerably greater fluctuation in composition permitting selection of tissue which will maximize one's ability to make meaningful chemical analyses. 


\section{$\underline{2.7}$ Tooth Type/Intermouth Variation}

The extent to which type of tooth is an important factor in determining its inorganic composition has received limited attention in the literature we have surveyed. A related problem, comparison of the variability in the composition of teeth from the same mouth to teeth from different mouths, has received less attention. Both of these factors are of potential concern in comparisons of data from different sources or where data from the same source are being analyzed with respect to external variables such as age, sex, or sample provenance. Unfortunately, most of the available data do not permit simultaneous consideration of the many variables that might affect the composition of teeth (with complex analysis of variance models, for example). The needed data are often unavailable; elsewhere, the data are insufficient or otherwise poorly structured. Separate evaluation of the significance of each variable (by one-way analysis of variance, for example) is risky and may fail to reveal significant relationships in the data if more than one factor is actually significant.

There is very little evidence suggesting that type of tooth is a significant factor for the inorganic composition of teeth. The failure of Crowell et al (1934) to find differences between types of (whole) teeth with respect to their $\mathrm{Ca}, \mathrm{Mg}$, and $\mathrm{P}$ content has already been mentioned: Nor do Söremark and Samsahl (1962a) find any significant variation in the $\mathrm{Au}, \mathrm{Br}, \mathrm{Ca}$, $\mathrm{Cl}, \mathrm{Cu}, \mathrm{Mn}, \mathrm{Na}, \mathrm{P}, \mathrm{Sr}, \mathrm{W}$, and $\mathrm{Zn}$ content of enamel from upper and lower first bicuspids, molars, and canine teeth, though details are not published. The concentrations of these elements in the dentine of upper and lower first bicuspids have also been found to be similar [Söremark and Samsahl (1962b)]. The $\mathrm{Ag}, \mathrm{Co}, \mathrm{Cr}, \mathrm{Fe}, \mathrm{Rb}$, and $\mathrm{Pt}$ levels in both énamel and dentine [Söremark and Lundberg (1964 a, b)] of upper and lower first bicuspids are similar as well. 
More comprehensive results have been compiled by Curzon et al (1975) in a study of matched pairs of upper left and right first bicusplds from 18 11-year old individuals of Dunedin, New Zealand. The enamel from these specimens failed to reveal significant differences. between left and right teeth: The elements for which this inference held were $\mathrm{Ag}, \mathrm{Al}, \mathrm{B}, \mathrm{Ba}, \mathrm{Br}, \mathrm{Cd}$, $\mathrm{Cu}, \mathrm{F}, \mathrm{Fe}, \mathrm{Li}, \mathrm{Mn}, \mathrm{Mo}, \mathrm{Nb}, \mathrm{Ni}, \mathrm{Pb}, \mathrm{Rb}, \mathrm{S}, \mathrm{Sb}, \mathrm{Se}, \mathrm{Sn}, \mathrm{Sr}, \mathrm{Ti}, \mathrm{V}, \mathrm{Zn}$, and $\mathrm{Zr}$, and represent the 25 elements (out of 28) for which sufficient data were available. (The univariate statistical that applied by Curzon and coworkers, a 'test of paired differences,' is equivalent to a two-way analysis of variance model with no interaction.) Data on the $\mathrm{Ca}, \mathrm{Cd}, \mathrm{Cu}, \mathrm{Pb}$, and Zn content of whole teeth have been tabulated by Kaneko (1972) for nine individuals from whom more than one tooth of known type was obtained. Because of its limited nature, however, our analysis of this data will be deferred until inter-and intra-mouth variation is discussed. In a study of the C, Ca, and P content of sections of teeth, Stubbins and Fremlin (1967) find that the carbon content of inner enamel from molars is significantly greater than that of premolars; further work would be desirable to substantiate this difference, which is small.

Among single element studies, Lockeretz (1975) has found significant differences in the $\mathrm{Pb}$ content of deciduous incisors, cuspids, and molars. These data are extensive. A partial explanation of these differences may have been provided by Strehlow (1972) in his study of the $\mathrm{Pb}$ content of the teeth of primates that have ingested ${ }^{210} \mathrm{~Pb}$. He found that since $\mathrm{Pb}$ was preferentially deposited in the root, the ratio of the bulk of the crown to the root was an important variable in explaining differences between the $\mathrm{Pb}$ contents of different types of (whole) teeth. Exfoliation of deciduous 
teeth, however, is accompanied by root resorption with loss of a significant fraction of total lead.

Variation in the composition of teeth as a function of tooth type has also been reported by Belekhova (1966) for $\mathrm{V}$ (cuspids were approximately 1.7 times higher in $\mathrm{V}$ than molars) and by Lazarev (1962) for $\mathrm{Zn}$ (the variation reported is slight). The remaining data available shows little relationship between tooth type and inorganic composition. These include data on Cu [Nezhivenko (1961a)], Se[Mamedova (1965)], ${ }^{90} \mathrm{Sr}[$ Aarkrog (1971)], and Zn [Cruickshank (1949)]. We also mention here, data that have been compiled for some elements as a function of tooth type but that are too limited in one or more respects for analysis. These include data on As [Götte and Hattemer (1955); Sairenji et al (1961)], Hg [Nixon et al (1965)], Pb [Shapiro et al (1972, 1973)], Mn [Nixon et al (1966)], Sb [Nixon et al (1967a)], U [Ikeda et al (1967)], and $\mathrm{Zn}$ [Nixon et al (1967b)]. Calonius and Visapää (1965) have published semi-quantitative data of a similar type for X-ray fluorescence analysis of teeth for $\mathrm{Ag}, \mathrm{Br}, \mathrm{Ca}, \mathrm{Cl}, \mathrm{Co}, \mathrm{Cr}, \mathrm{Cu}, \mathrm{Fe}, \mathrm{K}, \mathrm{Mn}, \mathrm{Ni}, \mathrm{P}, \mathrm{Pb}, \mathrm{Pd}$, $\mathrm{Sb}, \mathrm{Sn}, \mathrm{Sr}, \mathrm{Zn}$, and $\mathrm{Zr}$.

Intra- versus inter-mouth variation has been specifically examined by Crowell et al (1934) with respect to the Ca, Mg, and P content of teeth; they have noted that for their sample, variation in the composition of teeth from the same mouth was comparable to variation observed between mouths. Our own analysis of the $\mathrm{Cd}, \mathrm{Cu}, \mathrm{Pb}$, and $\mathrm{Zn}$ data tabulated for individuals by Kaneko (1972) reveals that between-mouth variation exceeds within-mouth variation by a significant amount for $\mathrm{Pb}$ only. This is disconcerting in view of the fact that one might have hoped to employ the trace element content of these teeth to classify their donors by residence. We caution, however, that these data are 
of limited scope, that our analysis is in terms of univariate one-way analysis of variance models, and that censored data (extensive for $\mathrm{Cd}$ ) have been treated as missing data.

Battistone et al (1967) also use one-way analysis of variance to infer. significant inter-mouth variation (relative to intra-mouth variation) of the Mn content of both enamel and dentine. Nixon et al (1966) have noted similar consistency of Mn levels of enamel for teeth derived from the same mouth relative to teeth from different mouths.

We have observed previously that where more than one element or tissue type is surveyed [e.g., by Curzon et al (1975); Kaneko (1972); Crowell et al (1934); and by Nixon et al (1966); Battistone et al (1967)], multivariate statistical techniques and techniques for simultaneous inference may yield more meaningful analyses. However, careful consideration must be given to responses that are not independent. For example, Curzon's data are multivariate: Two teeth are obtained from each mouth and the enamel of each tooth is characterized with respect to 25 elements. Not only should allowance be made for non-independence among the 25 elements, but also for possible non-independence in levels obtained for teeth from the same mouth. The latter is allowed for in Curzon's 'paired t-test', but not the former. However, with some simplifying assumptions, simultaneous confidence intervals can be constructed for comparison of left and right teeth from the paired t-test. Since these intervals will be broader than those implied by Curzon's tabulation, and since all his intervals already include zero, the inferred equality between concentrations in left and right teeth goes unchallenged. "Battistone's data are also multivariate: Two teeth are obtained from each of 12 individuals and the enamel and dentine of each tooth characterized with respect to its Mn concentration. Not only are $\mathrm{Mn}$ levels in enamel and dentine within a tooth probably not independent; Mn levels in tissue obtained 
from different teeth from the same mouth are probably also not independent. These types of non-independence are not allowed for in a univariate one-way analysis of variance.

Considerably more effort has been expended in investigating differences in the inorganic composition of different types of teeth if, in distinguishing between types of teeth, one includes as a criterion the distinction between erupted and unerupted teeth. The available literature includes a semiquantitative multielement survey by Grippaudo (1963) (Al, Ca, Co, Cr, Cu, Fe, $\mathrm{K}, \mathrm{Mg}, \mathrm{Mn}, \mathrm{Na}, \mathrm{Ni}, \mathrm{P}, \mathrm{Pb}, \mathrm{Si}, \mathrm{Sn}, \mathrm{Sr}, \mathrm{Ti}, \mathrm{V}, \mathrm{Zn}$ ), quantitative multielement surveys by Lundberg et al (1965 a,b) (Au, Cd, Mn, Na, Rb, Sr, Zn), Steinnes et al (1974) ( $\mathrm{La}, \mathrm{Sm}, \mathrm{Tb}, \mathrm{Yb})$, Langmyhr et al (1974) (Cd, $\mathrm{Pb})$, and by Langmyhr et al (1975) ( $\mathrm{Ag}, \mathrm{Mn}, \mathrm{Zn})$, and quantitative single element surveys Involving $\mathrm{Cu}$ [Brudevold and Steadman (1955)], F [Brudevold et al (1956c)], $\mathrm{Hg}$ [Nixon et al (1965)], Mn [Battistone et al (1967); Shimada (1971)], Pb [Brudevold and Steadman (1956a)], Sn [Brudevold and Steadman (1956 b)], Sr [Steadman et al (1958); Gedalia et al (1973)], and Zn [Brudevold et al (1963)]. Where these data have been specifically analyzed in an effort to uncover differences in composition between erupted and unerupted teeth, the conclusions vary with the element. For example, Lundberg et al (1965a) find that the enamel of unerupted bicuspids is (significantly) higher in Mn and $\mathrm{Rb}$ than enamel from erupted bicuspids, while $\mathrm{Au}$, detectable in the latter, was below detection limits in the former. Lundberg et al (1965b) find a similar pattern of differences in the composition of dentine from unerupted and erupted bicuspids, though only $\mathrm{Rb}$ is considered to be present at significantly different levels. Brudevold and Steadman (1955), though finding that the $\mathrm{Cu}$ content of enamel from erupted teeth was higher than for unerupted teeth (20 vs $7 \mathrm{ppm}$ ), did not consider the differences significant. Nixon et al (1965), however, conclude that at least for $\mathrm{Hg}$ such differences are 
significant and attributed them to exposure of enamel to an oral environment contaminated with Hg from amalgam fillings. The $\mathrm{F}$ content of the enamel of a sample of unerupted teeth was considerably below values obtained for unerupted teeth, leading Brudevold et al (1956c) to conclude that posteruptive uptake of $\mathrm{F}$, at least by the outer layers of enamel, is significant. Battistone et al (1967) have found that the Mn concentration in dentine of the erupted teeth of female patients is significantly below the value obtained from unerupted teeth. The differences were even more significant for older females (both for enamel and dentine) which lead Battistone and his colleagues to suggest that there is a gradual posteruptive decrease in the Mn content of female teeth that is not found in male teeth. These conclusions may require modification in view of the results we have obtained with multivariate statistical models (e.g., in sections 2.4 and 2.5). $\mathrm{Pb}$ has been found to be present. in enamel from erupted teeth at levels 2-3 times those found for unerupted teeth by Brudevold and Steadman (1956a). A similar pattern was found for Sn by Brudevold and Steadman (1956b), but the effect was at least partially attributed to contamination by amalgam fillings that were present in many of the erupted teeth prior to analysis. No differences were observed when the analysis of erupted teeth was restricted to intact samples. Sr is evidently present at comparable levels in the enamel of erupted and unerupted teeth [Steadman et al (1958)], a result that has been confirmed in a study by Gedalia et al (1973) on the enamel of (pairs) of erupted and unerupted third molars from the same mouth for a series of individuals. (The correlation between erupted and unerupted enamel was found to be highly significant.) Finally, Brudevold et al (1963) have found that $\mathrm{Zn}$ is present in the outer layers of enamel from erupted teeth in somewhat greater concentration than is found in unerupted teeth of the same provenance, the difference being greatest for the outermost layer. 
The remaining data on the inorganic composition of erupted and unerupted teeth is incidental. Steinnes et al (1974), for example, tabulate some data on the $\mathrm{La}, \mathrm{Sm}, \mathrm{Tb}$, and $\mathrm{Yb}$ concentrations of enamel and dentine from a few teeth, one of which was unerupted. Langmyhr et al (1974) tabulate data for $\mathrm{Cd}$ and $\mathrm{Pb}$ in 14 whole teeth, of which six are unerupted. The same sample of teeth, evidently employed later [Langmyhr et al (1975)], has also been assayed for $\mathrm{Ag}, \mathrm{Mn}$, and $\mathrm{Zn}$. In using these data in a one-way multivariate analysis of variance (employing log-transformed data), we have ignored the fact that some of the values tabulated are means of several replicate analyses: These data do not, in their present form, support the existence of significant differences in the $\mathrm{Ag}, \mathrm{Cd}, \mathrm{Mn}, \mathrm{Pb}$, and $\mathrm{Zn}$. concentrations of erupted and unerupted teeth.

$v \cdot v$ 


\section{$\underline{\underline{2.8}} \underline{\underline{\text { Miscellaneous }}}$}

The literature on the inorganic composition of dental hard tissues includes numerous references devoted to the effect on composition of factors other than those surveyed above. These include the condition of the teeth (carious and precarious vs noncarious tissue), the presence or absence of periodantal disease, the occupation of the donor, the health of the donor (the presence or absence of specific diseases), the effects of medical therapy (As-devitalization of pulp tissue and treatment of bilharzia with $\mathrm{Sb}$, for example); and the effects of corrective dental therapy (the presence or absence of restorations, orthodontic devices, etc.).

Data relating to the fnorganic composition of carious tissue have been compiled by Crowell et al (1934) (Ca, Mg, P), Hoogland (1948) (F, Mo), Pieruccini and Zotti (1949) (Mn, Sr), Grippaudo (1962) (A1, Ca, Co, Cr, Cu, $\mathrm{Fe}, \mathrm{K}, \mathrm{Mg}, \mathrm{Mn}, \mathrm{Na}, \mathrm{Ni}, \mathrm{P}, \mathrm{Pb}, \mathrm{Si}, \mathrm{Sn}, \mathrm{Sr}, \mathrm{Ti}, \mathrm{V}, \mathrm{Zn}), \mathrm{Vikhm}$ (1962) and Vihm (1964) (Ag, N1), Storozheva (1963) (Pb, Sn), Johansen (1965) (Al, Ca, F, Fe, $\mathrm{Mg}, \mathrm{Mn}, \mathrm{P}, \mathrm{Pb}, \mathrm{Sn}, \mathrm{Sr}$ ), and by Little and Steadman (1966) (Ag, $\mathrm{Al}, \mathrm{Cr}, \mathrm{Cu}, \mathrm{F}$, $\mathrm{Fe}, \mathrm{Mn}, \mathrm{Pb}, \mathrm{Si}, \mathrm{Sn}, \mathrm{Sr}, \mathrm{Zn}$ ), among others. Additionai data on single elements is available, comprising As [Vikhm (1963)], Cu [Ginzburg and El'piner (1947); Nezhivenko (1961b)], F [Levine (1972)], Mg [Kaushansky (1932); Halsworth et al (1972)], and Mn [Lazarev (1962)]. In general the destruction of enamel and dentine by the carious process leads to changes in the composition of these tissues; however, the significance of these changes, especially with respect to trace constituents other than $F$, has not been evaluated, nor is it known to what extent 'accidents' of sampling contribute to these results. For example, since weight-based concentrations must add up to $100 \%$ of the sample weight, loss of a substantial fraction of a major element (or mineral) such as $\mathrm{Ca}$, will lead to changes (increases) in the concentrations of all the trace constituents though they may in no way be involved in the chemistry of the carious process. 
Periodontal disease and its effect on the inorganic composition of teeth has been the subject of some study, especially in the Soviet literature. Data on pyorrhea have been compiled by Vihkm (1962) and Vihm (1964) (Ag, Ni), and by Nezhivenko et al (1967) ( $\mathrm{Ag}, \mathrm{As}, \mathrm{Cu}, \mathrm{Mn}, \mathrm{Ni}, \mathrm{Pb}, \mathrm{Sb}, \mathrm{Se}, \mathrm{Ti}$ ). Additional data on single elements have also been compiled, including data on As [Vikhm (1963)], Cu [Nezhivenko (1961a)], Mg [Kaushansky (1932)], Mn [Serebryakova (1963); Shimada (1971)], ${ }^{90}$ Sir [Marei et al (1972)], and Zn [Cruickshank (1937)]. Though changes in composition have been noted, their slgniflcance remains a matter of conjecture and more systematic and carefully planned studies will be necessary for their evaluation.

The health of the individual from whom the specimens for analysis have been obtained may also influence the inorganic composition of teeth. Specific examples may be found in Nezhivenko et al (1967) ( $\mathrm{Ag}, \mathrm{As}, \mathrm{Cu}, \mathrm{Mn}, \mathrm{Ni}, \mathrm{Pb}, \mathrm{Sb}$, Se, Ti ). Single elements for which data are available include Cu [Nezhivenko (1961)] and Zn [Lazarev (1962)].

The relationship between occupation and the composition of teeth may be viewed as one primarily between an individual's micro-environment and the elemental composition of his teeth. Mamedov and Anifaev (1974), for example, have found that of the elements they surveyed ( $\mathrm{Ag}, \mathrm{Al}, \mathrm{Cu}, \mathrm{Fe}, \mathrm{La}, \mathrm{Mg}, \mathrm{Mn}$, Mo, Na, Si, Ti, V, $\mathrm{Zr}$ ), Al, Fe, La, and Si were found in greater-than-normal amounts in samples of teeth obtained from workers of iron-ore enriching plants. Elevated $\mathrm{Pb}$ levels have been found in the teeth of metal workers by Wyss (1951), and (evidentiy) much higher than normal levels of $\mathrm{Ra}$ in the teeth of (Ra-poisoned) 'dial' painters by Rowland (1963).

The relationship between medical therapy and the composition of teeth is of a similar nature. For example, teeth that have been subject to Asdevitalization show, even after many years have passed, elevated As levels 
[Sairenji et a1 (1961); Götte and Hattemer (1955)], and Wainwright et a1 (1953) has found that the ${ }^{131} \mathrm{I}$ that localizes in teeth following administration for thyroid therapy, remains there for at least two weeks (the period of the study). Ra, possibly acquired therapeutically, has also been detected in a tooth obtained for analysis many years after Ra administration [Rowland (1963)]. $\mathrm{Sb}$, however, though found at somewhat elevated levels in the teeth of Egyptian patients treated with antimony for bilharzia, is not significantly above levels found in enamel from untreated Egyptian patients [Nixon et al (19697a)].

The presence of restorations or prosthetic dental devices in the mouth of an individual from whom a specimen is obtained for chemical analysis can have a profound effect on its composition, especially with respect to its trace element content. Several examples have already been cited above in other contexts. Fields et al (1950) was among the first to provide evidence on the migration of restoration metals into neighboring tissue. The most thorough and recent study, however, has been published by Söremark et al (1968) and involves the Ag, Au, $\mathrm{Co}, \mathrm{Cr}, \mathrm{Cu}, \mathrm{Hg}$, and $\mathrm{Zn}$ content of enamel and dentine of teeth restored with various types of material or in contact with chromium-cobalt clasps. Of particular interest is the fact that many of these trace elements are present throughout restored teeth at higher-than-normal levels (depending on the type of filling) and that the effect may amount to many orders of magnitude for tissue obtained from points close to (though not in direct contact with) the fillings. The effect of chromium-cobalt clasps, is equally pronounced for tissue (enamel) in contact with them and, surprisingly, for dentine, too. 


\subsection{Summary}

In the preceding chapter, evidence concerning factors thought to influence the inorganic composition of teeth was reviewed and evaluated. Summarized below, by topic, are the results of this evaluation.

Geographic vaxiation: There exists convincing evidence that, at least for some elements, the inorganic composition of teeth is related to the donor's geographical residence. This evidence is particularly strong for $\mathrm{F}, \mathrm{Pb}, \mathrm{Se}$, and $\mathrm{Sr}$, and less so for a host of other elements, including $\mathrm{Ag}, \mathrm{B}, \mathrm{Br}, \mathrm{Ca}, \mathrm{Cd}, \mathrm{Cu}, \mathrm{Fe}, \mathrm{Mn}, \mathrm{Mo}, \mathrm{Nb}$, Ra, S, U, Zn, and Zr. Except for Cd, F, Ra, Se, Sr, and U, however, the relationship between environmental concentration and dental composition has not been investigated.

Age: The relationship between age and dental composition is well established for $\mathrm{F}, \mathrm{Pb}$, and ${ }^{90} \mathrm{Sr}$. Variation in dental composition with age, though possibly significant for other elements, has been difficult to evaluate, because the published data are frequently poorly structured (it is not clear, for example, that observed variation can be attributed to age or whether it is due to some other uncontrolled, possibly unknown variable).

Sex: Published analyses notwithstanding, we do not believe the data support the existence of significant sex-related differences in the inorganic composition of dental hard tissues.

Distribution: The distribution of trace elements within dental specimens is far from uniform; this is particularly evident with respect to the depth 
within a tooth from which a sample, dentine or enamel, is obtained. Frequently concentrations are highest in the external layers of enamel and in the circumpulpal dentine. Bulk enamel. and dentine also differ in composition though less markedly. Differences larger than an order of magnitude are unusual; more commonly, concentrations in these two tissues differ by about a factor of two. Tooth type/Intermouth variation: Deciduous incisors, molars, and canines are convincingly different with respect to $\mathrm{Pb}$ concentration. The bulk of evidence on the composition of teeth, however, reveals little correlation between tooth type and composition, except where erupted and unerupted specimens are compared. The differences that occur here depend on the particular element considered, however.

Inter- and intra-mouth variation of the composition of teeth has received limited attention. Only for $\mathrm{Mn}$, has inter-mouth variability been observed to exceed intra-mouth variability by a significant amount, though one might hope that future effort in this area will reveal'this phenomenon to be much more widespread. Indeed, this must be true if any meaningful conclusions are to be drawn from data on the inorganic composition of teeth.

Miscellaneous: The effect on dental composition of a variety of miscellaneous factors has also been considered and the reader is referred to the text of this report for their review. Generally, the data have not lent themselves to critical evaluation. 


\section{THIS PAGE}

\section{WAS INTENTIONALLY LEFT BLANK}


17. Brudevold, F. and Steadman, L. T. (1956a). The distribution of lead in human enamel. J. Dent. Res. 35, 430-437.

18. Brudevold, F. and Steadman, L. T. (1956b). A study of tin in enamel. J. Dent. Res. 35, 749-752.

19. Brudevold, F., Gardner, D. F., and Smith, F. A. (1956c). The distribution of fluoride in human enamel. J. Dent. Res. 35, 420-429.

20. Brudevold, F., Steadman, L. T., and Smith, F. A. (1960). Inorganic and organic components of tooth structure. Ann. New York Acad. Sci. 85, 110-132.

21. Brudevold, F., Steadman, L. T., Spinelli, B. H., Amdur, B. H., and Gron, P. (1963). A study of zinc in human teeth. Arch. Oral Biol.: 8 , 135-144.

22. Brudevold, F. and söremark, R. (1967). Chemistry of the mineral phase of enamel. Structural and chemical organization of teeth, A. E. W. Miles, Ed., Academic Press, New York, Vol. 2, 247-277.

23. Bryant, F. J., Henderson, E. H., and Holgate, w. (1960). Strontium 90 in human teeth. Brit. Dental J. 108, 291-294.

24. Burnett, G. W. and Zenewitz, J. A. (1958). Studies of the composition of teeth. VII. The moisture content of calcified tissues. J. Dent. Res. 37, 581-589.

25. Burrell, D. C. (1972). Flame spectrophotometric trace analysis. Ultrapurity, M: Zief and R. Speights, Eds., Marcel Dekker, Inc., N. Y., 477-534.

26. Butler, F. E. (1961). Strontium-90 in human teeth. Nature 189, 848-849.

27. Calonius, P. E. B. and Visapaa, A. (1965). The inorganic constituents of human teeth and bone examined by $\mathrm{x}$-ray emission spectrography. Arch. Oral Biol. 10, 9-13.

28. Campbell, A. M. G., Herdan, G., Tatlow, W. F. T., and Whittle, E. G. (1950). Lead in relation to disseminated sclerosis. Brain 73, 52-71.

29. Carach, J., Csupka, S., Petrasova, M., Cechvala L., and Sauberer S. (1970). Accumulation of strontium-90 in deciduous and permanent teeth in slovakia. Bratislav. Lek. Listy $53,257-265$.

30. Carroll, K. G., Needleman, H., Tuncay, O. C., and Shapiro, I. M. (1972). The distribution of lead in human deciduous teeth. Experientia 28, 434-435.

31. Cohen, Jr., A. C. (1959). Simplified estimators for the normal distribution when samples are singly censored or truncated. Technometrics 1, 217-237.

32. Crowell, Jr., C. D., Hodge, H. C., and Line, W. R. (1934). Chemical analysis of tooth samples composed of enamel, dentine, and cementum. J. Dent. Res. 14, 251-268. 
33. Cruickshank, D. B. (1936). The natural occurrence of zinc in teeth. Brit. Dental J. 61, 530-531.

34. Cruickshank, D. B. (1937). The natural occurrence of zinc in teeth. Brit. Dental J. 63, 395-399.

35. Cruickshank, D. B. (1949). The frequency distribution of the zinc concentrations in the dental tissues of the normal population. Biochem. J. 44, 299-302.

36. Curzon, M. E. J., Kubota, J., and Bibby, B. G. (1971). Environmental effects of molybdenum on caries. J. Dent. Res. 50, 74-77.

37. Curzon, M. E. J., Losee, F. L., Brown, R., and Taylor, H. E. (1974). Vanadium in whole human enamel and its relationship to dental caries. Arch. Oral Biol. 19 , 1161-1165.

38. Curzon, M. E. J., Losee, F. L:, and Macalister, A. D. (1975). Trace elements in the enamel of teeth from New Zealand and the U. S. A. N. Z. Dental J. 71, 80-83.

39. Degenhardt, H. (1957). Geochemical distribution of zirconium in the lithosphere. Geochim. et Cosmochim. Acta 11, 279-309.

40. Derise, N. L. (1973). The mineral composition of normal human enamel ana dentine and the relation of composition to dental caries. Ph.D. Thesis, Virginia Polytechnic Institute and State University.

41. Derise, N. L., Ritchey, S. J., and Furr, A. K. (1974a). Mineral composition of normal human enamel and dentin and the relation of composition to dental caries. I. Macrominerals and comparison of methods of analyses. J. Dent. Res. 53, $847-852$.

42. Derise, N. L. and Ritchey, S. J. (1974b). Mineral composition of normal human enamel and dentin and the relation of composition to dental caries. II. Microminerals. J. Dent. Res. 53, 853-858.

43. Desgrez, A. and Meunier, J. (1927). Presence of lithium and strontium in human teeth and bones and their chemical state of combination. Compt. Rend. $185,160-163$.

44. de la Burde, B. and Shapiro, I. M. (1975). Dental lead, blood lead, and pica in urban children. Arch. Environmen. Health 30, 281-284.

45. Dmitriev, I. B. (1967). Spectral analysis of the teeth. An aid in medicolegal examination. Sudebno-Med. Ekspertiza, Min. Zdraookhr. SSSR 10, 32-35.

46. Dmitriev, I. B. (1969). Use of chemical composition of tooth enamel in forensic medicine. SB. Tr. Sud. Med. Sud. Khim, 393-397.

47. Dolinsek, F., Stupar, J., and Spemko, M. (1975). Determination of aluminium in dental enamel by the carbon cup atomic-absorption method. Analyst 100, 884-890.

48. Drea, W. F. (1936). Spectrum analysis of dental tissues for trace elements. J. Dent. Res. 15, 403-406. 
49. Dulka, J. J. and Risby, T. H. (1976). Ultratrace metals in some environmental and biological systems. Anal. Chem. 48, 640A-653A.

50. Fields, L. B. and Charles, G. W. (1950). A spectrographic investigation of trace elements in human teeth. Proc. Okla. Academy of Science 31, 47-48.

51. Fin'ko, V. I. (1964). Distribution of rare and rare earth elements and minerals in effusive rocks. Izv. Akad. Nauk. SSSR, Ser. Geol. 29, 90-94.

52. Flanagan, F. J. (1976). An approximate method of computing errors in trace analysis due to sampling heterogeneous solids. Accuracy in trace analysis, sampling, sample handling, analysis. Proc. of the 7 th Materials Research Symposium, NBS Special Publication 422.

53. Gassmann, T. Chemical investigation of teeth. Z. Physiol. Chem. 55, 455-465.

54. Gassmann, T. Chemical investigations of teeth. Z. Physiol. Chem. 63, 397-400.

55. Gassmann, T. (1916). Detection of selenium in bones and teeth. z. Physiol. Chem. 97, 307-310.

56. Gassmann, T. (1917). Estimation of selenium in bones, teeth and urine. z. Physiol. Chem. 100, 182-189.

57. Gedalia, I., Yariv, S., Nayot, H., and Eidelman, E. (1973). The strontium concentration in developing mandibles and teeth in enamel of non-erupted and erupted teeth of human beings. Trace Element Metabolism in Animals- 2 . University of Wisconsin, 1973, 461-464.

58. Ginzburg, M. M. and Elpiner, I. E. (1947). Polarographic determination of copper and zinc in healthy and carious teeth. Stomatologiya 13-16.

59. Glass, R. L. (1967). Radiographic evidence of tin uptake by human tooth structure. Arch. Oral Biol. 12, 401-406.

60. Götte, H. and Hattemer, J. A. (1955). Radioaktivierungs-analytischer nachweis von arsen in zahnen. 2 . Naturforsch. 10B, 343-345.

61. Grippaudo, G. (1963). I microelementi dei tessuti duri dei denti esaminati con il metodo della spettrografia ottica. Ann. Stom. 185-192.

62. Habercam, J. W., Keil, J. E., Reigart, J. R., and Croft, H. W. (1974). Lead content of human blood, hair, and deciduous teeth. Correlation with environmental factors and growth. J. Dent. Res. 5 , 1160-1163.

63. Hachino, H. (1969a). Sulfur content of tooth. II. Sulfur content of human enamel and dentine. Hoi, Kanshiki Narabini Shakai Igaku Zasshi 6, 99-106.

64. Hachino, H. (1969b). Sulfur content of tooth. II. Sulfur content of transparent dentine. Hoi, Kanshiki Narabini Shakai Igaku Zasshi 6. 126-132. 
65. Hadjimarkos, D. M. (1956). Geographic variations of dental caries in Oregon, Part VII. J. Pediatr. 48, 195-201.

66. Hadjimarkos, D. M., and Bonhorst, C. W. (1959). The selenium content of human teeth. Oral Surg., Oral Med., and Oral Pathol. 25, 113-116.

67. Hadjimarkos, D. M., and Bonhorst, C. W. (1962). Fluoride- and seleniumlevels in contemporary and ancient Greek teeth in relation to dental caries. Nature 193, 177-178.

68. Hadjimarkos, D. M. (1969). Selenium. A caries-enhancing trace element. Caries Res. 3, 14-22.

69. Hadjimarkos, D. M. (1971). The role of selenium in dental health. Trace substances in environmental health - IV., D. D. Hemphill, Ed., University of Missouri, Columbia, Missouri, 301-306.

70. Hadjimarkos, D. M. (1973). Selenium in relation to dental caries. Fd. Cosmet. Toxicol. 11, 1083-1095.

71. Hakamori, S. and Tamura S: (1951). The presence of bismuth in fish bones and human teeth as revealed by the cathodo-luminescence method of analysis. Bull. Chem. Soc. Japan 24, 117.

72. Hakamori, S., Umerayashi, H., and Tamura, S. (1958). Analytical chemistry of trace elements and mineral constituents in biological materials. VIII. Cathodo-luminograms of human teeth. Sci. Repts. Tohuku Univ. 42, 77-84.

73. Hallsworth, A. S., Robinson, C., and Weatherel1, J. A. (1972). Mineral and magnesium distribution within the approximal carious lesion of dental enamel. Caries Res. 6 , 156-168.

74. Hamilton, E. I. (1971). The concentration and distribution of uranium in human skeletal tissues. Calc. Tiss. Res. 7, 150-162.

75. Hardwick, J. L. and Martin, C. J. (1967). A pilot study using mass spectrometry for the estimation of the trace element content of dental tissues. Helv. Odont. Acta 11, 62-70.

76. Harris, W. E. and Kratochvil, B. (1974). Sampling variance in analysis for trace components. Preparation of reference samples. Anal. Chem. 46, 313-315.

77. Hartley, H. O. and Hocking, R. R. (1971). The analysis of incomplete data. Biometrics 27, 783-823.

78. Harville, D. A. (1977). Maximum liklihood approaches to variance component estimation and to related problems. J. Amer. Statist. Assoc. 72, 320-338.

79. Healy, W. B. (1968). Barium content of teeth, bone and kidney of twin sheep raised on pastures of differing barium content. Arch. Oral Biol. 13, 559-563.

80. Helsby, C. A. (1974). Determination of strontium in human tooth enamel by atomic absorption spectrometry. Anal. Chim. Acta 69, 259-265. 
81. Higashi, T. and Kawai J. (1969). Measurement of micro elements in dental pulp by activation analysis. Kokobyo-Gakkai Zasshi 18, 357-361.

82. Hodge, H. C., Gavett, E., and Thomas, I. (1946). The adsorption of strontium at forty degrees by enamel, dentine, bone, and hydroxyapatite as shown by radioactive isotope. J. Biol. Chem. $163,1-6$.

83. Hoogland, P. L. (1949). Trace elements and dental caries. Mededel. Lab. Physiol. Chem. Univ. Amsterdam en Nederlands Inst. Volksvoed. 11.

84. Husler, J. W. and Cruft, E. E. (1969). Atomic absorption determination in calcium sulfate minerals using organic enrichment technique. Anal. Chem. 41, $1688-1690$.

85. Ikeda, N., Kimura, K., Hasebe, N., Suzuki, M., Sasaki, E., and Abe, T. (1967). Radioactivation method for the determination of uranium in human teeth. Radioisotopes 16, 247-250.

86. Inami, E. (1969a). Sulfur content of dental calculus. I. Sulfur content of supragingival calculus. Hoi, Kanshiki Narabini Shakai Igaku Zasshi 6, 133-139.

87. Inami, E. (1969b). Sulfur content of dental calculus. II. Sulfur content of subgingival calculus. Hoi, Kanshiki Narabini Shakai Igaku Zasshi 6 , 16i-167.

88. Iwakura, M. (1972). Quantitative analysis of cadmium in the teeth of the inhabitant in a cadmium contaminated region. Koku Eisei Gakki Zasshi 22, 1-9.

89. Jackson, D. and Weidman, S. M. (1959). The relationship between age and the fluoride content of human dentine and enamel. A regional survey. Brit.. Dental J. $107,303-306$.

90. Jenkins, G. N. (1969). Trace elements. Proc. Roy. Soc. Med. 62, 1316-1320.

91. J. J. (1977). Out west, they talked about cadmium. Environ. Sci. and Tech. 11, 336-337.

92. Johansen, E. (1965). Comparison of the ultrastructure and chemical composition of sound and carious enamel from human permanent teeth. Tooth Enamel, M. V. Stack and R. W. Fearnhead, Eds., John Wright and Sons, Ltd., Bristol, U. K., 177-181.

93. Jost, L. (1934). The occurrence of heavy metals in the teeth and salivary glands. Deut. Zahn. Usw. Heilk. 1, 117-122.

94. Kaneko, Y. (1972). The natural occurrence of trace metals in human teeth. II. Estimation of trace metals ( $\mathrm{Pb}, \mathrm{Cu}, \mathrm{Cd}, \mathrm{Zn}$ ) in human teeth from different geographic areas in Japan. Koku Eisei Gakki Zasshi 22, 79-97.

9.5. Kaneko, Y., Inamori, I., and Nishimura M. (1974). Zinc, lead, copper and cadmium in human teeth from different geographical areas in Japan. Bull. Tokyo Dent. Col1. 15, 233-243.

96. Karig, D. E. and Glassley, W. E. (1970). Dacite and related sediment from the West Mariana Ridge, Philippine Sea. Geol. Soc. Amer. Bull. 81, 2143-2146. 
97. Kaushansky, L. I. (1932). Chemical analyses of teeth, roots and crowns affected by pyorrhea alveolaris and dental caries. II. Analyses of phosphorus and magnesium content. Dental Cosmos 74, 468-473.

98. Kelly, J. J. (1972). Neutron-activation analysis. Ultrapurity, M. Zief and R. Speights, Eds., Marcel Dekker, Inc., New York, 535-556.

99. Kubota, J., Lazär, V. A., Losee, F. L., and Curzon, M. E. J. (1974). Determination of strontium in ground and whole teeth by $\mathrm{X}$-ray emission spectrography. J. Dent. Res. 53, 1276-1279.

100. Kuroda, T. (1967). Distribution in human body of naturally occurring radionuclides belonging to the uranium decay series. II. Po-210 and Ra-226 contents in the teeth of the residents in an area with relatively high background of radioactivity, Igaku to Seibutsuzaku, $75,230-233$.

101. Kurosaki, N. and Fusayama T. (1973). Penetration of elements from amalgam into dentin. J. Dent. Res. 52, 309-317.

102. La Fleur, P. D. (Ed.) (1976). Accuracy in trace analysis. Sampling, sample handling, analysis. Proceedings of the $7 \mathrm{th}$ Materials Research Symposium. NBS Special Publication 422.

103. Laitinen, H. A. (1967). Trace characterization by electrochemical methods. Trace characterization, chemical and physical. W. W. Meinke and B. F. Scribner, Eds. NBS Monograph 100, 75-120.

104. Langmyhr, F. J., Sundli, A., and Jonsen, J. (1974). Atomic absorption spectrometric determination of cadmium and lead in dental material by atomization directly from the solid state. Anal. Chim. Acta 73, 81-85.

105. Langmyhr, F. J., Lind, T., and Jonsen, J. (1975). Atomic absorption spectrometric determination of manganese, silver and zinc in dental material by atomization directly from the solid state. Anal. Chim. Acta 80, 297-301.

106. Lanzola, E. and Allegrini, M. (1973). Metabolism of lead in man through measurement of lead-210 as a natural tracer. Proc. Int. Symp. Environ. Health Aspects Lead 1972, 365-371.

107. Lazarev, R. P. (1962). The zinc content of teeth in health and in dental caries. Stomatologiya $41,27-29$.

108: Lerch, P., Geering, J. J., and Lorenz, C. H. (1970). Sr-90 contamination of the skeleton and milk teeth in Switzerland. Symposium International de Radioecologie 2. 1025-1038.

109. Levine, R. S. (1972). Distribution of fluoride in active and arrested carious lesions in dentine. J. Dent. Res. 51, 1025-1029.

110. Litman, R., Finston, H. L., and williams, E. T. (1975). Evaluation of sample pretreatments for mercury determination. Anal. Chem. 47, 2364-2369.

111. Little, M. F. and Steadman, L. T. (1966). Chemical and physical properties of altered and sound enamel-IV. Trace element composition. Arch. Oral Biol. 1l, 273-278. 
112. Lockeretz, w. (1975). Lead content of deciduous teeth of children in different environments. Arch. Environ. Health 30, 583-587.

113. Losee, F. L. and Ludwig, T. G. (1970). Trace elements and caries. J. Dent. Res. 49. 12.29-1.235.

114. Losee, F. L., Little-Mc Clellan, M. F., and Orbell, G. M. (1971). Strontium content of teeth as related to geologic environment. Program and abstracts of papers. International Association for Dental Research, 49th General Session, Abstract 638, 212 .

115. Losee, F. and Little, M. F. (1973). Boron content of human teeth. Program and abstracts of papers. International Association for Dental Research, 5lst General Session, Abstract 273, 127.

116. Losee, F. L., Curzon, M. E. J., and Little, M. F. (1974a). Trace element concentrations in human enamel. Arch. Oral Biol. 19, 467-470.

117. Losee, F. L., Cutress, T. W., and Brown, R. (1974b). Natural elements of the periodic table in human dental enamel. Caries Res. 8, 123-134.

118. Losee, F. L., Cutress, T. W., and Brown, R. (1974c). Trace elements in human dental enamel. Trace substances in environmental health. VIJ. D. D. Hemphill, Ed., Univ. of Missouri, Columbia, Missouri, 13-17.

119. Lovaas, A. I. and Hursh, J. R. (1968). Radium-226 and Lead-210 in human teeth and bones. Health Phys: 14, 549-555.

120. Lundberg, M., Söremark, R., and Thilander, H. (1965a). The concentration of some elements in the enamel of unerupted (impacted) human teeth. Odont. Revy 16, 8-11.

121. Lundberg, M., Söremark, R., and Thilander, H. (1965b). Gamma-ray spectrometric analysis of some elements in coronal dentine of unerupted. (impacted) human teeth. Odont. Revy 16, 97-100.

122. Lundstrom, H. M. (1931). Age changes in the inorganic constituents of sound human teeth. Am. J. Med. Sci. 182, 152.

123. Malik, S. R. and Fremlin, J. H. (1974). A study of lead distribution in human teeth, using charged particle activation analysis. Caries Res. 8 , 283-292.

124. Mamedova, F. M. (1965). Se content in hard human dental tissues under normal conditions, in deep caries, and in periodontopathy. Stomatologiya $\underline{44}, 3-6$.

125. Mamedov, O. R. and Anifaev, T. M. (1974). Effect of iron ore dust on the trace element composition of teeth. Azerb. Med. Zh. 51, 73-75.

126. Manly, R. S. and Hodge, H. C. (1939). Density and refractive index studies of dental hard tissues. J. Dent. Res. 18, 133-141.

127. Marei, A. N., Knizhnikov, V. A. and Yartsev, E. I. (1965). Use of extracted teeth as a method of mass control of Sr-90 content in men. U. S. Atomic Energy Commission, NP-16257. 
128. Marei, A. N. and Borisov, B. K. (1972). Strontium-90 dynamics in the bone tissue of Soviet citizens during 1969-1971. Gig. Sanit. 37, 56-59.

129. Maulbetsch, A. and Rutishauser, E. (1936). Lead in the teeth. Arch. Intern. Pharmacodynamie 53, 55-64.

130. Mc Clure, F. J. and Likins, R. C. (1951). Fluorine in human teeth studied in relation to fluorine in the drinking water. J. Dent. Res. 30, $172-176$.

131.. Miller, Jr., R. G. (1966). Simultaneous statistical inference. McGrawHill Book Company, New York.

132. Minczewski, J. (1967). Preconcentration in trace analysis. Trace characterization, chemical and physical, W. W. Meinke and B. F. Scribner, Eds., NBS Monograph 100, 385-416.

133. Miyazaki, K. (1958). Nickel ions in hard tissues of the human tooth. Kyushu Shika Gakkai Zasshi 10, 64-68.

134. Morrison, D. F. (1976). Multivariate statistical methods, 2nd edition, McGraw-Hill Book Company, New York.

135. Murray, M. M., Glock, G. F., and Lowater, F. (1939). Chemical and spectrographic determination of iron in tooth material. Brit. Dental $\mathrm{J}$. $\underline{66}, 345-350$.

136. Needleman, H. L., Tuncay, O. C., and Shapiro, I. M. (1972). Lead levels in deciduous teeth of urban and suburban American children. Nature 235, $111-112$.

137. Needleman, H. L., and Shapiro, I. M. (1973). Lead in deciduous teeth. A marker of exposure in heretofore asymptomatic children. Proc. Int. Symp. Environ. Health Aspects Lead 1972, 773-778.

138. Needleman, H. L., Davidson, I., Sewell, E. M., and Shapiro, I. M. (1974a). Subclinical lead exposure in Philadelphia school children. Identification by dentine lead analysis. N. Engl. J. Med. 290, 245-248.

139. Needleman, H. L. and Shapiro, I. M. (1974b). Dentine lead levels in asymptomatic Philadelphia school children. Subclinical exposure in high and low risk groups. Environ. Health Perspect. 7, 27-31.

140. Nezhivenko, L. N. (1961a). The Cu content in normal and alveolar pyorrhea-affected teeth. Stomatologiya $\underline{40}, 32-35$.

141. Nezhivenko, L. N. (1961b). Content of $\mathrm{Cu}$ in teeth in caries and diabetes mellitus. Stomatologiya $40,24-26$.

142. Nezhivenko, L. N., Storozheva, N. N., Vikhm, N. A., Mamedova, F. M., and Borisova, N. N. (1967). Content of certain trace elements in hard tooth tissues in parodontopathy. Parodontoz, 155-160.

143. Nixon, G. S. and Smith, H. (1960). Estimation of arsenic in teeth by activation analysis. J. Dent. Res. 39, 514-516. 
144. Nixon, G. S: and Smith, H. (1962). Estimation of copper in human enamel by activation analysis. J. Dent. Res. 41, 1013-1016.

145. Nixon, G. S., Paxton, G. D., and Smith, H. (1965). Estimation of mercury in human enamel by activation analysis. J. Dent. Res. 44, 654-656.

146. Nixon, G. S., Livingston, H. D., and Smith, H. (1966). Estimation of manganese in human enamel by activation analysis. Arch. Oral Biol. 11, 247-252.

147. Nixon, G. S., Livingston, H. D., and Smith, H. (1967a). Estimation of antimony in human enamel by activation analysis. Caries Res. 1, 327-332.

148. Nixon, G. S., Livingston, H. D., and Smith, H. (1967b). Estimation of zinc in human enamel by activation analysis. Arch. Oral Biol. 12, 411-416.

149. Nixon, G. S., Smith, H., and Livingston, H. D. (1967c). Trace elements in human tooth enamel. Nucl. Activ. Tech. Life Sci., Proc. Symp., Amsterdam, 455-462.

150. Nixon, G. S. (1969). Trace element content of the hard dental tissues and dental plaque. Caries Res. 3 , 60-74.

151. Nixon, G. S. and Myers, V. B. (1970). Estimation of selenium in human dental enamel by activation analysis. Caries Res. 4 , 179-187.

152. Ohira, M., Yoshioka, S., Masuda, K., and Matsumoto, S. (1973). Radium-226, lead-210, and polonium-210 in food, water, and human teeth. Nippon Eiseigaku Zasshi 27, 544-550.

153. Olshen, R. A. (1977). Comment. J. Amer. Statist. Assoc. 72, 144-146.

154. Otani, K. (1957). Studies on the absorption and distribution of arsenic. Sapporo Igaku Zasshi 11, 285-294.

155. Paulhamus, J. A. (1972). Airborne contamination. Ultrapurity, M. Zief and R. Speights, Eds., Marcel Dekker, Inc., New York, 255-292.

156. Pearlman, R. S., Hefferren, J. J., and Lyon, H. W. (1970). Determination of tin in enamel and dentin by atomic absorption spectroscopy. J. Dent. Res. 49, 1437-1443.

157. Petrow, H. G. and Cover, A. (1965). Direct radiochemical determination of lead-210 in bone. Anal. Chem. 37, 1659-1660.

158. Pfrieme, F. (1934). Normal and pathological lead contents of teeth in man and animals. Arch. Hyg. Bakt. 111, 232-242.

159. Pieruccini, R. and zotti, R. (1949). Biological function of strontium and manganese in teeth. Atti Soc. Toscana Sci. Nat. (Pisa), Mem. Ser. A $\underline{56}, 119-126$.

160. Prokofiev, O. N., Antonova, V. A., Dikaya, F. Y., Dzyubenko, I. I., and Khazina, M. A. (1973).! Strontium-90 in human bone tissue and teeth. Mater. Vses. Simp. Teor. Prakt. Aspekty Deistviya Malykh Doz Ioniz. Radiats. 158-160. 
161. Rasmussen, E. G. (1974). Antimony, arsenic, bromine and mercury in enamel from human teeth. Scand. J. Dent. Res. 82, 562-565.

162. Retief, D. H., Van Wyk, C. W., and Turkstra, J. (1969). Qualitative and comparative study of elements in teeth by neutron activation and high resolution gamma spectrometry. J. S. Afr. Dent. Assoc. 24, 75-80.

163. Retief, D. H., Cleaton-Jones, P. E., and Turkstra, J. (1970a). The quantitative determination of $\mathrm{Ca}, \mathrm{Na}, \mathrm{Al}, \mathrm{Mg}$, and $\mathrm{Cl}$ in normal enamel and dentine by neutron activation and high resolution gamma spectrometry. J. S. Afr. Dent. Assoc. 25, 188-192.

164. Retief, D. H., Cleaton-Jones, P. E., Turkstra, J., and De Wet, W. J. $(1970 \mathrm{~b})$. The quantitative analysis of $\mathrm{Cr}, \mathrm{Ba}, \mathrm{Sb}, \mathrm{Ag}, \mathrm{Zn}, \mathrm{Co}$, and $\mathrm{Fe}$ in normal human enamel and dentine by neutron activation and high resolution gamma-spectrometry. J. S. Afr. Dent. Assoc. 25, 370-375.

165. Retief, D. H., Cleaton-Jones, P. E., Turkstra, J., and De Wet, W. J. (1971a). The quantitative analysis of sixteen elements in normal human enamel and dentine by neutron activation analysis and high-resolution gamma-spectrometry. Arch. Oral Biol. 16, 1257-1267.

166. Retief, D. H., Cleaton-Jones, P. E., Turkstra, J., and De Wet, W. J. (1971b). The quantitative analysïs of $\mathrm{Sr}, \mathrm{Au}, \mathrm{Br}, \mathrm{Mn}$ and $\mathrm{Na}$ in normal human enamel and dentine by neutron activation and high resolution gamma spectrometry. J. S. Afr. Dent. Assoc. 26, 63-69.

167. Retief, D. H., Scanes, S., Cleaton-Jones, P. E., Turkstra, J., and Smit, H. J. (1974). The quantitative analysis of selenium in sound human enamel by neutron activation analysis. Arch. Oral. Biol. 19, 517-523.

168. Robertson, D. E. (1972). Contamination problems in trace-element analysis and ultrapurification. Ultrapurity, M. Zief and R. Speights, Eds., Marcel Dekker, Inc., New York, 207-254.

169. Rowland, R. E. (1963). Radium in human teeth. A quantitative autoradiographic study. Arch. Oral Biol. 8, 13-21.

170. Rowles, S. L. (1967). Chemistry of the mineral phase of dentine. Structural and chemical organization of teeth, Vol. 2, A. E. W. Miles, Ed., Academic Press, New York, 201-246.

17.1. Rubin, D. B. (1976). Noniterative least squares estimates, standard errors and F-Tests for analysis of variance with missing data. J.R. Statist. Soc. B 38, 270-274.

172. Rytomaa I. (1971). Strontium-90 in deciduous teeth collected in Helsinki from chilidren born in 1956-1963. Acta Odontol. Scand. 29, 327-336.

173. Rytomaa, I. and Tuompo, H. (1974). Lead levels in deciduous teeth. Naturwissenshaften 61,363 . 
174. Sairenji, E., Urata, Y., Yamada, M., Suzuki, T., and Sasaki, E. (1961). Neutron activation analysis of arsenic in the dental hard tissues of arsenic-devitalized teeth. J. Nippon Univ. School Dentistry 4, 75-80.

175. Samsahl, K. and söremark, R. (1961a). Comparative and absolute measurements of 11 inorganic constituents of 38 human tooth samples with gammaray spectrometry. Proc. Intern. Conf. Modern Trends in Activation Analysis, $149-154$.

176. Samsahl, K. and söremark, R. (1961b). Comparative and absolute measurements of 11 inorganic constituents of 38 human tooth samples with gammaray spectrometry. Aktiebolaget Atomenergi, Stockholm.

177. Scheffe, H. (1959). The analysis of variance. J. Wiley and Sons, Inc., New York.

178. Scheffe, H. (1977a). A note on a reformulation of the S-method of multiple comparison. J. Amer. Statist. Assoc. 72, 143-144.

179. Scheffe, H. (1977b). Rejoinder: J. Amer. Statist. Assoc. $72,146$.

180. Serebryakova, N. N. (1963). Manganese content of teeth of normal subjects and patients with caries or parodontitis. Stomatologiya $42,40-44$.

181. Shapiro, I. M., Needleman, H. L., and Tuncay, O. C. (1972). The lead content of human deciduous and permanent teeth. Environ. Res. 5, 467-470.

182. Shapiro, I. M., Dobkin, B., Tuncay, O. C., and Needleman, H. L. (1973). Lead levels in dentine and circumpulpal dentine of deciduous teeth of normal and lead poisoned children. Clin. Chim. Acta 46, 119-123.

183. Shapiro, I. M., Mitchell, G., Davidson, I., and Katz, S. H. (1975). The lead content of teeth. Evidence establishing new minimal levels of exposure in a living preindustrialized human population. Arch. Environ. Health $30,483-486$.

184. Shaw, J. H., Resnick, J. B., and Sweeney, E. A. (1959). Fluoride content of human teeth from the orient and the Canadian Arctic. J. Dent. Res. 38 , 129-134.

185. Shimada, K. (1971). Manganese concentration in the teeth of Japanese. Shikoku Igaku Zasshi (27), 516-526.

186. Smales, A: A. (1967). Radioactivity techniques in trace characterization. Trace characterization, chemical and physical, W. W. Meinke and B. F. Scribner, Eds., NBS Monograph 100, 307-336.

187. Snooks, E. C. (1972). Emission spectroscopy. Ultrapurity, M. Zief and R. Speights, Eds., Marcel Dekker, Inc., New York, 437-475.

188. Söremark, R. and Samsahl, K. (1962a). Gamma-ray spectrometric analysis of elements in normal human enamel. Arch. Oral Biol. 6 , 275-283. 
189. Söremark, R. and Samsahl, K. (1962b). Gamma-ray spectrometric analysis of elements in. normal human dentin. J. Dent. Res. 41, 603-606.

190. Söremark, R. and Lundberg, M. (1964a). Gamma-ray spectrometric analysis of the concentrations of $\mathrm{Cr}, \mathrm{Ag}, \mathrm{Fe}, \mathrm{Co}, \mathrm{Rb}$, and $\mathrm{Pt}$ in normal human enamel. Acta Odontol. Scand. 22, 255-259.

191. Söremark, R. and Lundberg, M. (1964b). Analysis of the concentrations of $\mathrm{Cr}, \mathrm{Ag}, \mathrm{Fe}, \mathrm{Co}, \mathrm{Pt}$, and $\mathrm{Rb}$ in normal human dentine. Odont. Revy 15 , 285-289.

192. Söremark, R., Wing, K., Olsson, K., and Goldin, J. (1968). Penetration of metallic ions from restorations into teeth. J. Pros. Dent. 20, 531-540.

193. Steadman, L. T., Hodge, H. C., and Horn, H. W. (1941). Spectrochemical studies of potassium in bone and tooth substance. J. Biol. Chem. 140, $71-76$.

194. Steadman, L. T., Brudevold, F., and Smith, F. A. (1958). Distribution of strontium in teeth from different geographic areas. J. Amer. Dent. Assoc. 57, 340-344.

195. Steadman, L. T., Brudevold, F., Smith, F. A., Gardner, D. E., and Little, M. F. (1959). Trace elements in ancient teeth. J. Dent. Res. 38, 285-292.

196. Steinnes, E., Dahm, S., and Furseth R. (1974). Concentration of rare earths in dentine and enamel. A pilot study. Acta Odontol. Scand. 32, 125-129.

197. Stewart, D. J. (1974). Teeth as indicators of exposure of children to lead. Arch. Dis. Child. 49, 895-897.

198. Storozheva, N. N. (1963). Content of $\mathrm{Pb}$ and $\mathrm{Sn}$ in healthy and carious teeth. Stomatologiya $42,44-48$.

199. Strehlow, C. D., and Kneip, T. J. (1969). The distribution of lead and zinc in the human skeleton. Amer. Ind. Hyg. Ass. J. 30, 372-378.

200. Strehlow, C. (1972). The use of deciduous teeth as indicators of lead exposure. Ph.D. Thesis, New York University.

201. Stubbins, M., and Fremlin, J. H. (1967). Distribution of carbon, calcium and phosphorus in dental enamel studied by charged-particle activation analysis. Nucl. Activ. Tech. Life Sci., Proc. Symp., Amsterdam, 463-478.

202. Suchkov, B. P., Katsap, I.M., and Gulgasenko, A. I. (1973). Caries incidence among the population of the Chernovitsi region in relation to the selenium content of the teeth. Stomatologiya $22,21-23$. 
203. Sukhatme, P. V., and Sukhatme, B. V. (1970). Sampling theory of surveys with applications. Iowa State University Press, Ames, Iowa.

204. Tefft, H., French, E. L., :and Hodge, H. C. $\because(1941) !$ Mägnësium determinations on all the dentine from sound and carious teeth. J. Dent. Res. $20,45-48$.

205. Thompson, G., and Bankston, D. C. (1970). Sample contamination from grinding and sieving determined by emission spectrometry. Applied Spectroscopy 24, 210-219.

206. Tölg, G. (1972). Extreme trace analysis of the elements--I. Methods and problems of sample treatment, separation and enrichment. Talanta $19,1489-1521$.

207. Van Wyk, C. w.., and Retief;, D: H. (1968). Detection of elements in teeth by means of neutron activation. Diastema 2 ; 33-35.

208. Vihm, N. (1964). Nickel and silver content in intact human teeth, in caries, and in alveolar pyorrhea. Uch. Zap. Tartusk, Gos. Univ. 163. 515-520.

209. Vikhm, N. A. (1962): Content of $\mathrm{Ni}$ and Ag in healthy teeth, dental caries, and alveolar pyorrhea. Stomatologiya 41, 13-17.

210. Vikhm, N. A. (1963). Content of arsenic in the teeth in health, dental caries, and alveolar pyorrhea. Stomatologiya 42, 23-25.

211. Wainwright, W. W., Butt, B. G., Hauptfuehrer, J. D., and Dute, H. L. (1953). Deposition of radioactive iodine in teeth of persons treated for disease of the thyroịd gland. J. Amer. Dent. Assoc. 47, 649-655.

212. Washio, M. (1971). Penetration of silver agents into dentine. 'Shika Igaku $34,733-746$.

213. Weatherell, J. A., Robinson, C., and Hallsworth, A. S. (1974). Variations in the chemical composition of human enamel. J. Dent. Res. 53, 180-191.

214. Weiss, R. H. (1972). Visible spectrophotometry. Ultrapurity, M. Zief and R. Speights, Eds., Marcel Dekker, Inc., New York, 557-574.

215. Wei, S. H., and Forbes, W. C. (1974). Electron microprobe investigations of stannous fluoride reactions with enamel surfaces. J. Dent: Res. 53, 51-56.

216. West, T. S. (1967). Spectrophotometry in trace characterization. Trace characterization, chemical and physical, w. W. Meinke and B. F. Scribner, Eds., NBS Monograph 100, 215-302.

217. Wyss, V. (1951). Spectrographic determination of lead in the teeth of normal individuals and of individuals exposed to lead poisoning. Rass. Med. Ind. 20, 40-47. 
218. Yoon, S. H., Brudevold, F., Gardner, D. E., and Smith, F. A. (1960). Distribution of fluoride in teeth from areas with different levels of fluoride in the water supply. J. Dent. Res. 39, 845-856.

219. Zahradnik, R. T. and Moreno, E. C. (1975). Structural features of human dental enamel as revealed by isothermal water vapour sorption. Arch. Oral Biol. 20, 317-325.

220. Zakson, M. L. (1968). Trace elements in teeth of elderly and senile persons. Dopov. Akad. Nauk. Ukr. Rsr, Ser. B, 30, 812-813.

221. Zief, M. and Nesher, A. G. (1974). Clean environment for ultratrace analysis. Environ. Sci. and Tech. 8, 677-678.

222. Zipkin, I. (1970). The inorganic composition of bones and teeth. Biol. Calcification, Cell. and Mol. Aspects, H. Schraer, ed. 69-103. 


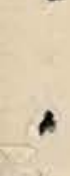

- 